Aus der Klinik für Anästhesiologie

(Prof. Dr. med. M. Quintel)

der Medizinischen Fakultät der Universität Göttingen

\title{
Prospektive Untersuchung zur Wirksamkeit wärmeprotektiver Verfahren unter präklinischen Bedingungen
}

\section{Eine Probandensimulation}

\author{
INAUGURAL- DISSERTATION \\ zur Erlangung des Doktorgrades \\ der Medizinischen Fakultät der \\ Georg-August-Universität zu Göttingen \\ vorgelegt von \\ Annette Lorey-Tews \\ aus Hamburg
}

Göttingen 2017 
Dekan:

Referent:

Ko-Referent/in:

Drittreferent/in:

Datum der mündlichen Prüfung: $\quad$ 03.05.2018
Prof. Dr. rer. nat. H. K. Kroemer

Prof. Dr. med. A. Bräuer

Prof. Dr. med. Klaus Dresing

Prof. Dr. mult. Thomas Meyer 
Hiermit erkläre ich, die Dissertation mit dem Titel "Prospektive Untersuchung zur Wirksamkeit wärmeprotektiver Verfahren unter präklinischen Bedingungen - Eine Probandensimulation" eigenständig angefertigt und keine anderen als die von mir angegebenen Quellen und Hilfsmittel verwendet zu haben.

Göttingen, den

(Unterschrift) 


\section{Inhaltsverzeichnis}

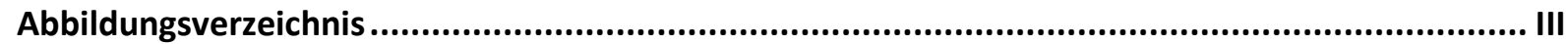

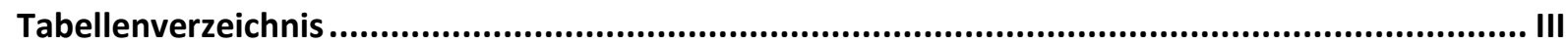

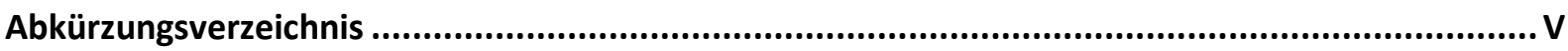

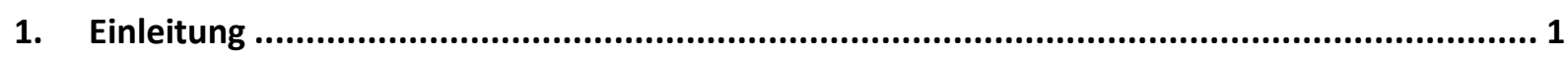

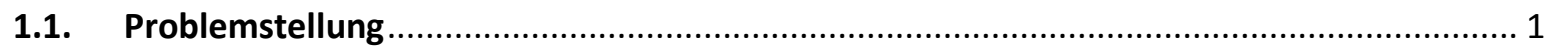

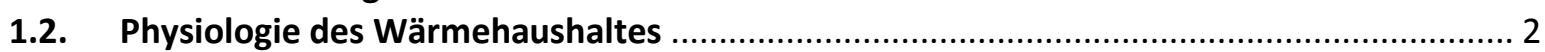

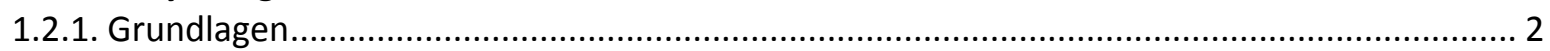

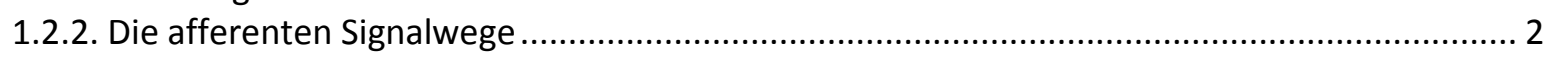

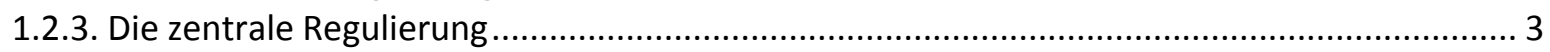

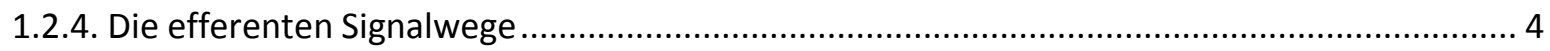

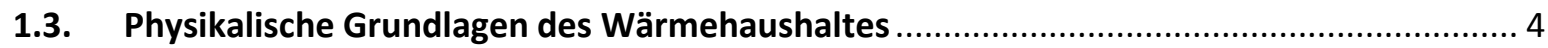

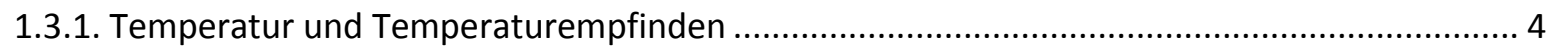

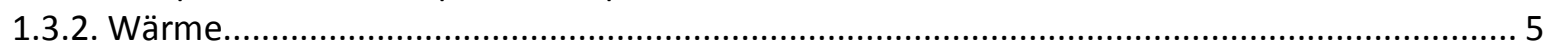

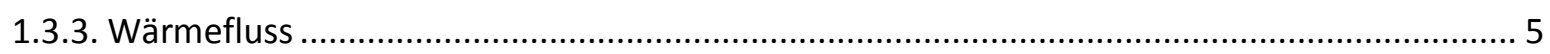

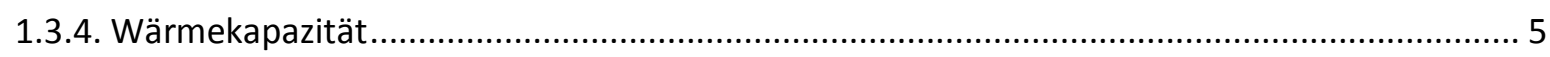

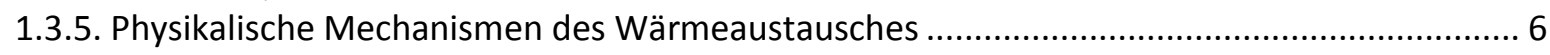

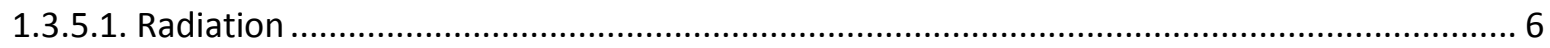

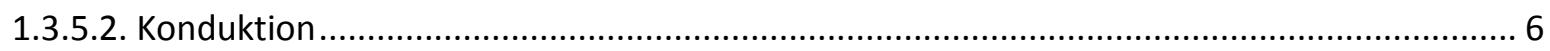

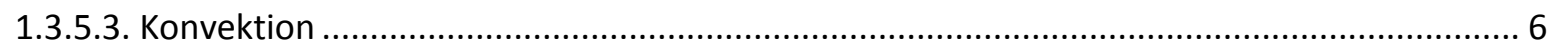

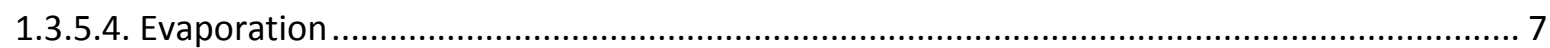

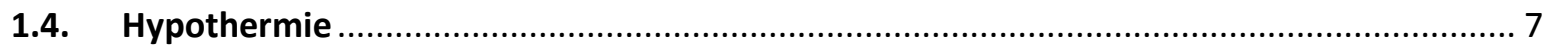

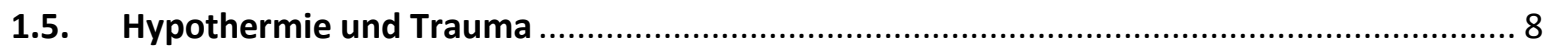

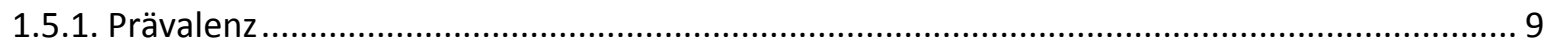

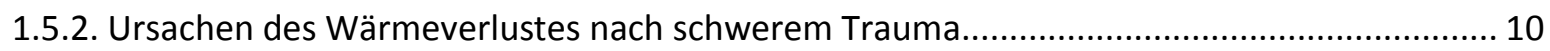

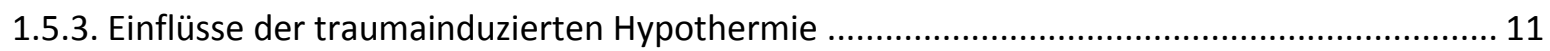

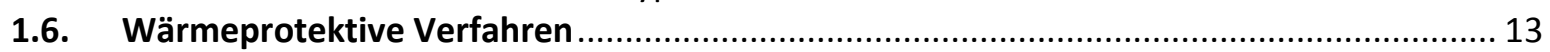

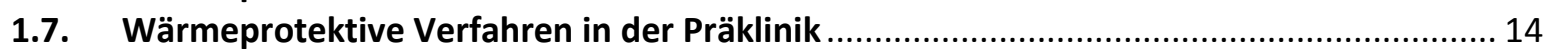

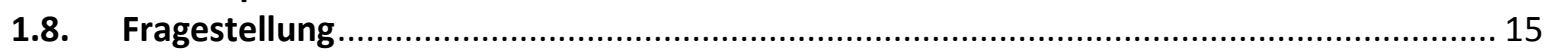

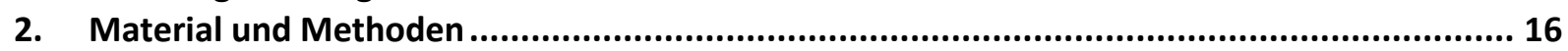

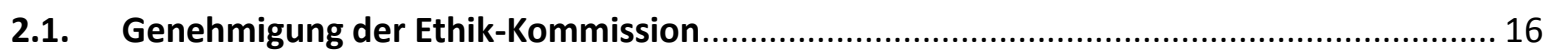

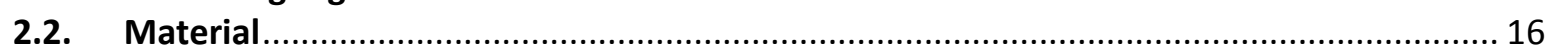

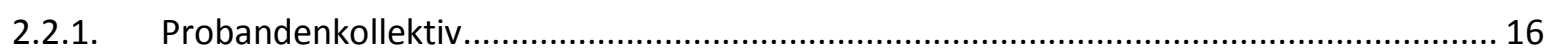

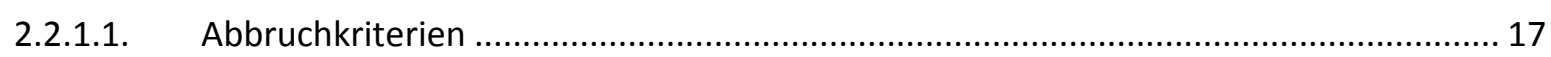

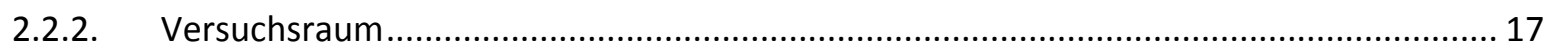

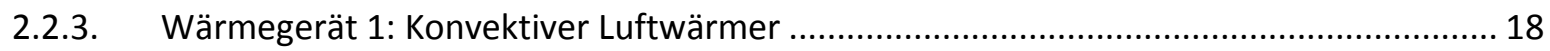

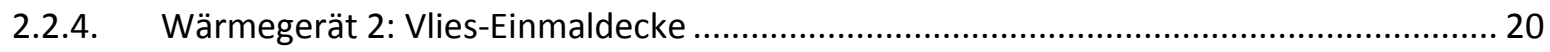

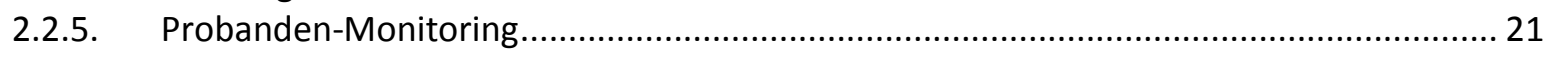

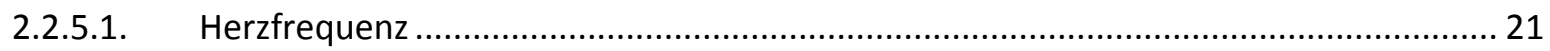

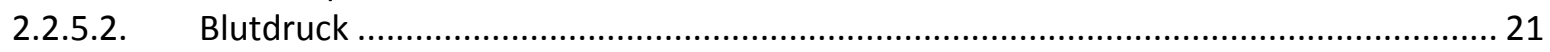

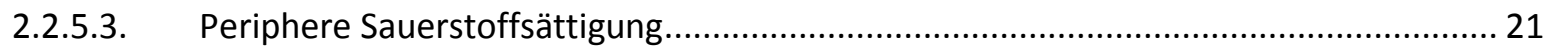

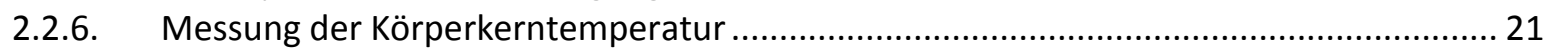

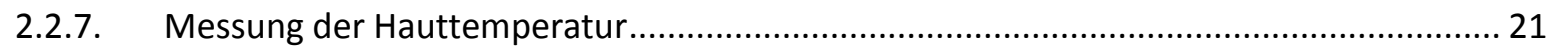

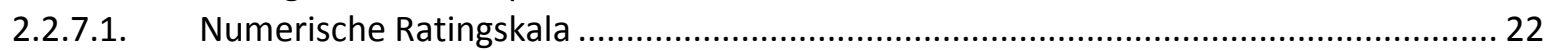

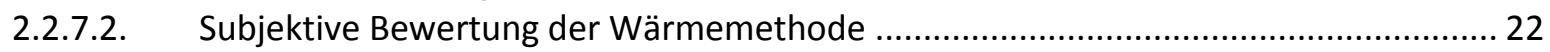

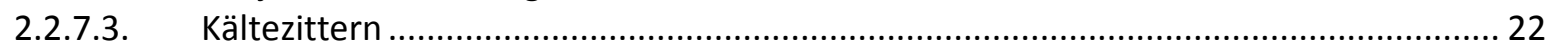

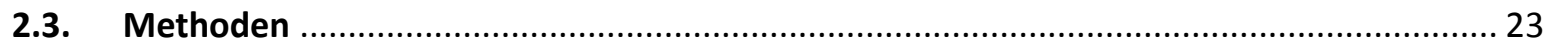

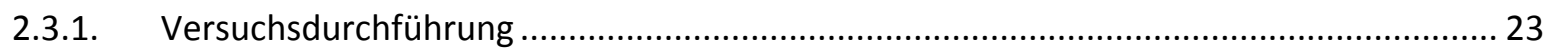

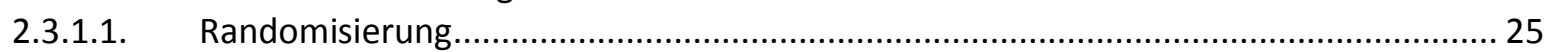




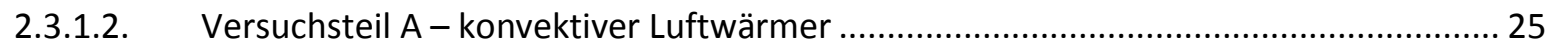

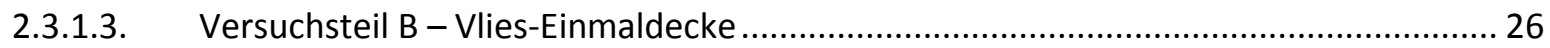

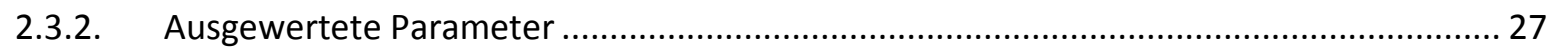

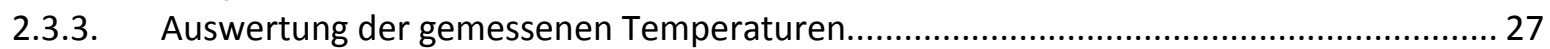

2.3.3.1. Mittlere Hauttemperatur nach Ramanathan ............................................................ 27

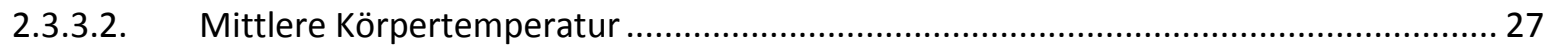

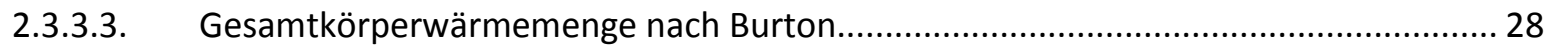

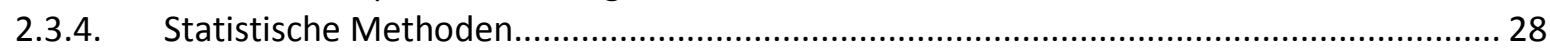

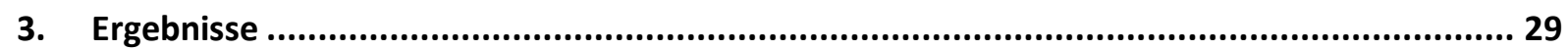

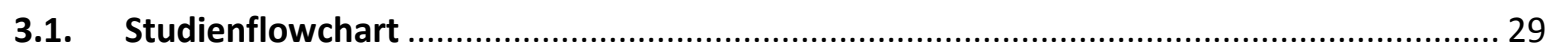

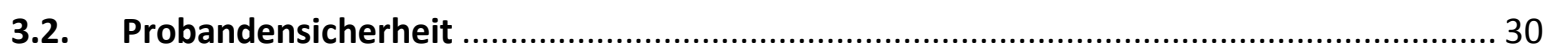

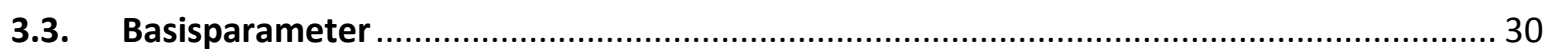

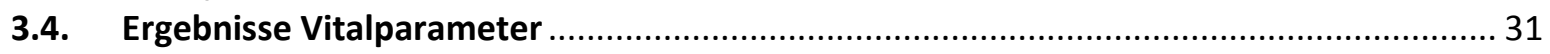

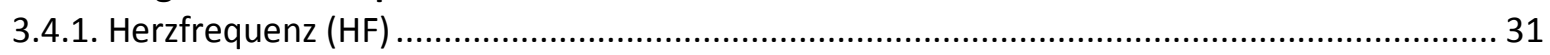

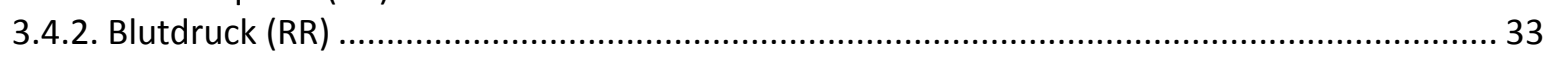

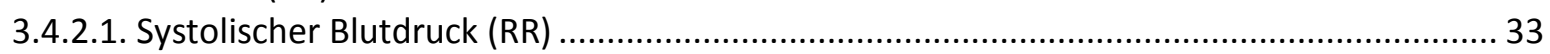

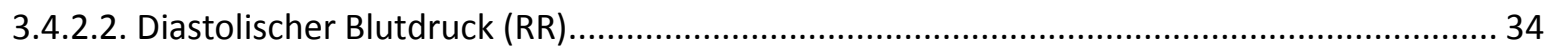

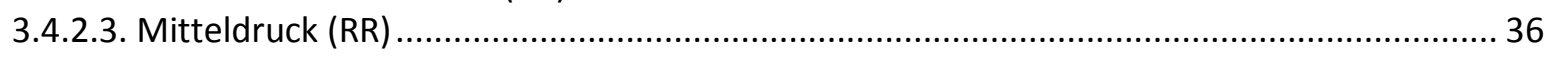

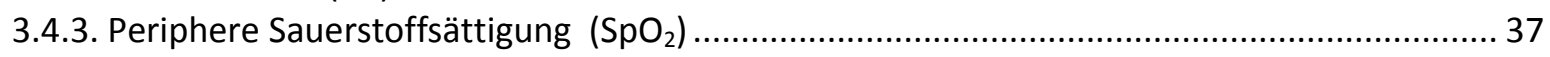

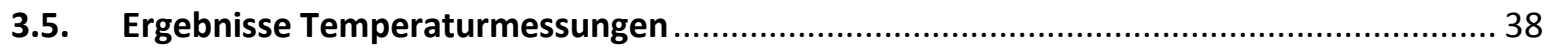

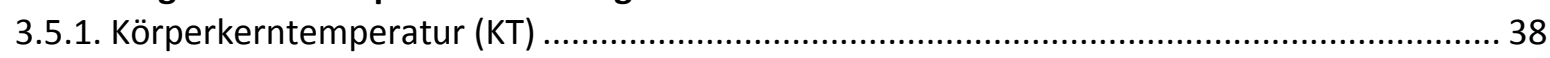

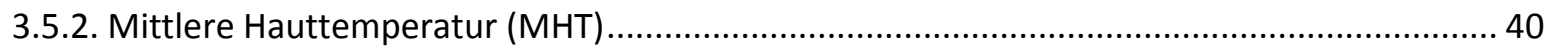

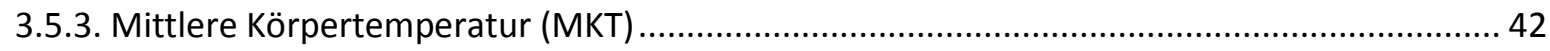

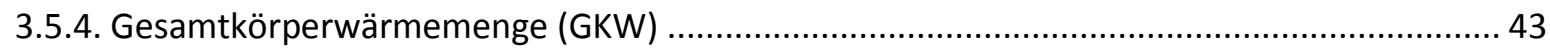

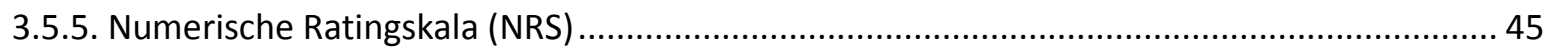

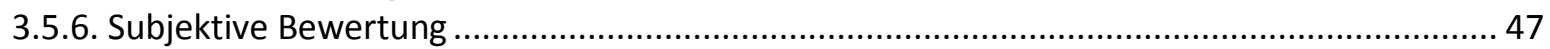

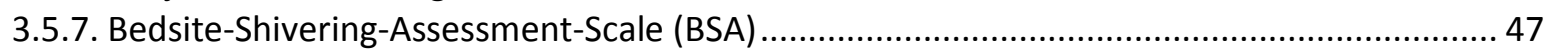

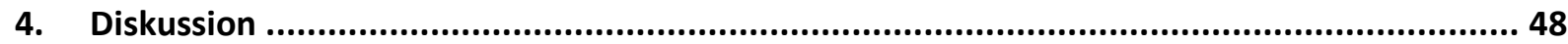

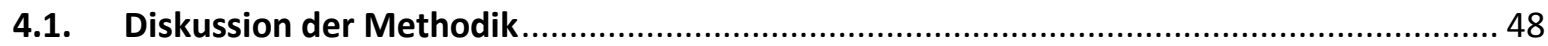

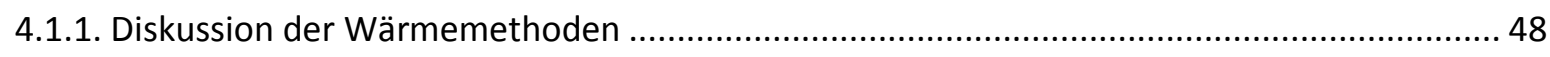

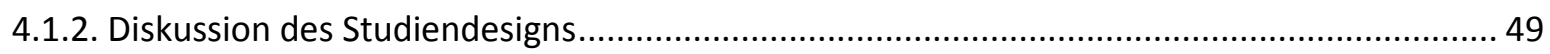

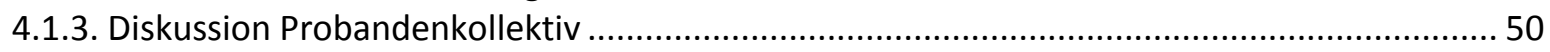

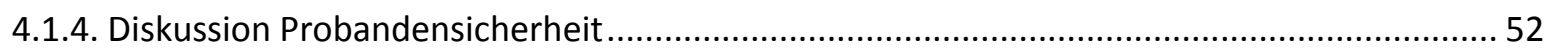

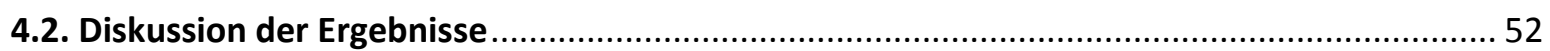

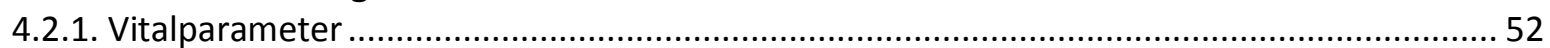

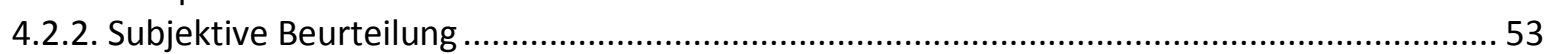

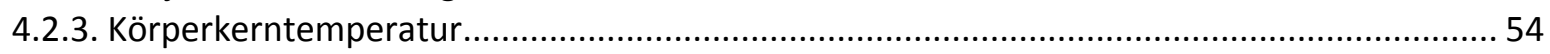

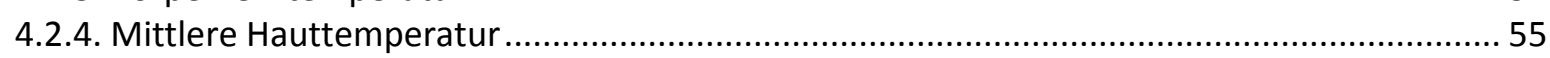

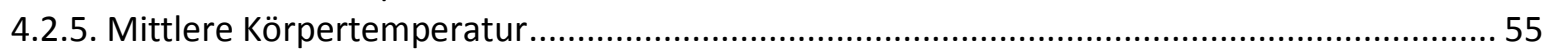

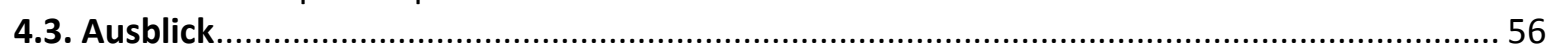

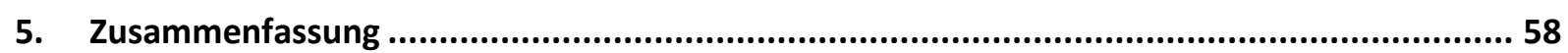

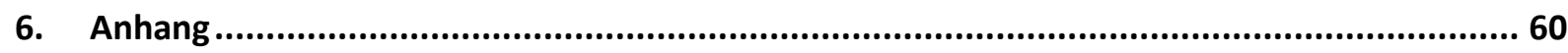

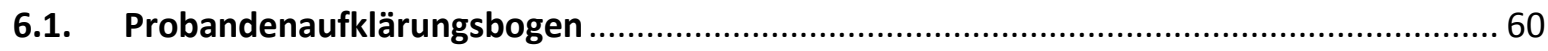

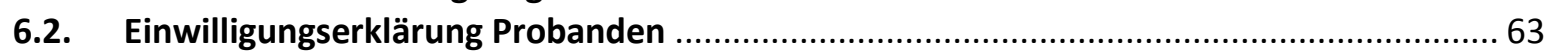

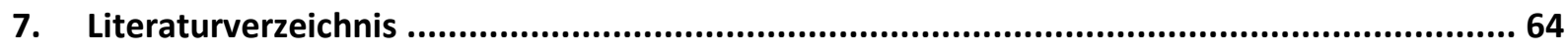

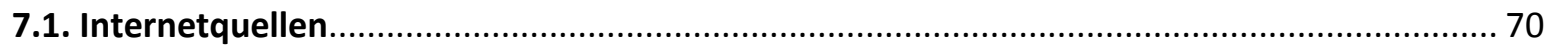




\section{Abbildungsverzeichnis}

Abbildung 1: Regelkreis der Thermoregulation (A. Lorey-Tews)

Abbildung 2: konvektiver Luftwärmer Warmtouch WT 5900 der Firma Nellcor. Quelle: A. Lorey-Tews

Abbildung 3: Bedienfeld des Warmtouch WT 5900 der Firma Nellcor. Quelle: A. Lorey-Tews............. 19

Abbildung 4: Vlies-Einmaldecke zusammengelegt. Quelle: A. Lorey-Tews.......................................... 20

Abbildung 5: Darstellung der Füllung der Vlies-Einmaldecke. Quelle A. Lorey-Tew............................ 20

Abbildung 6: Numerische Ratingskala. Quelle: A. Lorey-Tews......................................................... 22

Abbildung 7: Überblick allgemeiner Versuchsaufbau. Quelle: A. Lorey-Tews ..................................... 24

Abbildung 8: Versuchsteil A - konvektiver Luftwärmer. Quelle: A. Lorey-Tews ................................... 25

Abbildung 9: Versuchsaufbau B - Vlies-Einmaldecke. Quelle: A. Lorey-Tews ...................................... 26

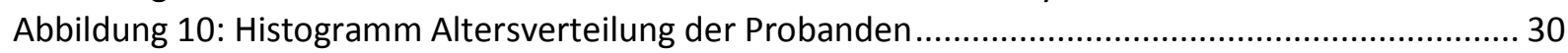

Abbildung 11: Herzfrequenz im Versuchsteil mit konvektiver Luftwärmung ..................................... 32

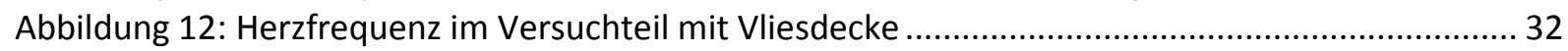

Abbildung 13: Systolischer Blutdruck im Versuchsteil mit konvektiver Luftwärmung .......................... 33

Abbildung 14: Systolischer Blutdruck im Versuchteil mit Vliesdecke ................................................ 34

Abbildung 15: Diastolischer Blutdruck im Versuchsteil mit konvektiver Luftwärmung ........................ 35

Abbildung 16: Diastolischer Blutdruck im Versuchteil mit Vliesdecke................................................. 35

Abbildung 17: Mitteldruck im Versuchsteil mit konvektiver Luftwärmung ......................................... 36

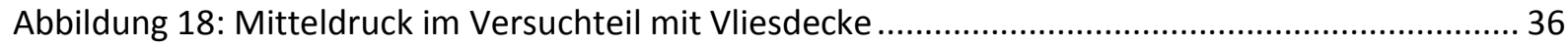

Abbildung 19: Periphere Sauerstoffsättigung im Versuchsteil mit konvektiver Luftwärmung............. 37

Abbildung 20: Periphere Sauerstoffsättigung im Versuchteil mit Vliesdecke...................................... 38

Abbildung 21: Körperkerntemperatur im Versuchsteil mit konvektiver Luftwärmung ........................ 39

Abbildung 22: Körperkerntemperatur im Versuchteil mit Vliesdecke ................................................. 39

Abbildung 23: Mittlere Hauttemperatur im Versuchsteil mit konvektiver Luftwärmung .................... 41

Abbildung 24: Mittlere Hauttemperatur im Versuchteil mit Vliesdecke ............................................ 41

Abbildung 25: Mittlere Körpertemperatur im Versuchsteil mit konvektiver Luftwärmung .................. 42

Abbildung 26: Mittlere Körpertemperatur im Versuchteil mit Vliesdecke ......................................... 43

Abbildung 27: Gesamtkörperwärmemenge im Versuchsteil mit konvektiver Luftwärmung ................ 44

Abbildung 28: Gesamtkörperwärmemenge im Versuchsteil mit Vliesdecke....................................... 45

Abbildung 29: Numerische Ratingskala im Versuchsteil mit konvektiver Luftwärmung ...................... 46

Abbildung 30: Numerische Ratingskala im Versuchteil mit Vliesdecke .......................................... 46

Abbildung 31: Unfallgeschehen nach Alter und Geschlecht in Deutschland. (Unfallbericht 2013)...... 51

Abbildung 32: Altersverteilung nach Jahren in der vorliegenden Studie. Quelle: A. Lorey-Tews......... 51

\section{Tabellenverzeichnis}

Tabelle 1: Ursachen der Hypothermie ........................................................................................... 7

Tabelle 2: Vergleich der perioperativen wärmeprotektiven Maßnahmen (Bräuer et al. 2006) ........... 13

Tabelle 3: Einschluss- und Ausschlusskriterien Probandenkollektiv ................................................. 16

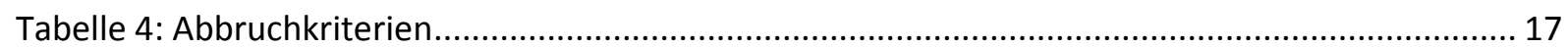

Tabelle 5: Bedsite- Shivering- Assessment- Scale (BSAS) (Badjatia et al. 2008)................................... 23

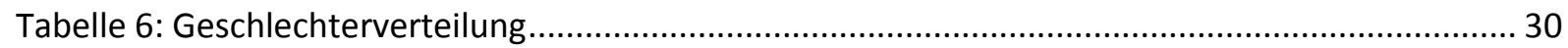

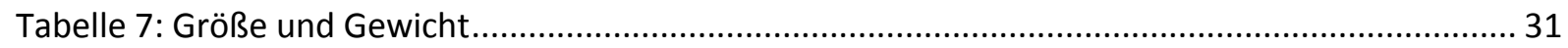

Tabelle 8: Wilcoxon-Test Herzfrequenz Luftwärmer / Vliesdecke ..................................................... 32

Tabelle 9: Wilcoxon-Test systolischer Blutdruck Luftwärmer / Vliesdecke ......................................... 34

Tabelle 10: Wilcoxon-Test diastolischer Blutdruck Luftwärmer / Vliesdecke ...................................... 35

Tabelle 11: Wilcoxon-Test Mitteldruck Luftwärmer / Vliesdecke........................................................ 37

Tabelle 12: Wilcoxon-Test periphere Sauerstoffsättigung Luftwärmer / Vliesdecke ............................ 38

Tabelle 13: Wilcoxon-Test Körperkerntemperatur Luftwärmer / Vliesdecke....................................... 39

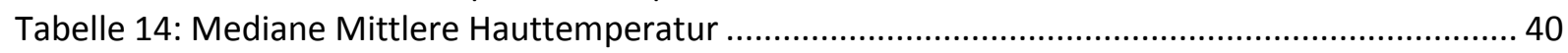

Tabelle 15: Wilcoxon-Test Mittlere Hauttemperatur Luftwärmer / Vliesdecke .................................. 41 


\section{Tabellenverzeichnis}

Tabelle 16: Mediane Mittlere Körpertemperatur

Tabelle 17: Wilcoxon-Test Mittlere Körpertemperatur Luftwärmer / Vliesdecke ................................ 43

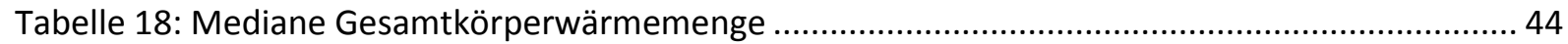

Tabelle 19: Wilcoxon-Test Gesamtkörperwärmemenge Luftwärmer / Vliesdecke .............................. 45

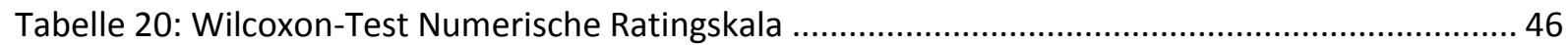

Tabelle 21: Subjektive Bewertung im Versuchsteil mit konvektiver Luftwärmung .............................. 47

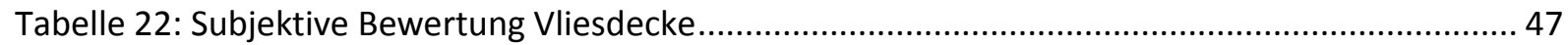




\section{Abkürzungsverzeichnis}

\begin{tabular}{lll} 
AC & - & Wechselstrom \\
ASA & - & American Society of Anesthesiologists \\
ATP & - & Adenosintriphosphat \\
BMI & - & Body-Mass-Index \\
$b p m$ & - & beats per minute \\
BSAS & - & Bedsite-Shivering-Assessment-Scale \\
CDC & - & Center of Desease Control and Prevention \\
DGU & - & Deutsche Gesellschaft für Unfallchirurgie \\
diast. RR & - & Diastolischer Blutdruck \\
GKW & - & Gesamtkörperwärmemenge \\
GP & - & Glycoprotein \\
HF & - & Herzfrequenz \\
ISS & - & Injury-Severety-Score \\
kJ & - & Kilojoule \\
KKT & - & Körperkerntemperatur \\
KOF & - & Körperoberfläche \\
lat. & - & Latein \\
MHT & - & Mittlere Hauttemperatur \\
MKT & - & Mittlere Körpertemperatur \\
mmHg & - & Millimeter Quecksilbersäule \\
MODS & - & Multi-Organ-Dysfunction-Syndrom \\
n & - & Anzahl \\
NRS & - & Numerische Rating-Skala \\
pAVK & - & periphere arterielle Verschlusskrankheit \\
PKW & - & Personenkraftwagen \\
Q & - & Wärmefluss \\
RR & - & Blutdruck (Noninvasiv gemessener Blutdruck) \\
SaO2 & - & arterielle Sauerstoffsättigung \\
SHT & - & Schädel-Hirn-Trauma \\
SpO2 & - & periphere Sauerstoffsättigung \\
V & - & Volumen \\
\hline
\end{tabular}




\section{Einleitung}

\subsection{Problemstellung}

Jedes Jahr erleiden in Deutschland über 16000 Patienten eine so schwere Verletzung, dass sie die Merkmale eines Polytraumas erfüllen. Bei ca. 6.000 ist hierfür die Ursache ein Verkehrsunfall (Jahresbericht Traumaregister 2015). Häufig kann bei diesem Patientenkollektiv als Folge der Verletzungen und der präklinisch begonnenen Maßnahmen zur Stabilisierung der Vitalfunktionen eine Hypothermie beobachtet werden (Aitken et al. 2009, Wade et al. 2011, Trentzsch et al. 2012, Mommsen et al. 2013). Die deletären Auswirkungen der Hypothermie, insbesondere auf die Gerinnung, das Immunsystem sowie das Outcome des Schwerverletzten, sind wissenschaftlich belegt (Shafi et al. 2005, Wang et al. 2005, Crandall und González-Alonso 2010).

Als Erkenntnis dieser Studien wird die frühzeitige Vermeidung von Unterkühlungen und die frühestmögliche Wiedererwärmung gefordert (Lier 2008). Für den innerklinischen Bereich haben sich hierfür aktive Wärmemethoden, insbesondere der Einsatz von konvektiven Luftwärmern, etablieren können und sind durch viele Studien in ihrer Wirksamkeit belegt (Bräuer et al. 2002).

Die Wiedererwärmung erfolgt jedoch auch mit diesen Wärmemethoden nur sehr langsam. Der Focus der Hypothermiebehandlung muss daher auf der Vermeidung und auf dem frühzeitigen Beginn der Behandlung von hypothermen Zuständen liegen (Martin et al. 2005, Hess und Lawson 2006, Hildebrand et al. 2009, Kobbe et al. 2009) .

Für die präklinische Phase, die im Durchschnitt einen Zeitraum von 71 Minuten zwischen Unfallzeitpunkt und Einlieferung in ein Krankenhaus beschreibt (Jahresbericht Traumaregister 2015) bestehen bisher jedoch nur wenige Strategien, einem Wärmeverlust vorzubeugen, geschweige denn zu therapieren. Zumeist kommen ausschließlich passive Wärmemethoden, wie Decken o.ä., zum Einsatz.

Der Einsatz von aktiven Wärmemethoden, wie z.B. konvektiven Luftwärmern am Unfallort, stellt bisher eine Rarität dar. Vorgesehen für den Einsatzbereich im Schockraum oder Operationssaal fehlen bisher Nachweise für die Wirksamkeit in einem deutlich niedrigeren Temperaturumfeld, wie es häufig am Unfallort auftritt, sowie unter präklinischen Bedingungen. Mit dieser Untersuchung soll die Wirksamkeit von konvektiven Luftwärmern, wie sie standardisiert in innerklinischen Bereichen Anwendung finden (Hynson und Sessler 
1992, Bennett et al. 1994, Bock et al. 1998, Bräuer et al. 2000), unter präklinischen Bedingungen überprüft werden.

Ziel dieser Studie ist die Überprüfung der Wirksamkeit dieser Methode, ob diese bereits in der frühen Phase der Traumaversorgung wirksam ist und ob die beschriebenen Folgen der Hypothermie vermieden oder gemindert werden können.

\subsection{Physiologie des Wärmehaushaltes}

\subsubsection{Grundlagen}

Beim Menschen wird die Körperkerntemperatur unter normalen Bedingungen auch dann in einem konstanten Bereich zwischen 36,5 und $37,5{ }^{\circ} \mathrm{C}$ gehalten, wenn die umgebenden Temperaturen deutlich niedriger oder höher sind (Guyton und Hall 1996). Der Aufrechterhaltung dieses engen Temperaturbereiches durch das autonome Nervensystem kommt bei Säugetieren, wie dem Menschen, eine entscheidende Bedeutung zu, da bereits kleine Abweichungen von der normalen Körperkerntemperatur zu Dysfunktionen von Körperzellen und Geweben führen (Hervey 1988, Sessler 1994).

Die Regulation des Wärmehaushaltes erfolgt dabei durch drei Komponenten im autonomen Nervensystem: 1. Die afferenten thermischen Signale, 2. eine Regulierung auf zentraler Ebene und 3. die Antwort über efferente Nervenfasern. Durch das Zusammenspiel dieser Teile des autonomen Nervensystems wird die Aufrechterhaltung der normalen Körperkerntemperatur sichergestellt (Daniel und Sessler 1997).

\subsubsection{Die afferenten Signalwege}

Die afferenten Signale werden durch peripher und zentral verteilte Wärme- und KälteRezeptoren an den Hypothalamus als zentrale Schaltstelle der Thermoregulation weitergeleitet. Sowohl die Wärme-, als auch die Kälterezeptoren sind dabei im ganzen Körper verteilt. Viele dieser Rezeptoren übertragen jedoch nicht nur thermale Reize, sondern zudem auch mechanische Reize (Pierau und Wurster 1981, Guyton und Hall 1996). In der Haut des Menschen sind sowohl Kälte-, als auch Wärmerezeptoren vorhanden. Die Anzahl der Kälterezeptoren ist jedoch um den Faktor 10 gegenüber der Anzahl der Wärmerezeptoren höher (Poulos 1981, Hervey 1988). 
Die Wärmerezeptoren haben ihren sensibelsten Bereich, in dem die Entladung am höchsten ist, in einem Bereich von $45-50{ }^{\circ} \mathrm{C}$ (Pierau und Wurster 1981), während die Kälterezeptoren die maximale Entladungsrate zwischen $25-30{ }^{\circ} \mathrm{C}$ aufweisen (Hervey 1988).

Den Kälterezeptoren in der Haut kommt bei der Sicherstellung der gleichbleibenden Körperkerntemperatur eine entscheidende Bedeutung zu. So konnte Benzinger in Versuchen an menschlichen Probanden nachweisen, dass die Kälterezeptoren in der Haut und ihre afferenten Signale an den Hypothalamus den entscheidenden Schritt zum Start von thermoregulativen Vorgängen bei Kälteexposition darstellen (Benzinger 1969).

\subsubsection{Die zentrale Regulierung}

In der anterioren Region des Hypothalamus werden die afferenten Impulse der Kälte- und Wärmerezeptoren verarbeitet. Der posteriore Hypothalamus regelt dagegen die gegenregulatorischen Veränderungen, die über absteigende Signalwege vermittelt werden (Buggy und Crossley 2000). In der präoptischen Region des Hypothalamus befinden sich Temperatur-sensitive und Temperatur-nicht-sensitive Neurone. Die Temperatur-sensitiven Neurone können wiederum in kalt- und warm-sensible Neurone unterschieden werden. Darüber hinaus befinden sich thermosensible Neurone auf Rückenmarksebene, in der Formatio reticularis und im posterioren Hypothalamus (Dickenson 1977).

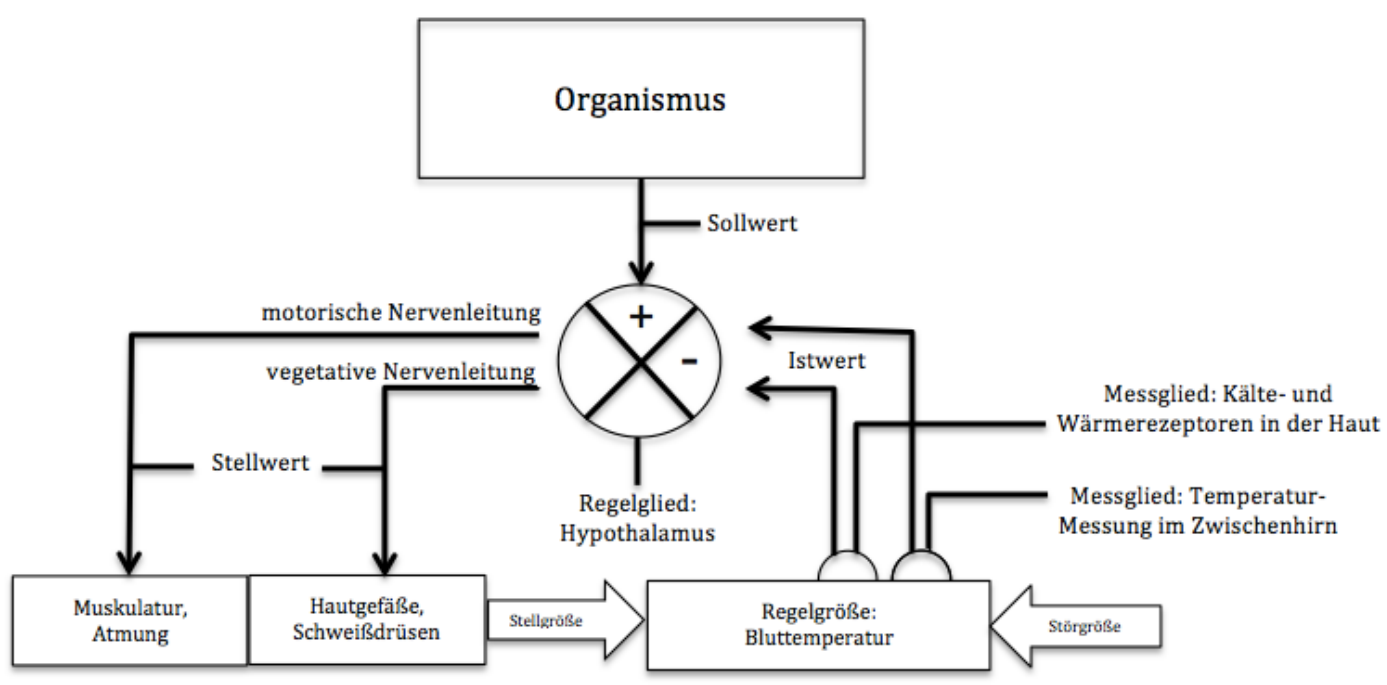

Abbildung 1: Regelkreis der Thermoregulation (A. Lorey-Tews) 


\subsubsection{Die efferenten Signalwege}

Die thermoregulatorische Antwort des Körpers erfolgt über 3 Mechanismen:

1. Durch die Änderung des Verhaltens.

Diese stellt die wirkungsvollste Antwort auf einen Kälte- oder Wärmereiz dar. Durch Anlegen warmer Kleidung, die Aufnahme von willkürlicher Bewegung oder die Anpassung der Raumtemperatur, gelingt es dem Menschen, auch unter extremen Umgebungsbedingungen, zu existieren (Wyss et al. 1975).

2. Durch die Vasomotion von arterio-venösen Shunts in der Haut.

Die Vasokonstriktion wird über $\alpha_{1}$ - und $\alpha_{2}$-adrenerge Rezeptoren vermittelt. Zusätzlich zu der beim Menschen jedoch vernachlässigbaren Aufrichtung der Haarzellen als Antwort auf einen Kältereiz (Flavahan 1991). Als Antwort auf einen Wärmereiz erfolgen die Vasodilatation und das Schwitzen (Wyss et al. 1975).

3. Durch das Shivering.

Shivering ist eine unwillkürliche Muskelbewegung. Dabei wird die metabolische Rate um den Faktor zwei bis drei gegenüber dem normalen Wert gesteigert. Hierüber kommt es zu einer aktiven Produktion von Wärme (Just et al. 1992).

Eine Wärmeproduktion ohne Shivering erfolgt lediglich bei Kindern durch das braune Fettgewebe. Bei Erwachsenen ist dieses jedoch zu vernachlässigen (Dawkins und Scopes 1965, Brück et al. 1976).

\subsection{Physikalische Grundlagen des Wärmehaushaltes}

\subsubsection{Temperatur und Temperaturempfinden}

Temperatur: von temperare (lat.) „ins richtige Mischverhältnis bringen“.

Die Temperatur ist eine definierte physikalische Zustandsgröße. Sie ist der messbare thermische Zustand eines Stoffes. Im medizinischen Bereich wird sie in Grad Celcius $\left(C^{\circ}\right)$ angegeben und erfolgt mit Hilfe von Thermometern. So kennzeichnet die Temperatur die Möglichkeit, innere Energie in Form von Wärme abzugeben. Das Temperaturempfinden des Menschen beruht nicht nur auf der Temperatur, sondern hängt auch von der körperlichen Aktivität und dem Wärmefluss ab (Tritthart 2011). 


\subsubsection{Wärme}

Wärme ist thermische Energie, die über eine Systemgrenze hinweg transportiert wird (Tritthart 2011).

\subsubsection{Wärmefluss}

Der Wärmefluss $(\dot{Q})$ ist eine physikalische Größe zur quantitativen Beschreibung von Wärmeübertragungsvorgängen. $\mathrm{Er}$ ist definiert als die in der Zeit ( $\delta t)$ übertragene Wärmemenge $\delta Q$ :

$$
I=\dot{Q}=\frac{\delta Q}{\delta t}
$$

Werden zwei Körper, die unterschiedliche Temperaturen aufweisen, in Kontakt gebracht, fließt solange Wärme vom Körper höherer Temperatur zum Körper, der über eine niedrigere Temperatur verfügt, bis beide Körper die gleiche Temperatur besitzen. Die Endtemperatur liegt dabei nie außerhalb der beiden Ausgangstemperaturen der Körper (Gunga 2013).

\subsubsection{Wärmekapazität}

Die Wärmekapazität eines Körpers gibt das Verhältnis von Wärmeänderung und Temperaturänderung an. Sie gibt an, wieviel Energie notwendig ist, um die Temperatur eines Objekts anzuheben bzw. wieviel Energie abgegeben wird, wenn die Temperatur eines Körpers fällt. Dabei hängt die Endtemperatur von den spezifischen Wärmekapazitäten der beteiligten Systeme ab.

Die spezifische Wärmekapazität gibt an, wieviel Energie einem Kilogramm eines Stoffes zugeführt werden muss, um seine Temperatur um ein Grad Celcius $\left(C^{\circ}\right)$ zu erhöhen (Tritthart 2011, Seibt 2009).

Beispielhaft sind folgende spezifische Wärmekapazitäten genannt:

Menschlicher Körper (Burton 1935):

$3,475 \mathrm{~kJ}^{\circ} \mathrm{C}^{-1} \mathrm{~kg}^{-1}$

Kristalloide Lösung (von Hintzenstern et al. 2004):

$4,18 \mathrm{~kJ}^{\circ} \mathrm{C}^{-1} \mathrm{~kg}^{-1}$ 


\subsubsection{Physikalische Mechanismen des Wärmeaustausches}

Der menschliche Körper kann über 4 Mechanismen mit seiner Umgebung Wärme austauschen:

- Radiation

- Konduktion

- Konvektion

- Evaporation

\subsubsection{Radiation}

Radiation bezeichnet die Wärmestrahlung. Wärme wird durch elektromagnetische Wellen (infrarote Strahlung, infrarotes Licht) übertragen. Die Wärmestrahlung wird hierbei vom menschlichen Körper absorbiert oder abgegeben. Diese Form der Wärmeübertragung benötigt kein Medium zur Wärmeübertragung und funktioniert somit im Unterschied zur Wärmeleitung und Wärmeströmung auch im Vakuum (Gunga 2013).

\subsubsection{Konduktion}

Die Konduktion wird auch Wärmediffusion oder Wärmeleitung genannt. Die Konduktion bezeichnet den Wärmefluss in oder zwischen zwei Feststoffen infolge eines Temperaturunterschiedes. Die Wärme fließt hierbei immer vom Ort der höheren Temperatur zum Ort der geringeren Temperatur. Die Wärme wird dabei von Molekül zu Molekül übertragen. Die Moleküle verändern jedoch nicht ihren Standort (Gunga 2013).

Ein Beispiel für die innerklinische Anwendung sind elektrisch oder wasserbetriebene Wärmematten zur Wärmung von Patienten.

\subsubsection{Konvektion}

Konvektion: von convectum (lat.) „mitgetragen“.

Der Begriff der thermischen Konvektion bezeichnet das Mitführen von thermischer Energie. Im klinischen Alltag bedienen sich konvektive Luftwärmer zur Wärmung von Patienten dieses Prinzips. Durch eine Ortsveränderung von leicht beweglichen Teilchen, die gespeicherte 
Wärme mit sich führen, kommt es zu einer Steigerung der Energie und Temperatur von umströmten Körpern. Konvektion findet daher nur in Gasen oder Flüssigkeiten statt.

Die im Körperinneren ständig produzierte Wärme gelangt sowohl konduktiv, als auch konvektiv mit dem Blutstrom zur Körperoberfläche (Persson 2011).

\subsubsection{Evaporation}

Als Evaporation wird das Prinzip der Verdunstung bezeichnet. Medizinisch relevante Beispiele sind z.B. Wärmeverluste durch eröffnete Körperhöhlen während Operationen oder als Folge von Verletzungen. Aufgrund der hohen Verdampfungswärme des Wassers, bildet die Verdunstung von Wasser an den Oberflächen der Haut und der Schleimhäute einen wesentlichen Vorgang im Rahmen der Wärmeabgabe. Dieses geschieht durch den Übergang vom flüssigen in den gasförmigen Zustand (vom hohen Wasserdampfpartialdruck zum niedrigen Wasserdampfpartialdruck). Durch die Erhöhung der Luftgeschwindigkeit kommt es zudem zu einer Erhöhung der Wärmeabgabe durch Evaporation. Dabei besteht ein linearer Zusammenhang zwischen evaporativem und konvektivem Wärmeverlust (Persson 2011).

\subsection{Hypothermie}

In der Literatur wird bei Traumapatienten übereinstimmend ein Abfall der Körperkerntemperatur unter $35{ }^{\circ} \mathrm{C}$ als Beginn der Hypothermie gesehen (Danzl und Pozos 1994, Shafi et al. 2005, Beilman et al. 2009, Mommsen et al. 2012, Trentzsch et al. 2012). Die Ursachen der Hypothermie sind vielfältig. Grundsätzlich kann die Hypothermie in drei Hauptursachen unterteilt werden (Seekamp et al. 1999, Mccullough und Arora 2004).

Tabelle 1 Ursachen der Hypothermie

\begin{tabular}{||l|l|c||}
\hline \hline ENDOGENE HYPOTHERMIE & $\begin{array}{l}\text { KONTROLLIERT INDUZIERTE } \\
\text { HYPOTHERMIE }\end{array}$ & AKZIDENTELLE HYPOTHERMIE \\
\hline dermale & innerklinische Hypothermie- & iatrogene Ursachen \\
Schäden/Erkrankungen & behandlungen & aggressives \\
$\bullet \quad$ Verbrennungen & $\bullet$ post Reanmima- & Flüssigkeits- \\
$\bullet \quad$ exfoliative & tionem & management \\
\hline
\end{tabular}




\begin{tabular}{|c|c|c|}
\hline 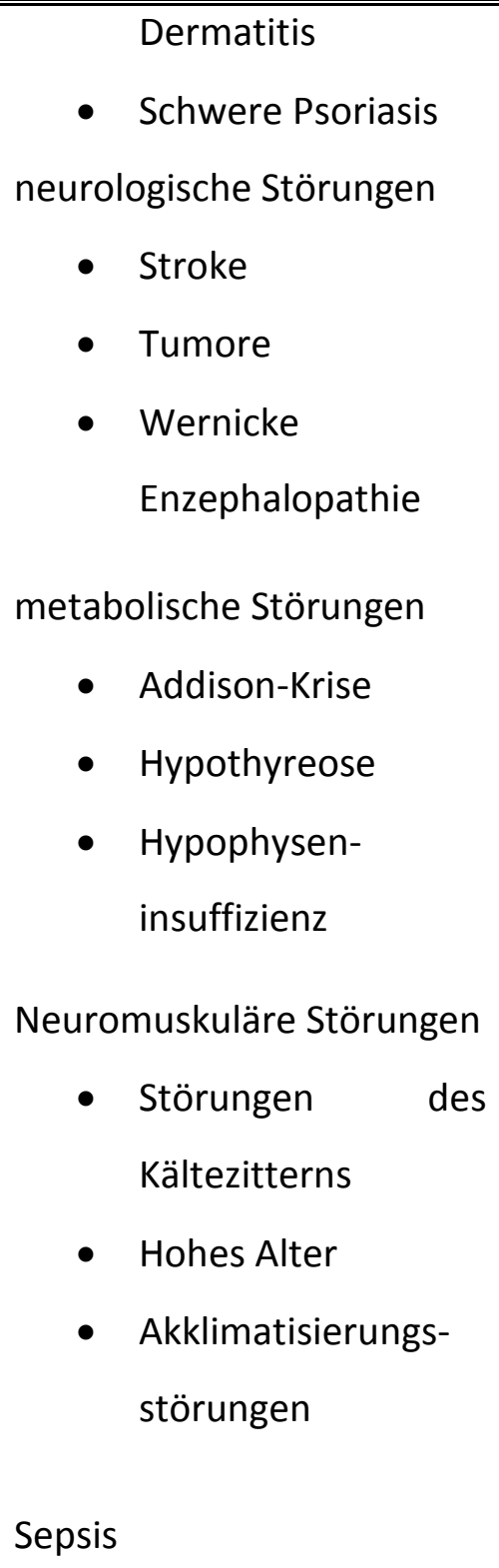 & $\begin{array}{l}\text { - intraoperative } \\
\text { Hypothermie- } \\
\text { behandlung } \\
\text { (bspw. } \\
\text { Kardiochirurgie) }\end{array}$ & $\begin{array}{l}\text { Medikamenten-, Drogen- } \\
\text { induziert } \\
\text { - } \quad \text { Alkohol } \\
\text { - Sedativa/ Hypnotika } \\
\text { - Neuroleptika } \\
\text { (Phenothiazine) } \\
\text { Umwelteinflüsse } \\
\text { - Kälteexposition } \\
\text { - Wasserunfälle } \\
\text { Trauma } \\
\text { - Polytrauma } \\
\text { - Schädel-Hirn-Trauma } \\
\text { - Wirbelsäulentrauma }\end{array}$ \\
\hline
\end{tabular}

\subsection{Hypothermie und Trauma}

Jährlich werden über 67.000 Personen als Folge von Verkehrsunfällen schwer verletzt. Rund 16.000 erleiden dabei so schwere Verletzungen, dass sie einen Injury Severity Score $\geq 16$ aufweisen und somit die Kriterien des Polytraumas erfüllen (Copes et al. 1988, Jahresbericht Traumaregister 2015).

Zudem starben 2015 in Deutschland als Folge eines Verkehrsunfalles 3.459 Personen (Statistisches Bundesamt 2015). Durch das statistische Bundesamt werden die als Folge von Verkehrsunfällen anfallenden Behandlungskosten mit jährlich 13,5 Milliarden Euro angegeben. Der Gesamtschaden, in dem auch Tod und Invalidität Berücksichtigung finden, beträgt 41 Milliarden Euro jährlich (Statistisches Bundesamt 2015). 
60\% der Todesopfer im Straßenverkehr versterben bereits während der präklinischen Phase (Christ und Lackner 2004). Der hämorrhagische Schock als Folge eines Traumas ist dabei die häufigste Ursache vermeidbarer Todesfälle (Hess et al. 2008).

Eine frühe und zielgerichtete Bekämpfung von Blutungen und Störungen der Hämostase kann helfen, die traumaassoziierte Sterblichkeit zu senken (Riskin et al. 2009).

Die präklinische Zeit, also die Dauer zwischen Unfallereignis und Klinikaufnahme, ist in den vergangenen 10 Jahren nahezu unverändert geblieben und betrug 2015 laut Daten des Traumaregisters der Deutschen Gesellschaft für Unfallchirurgie (DGU) im Mittel $71 \pm 56$ min ( $n=12106)$ (Jahresbericht Traumaregister 2015). Den in diesem Zeitraum durchgeführten Maßnahmen zur Bekämpfung des Schockgeschehens kommt somit eine besondere Bedeutung zu.

\subsubsection{Prävalenz}

Die akzidentelle Hypothermie als Folge eines schweren Traumas wird dabei häufig beobachtet (Weuster et al. 2016): Die deutsche Arbeitsgruppe Mommsen et al. konnte nach der Auswertung von 310 polytraumatisierten Patienten nachweisen, dass mehr als $36 \%$ der Patienten während des Zeitraums der Einlieferung in den Schockraum und dem Beginn der primären operativen Versorgung eine Körperkerntemperatur unter $35{ }^{\circ} \mathrm{C}$ aufwiesen (Mommsen et al. 2013). Andere internationale Autoren zeigten bei bis $\mathrm{zu} 10 \%$ aller Traumapatienten, unabhängig von der Verletzungsschwere, einen hypothermen Zustand (Martin et al. 2005, Wang et al. 2005, Aitken et al. 2009, Wade et al. 2011, Trentzsch et al. 2012)

Jurkovich et al. und auch Seekamp et al. konnten zudem nachweisen, dass eine schwere Hypothermie mit einer signifikant höheren Inzidenz von Verletzungen assoziiert ist (Jurkovich et al. 1987, Seekamp et al. 1996).

Nach Auswertung aller Studien ist jedoch festzustellen, dass der Körperkerntemperatur in der Frühphase der Traumaversorgung eine unzureichende Beachtung zukommt. Betrachtet man die eingeschlossenen Daten, so fällt auf, dass große Kollektive der Patienten leider nicht eingeschlossen werden konnten, da keine Temperaturkontrolle in der präklinischen und frühen innerklinischen Phase der Traumaversorgung durchgeführt wurde (Aitken et al. 2009, Wade et al. 2011, Trentzsch et al. 2012). 


\subsubsection{Ursachen des Wärmeverlustes nach schwerem Trauma}

Dass eine schwere traumatische Verletzung häufig mit einer Hypothermie einhergeht, ist nicht nur einem Umstand geschuldet, sondern die Folge vieler Einflüsse und Faktoren (Hildebrand et al. 2009).

Die Hypovolämie und der daraus resultierende Schock, werden bei polytraumatisierten Patienten häufig beobachtet. Der hypovolämische Schock geht typischerweise mit dem Verlust der Thermoregulation einher (Tsuei und Kearney 2004). Seekamp et. al. konnten zeigen, dass es durch die Sympatikus-Aktivierung, die letztlich einen erhöhten Verbrauch von Sauerstoff und Energiereserven zur Folge hat, zu einem signifikant erniedrigten ATP-Spiegel im Plasma kommt. Grund hierfür ist die durch das Schockgeschehen bereits bestehende Sauerstoffschuld in der Peripherie. Hierdurch kommt es letztlich zum Versagen dieser gegenregulatorischen Bemühungen und ein Temperaturverlust ist die Folge (Seekamp et al. 1999). Zur Bekämpfung der Kreislaufinsuffizienz verabreichte kalte Infusionen tragen ebenfalls zum Absinken der Körpertemperatur bei (Fritz et al. 1998). Die durchschnittliche präklinisch verabreichte Infusionsmenge wird nach Daten des Deutschen Traumaregisters mit $698 \mathrm{ml}$ angegeben (Jahresbericht Traumaregister 2015).

Weitere iatrogene Ursachen während der Traumaversorgung aggravieren die präklinische Hypothermie. So konnten Langhelle et al. zeigen, dass Patienten, die präklinisch analgosediert wurden ( $n=207)$, bei Ankunft im Schockraum signifikant häufiger eine Körpertemperatur unter $35{ }^{\circ} \mathrm{C}$ aufwiesen, als die Patienten, die nicht anästhesiert wurden ( $n=287$ ) (Langhelle et al. 2012). Diese Effekte können auch innerklinisch beobachtet werden. So kommt es nach Induktion einer Narkose durch die Weitstellung der peripheren Gefäße zu einer Umverteilung der Körperwärme und zu einem Absinken der Körpertemperatur (Hildebrand et al. 2004). Nach Daten des Deutschen Traumaregisters wurden 2014 23,0\% aller im Traumaregister erfassten schwerverletzten Patienten bereits am Unfallort intubiert (Jahresbericht Traumaregister 2015). Auch das Entkleiden und präklinisch durchgeführte invasive Maßnahmen, wie die Anlage einer Thoraxdrainage, können zu einem Abfall der Körpertemperatur führen. Die präklinische Anlage einer Thoraxdrainage erfolgte bei 2,8\% aller Patienten, die im Deutschen Traumaregister erfasst wurden (Jahresbericht Traumaregister 2015). 
Die Jahreszeit und die klimatischen Gegebenheiten haben jedoch kaum Einfluss auf die Entstehung der Hypothermie beim schweren Trauma. So wurden in der Literaturrecherche überwiegend Arbeiten gefunden, die keinen Zusammenhang zwischen den Jahreszeiten zeigten (Rutherford et al. 1998, Sundberg et al. 2011, Langhelle et al. 2012). Aitken et al. konnten auch in einer subtropischen Umgebung nachweisen, dass es abhängig von der Schwere der Verletzung zu einer Hypothermie kam (Aitken et al. 2009). Lediglich in einer Arbeit von Ireland et al. zeigte sich eine signifikante Häufigkeit in den Wintermonaten (Ireland et al. 2011).

\subsubsection{Einflüsse der traumainduzierten Hypothermie}

Die Einflüsse der Hypothermie beim Schwerverletzten sind vielfältig. Insbesondere für eine funktionierende Hämostase bedarf es physiologischer Bedingungen im menschlichen Körper. Die wichtigsten Bausteine einer funktionierenden Gerinnung sind ein ausgeglichener SäureBasen-Haushalt, ausreichend hohes Calcium, der Hämatokrit und eine physiologische Körpertemperatur (Lier et al. 2008). Kommt es zu einem gleichzeitigen Auftreten von Koagulopathie, Azidose und Hypothermie, ist die Letalität des Patienten deutlich gesteigert. Der Begriff lethal triad wird hierfür in der Literatur benutzt (Tsuei und Kearney 2004, Kheirbek et al. 2009, Mitra et al. 2012). Die Hypothermie beeinflusst dabei sowohl den plasmatischen als auch den zellulären Anteil der Gerinnung (Hildebrand et al. 2004) (Park et al. 2013). Die an der Gerinnung beteiligten Proteasen funktionieren nur in einem definierten Temperaturbereich vollständig. Kommt es zu einem Abfall der Körpertemperatur, so nimmt auch die Aktivität dieser Enzyme ab. So kommt es bei einer Temperaturabnahme von $1^{\circ} \mathrm{C} z u$ einer Abnahme der Aktivität der Gerinnungsproteasen um 10\% (Lier et al. 2007). Unterhalb von $33{ }^{\circ} \mathrm{C}$ ist die Aktivität der Gerinnungsfaktoren derart erniedrigt, dass sie funktionell einem Faktorenmangel von 50\% entspricht (Johnston et al. 1994). Am stärksten ist jedoch der Einfluss der Temperatur auf die Thrombozyten. Bereits unterhalb von $35^{\circ} \mathrm{C}$ kommt es zu einem verstärkten Pooling der Thrombozyten in der Milz und in der Leber, eine Verminderung der im Plasma vorhandenen Thrombozyten ist die Folge (Kermode et al. 1999). Unterhalb von $34^{\circ} \mathrm{C}$ kommt es zu einer Blockierung der Wechselwirkung zwischen dem GP-Ib-IX-V-Komplex und dem von-Willebrand-Faktor. Hierdurch ist eine Verminderung der Adhäsion und Aggregation der Thrombozyten die Folge (Kermode et al. 1999). 
Zudem wird durch die Hypothermie die Fibrinogensynthese gehemmt (Martini 2009). Gleichzeitig kommt es zu einer erhöhten fibrinolytischen Aktivität. Grund hierfür ist eine verminderte Ausschüttung von plasminogen-activator-inhibitor und Alpha-2-Antiplasmin (Yenari et al. 1995).

Aber auch auf das Immunsystem hat die Hypothermie bei schwerverletzten Patienten einen entscheidenden Einfluss. So konnte gezeigt werden, dass Patienten, die während der primären Phase der Traumaversorgung eine Hypothermie erleiden, signifikant häufiger im Verlauf Wundinfektion, Pneumonien bis hin zur Sepsis aufweisen (Manson et al. 2012, Seamon et al. 2012, Trentzsch et al. 2012). Hierfür ist der Einfluss der Hypothermie auf die proinflammatorischen als auch auf die antiimflammatorischen Zytokine verantwortlich (Hildebrand et al. 2005).

Ob die akzidentelle Hypothermie nach schwerem Trauma einen unabhängigen Faktor für eine gesteigerte Mortalität darstellt oder nur als Surrogatparameter der Schwere der Verletzung und Hämorrhagie begriffen werden muss, wird in der Literatur uneinheitlich dargestellt.

So konnten zum einen viele Autoren in ihren Studien die Hypothermie als unabhängigen Mortalitätsfaktor ausmachen. In ihren Studien zeigten Patienten mit tödlichen Verläufen eine signifikant niedrigere Körpertemperatur, höhere Verletzungsgrade und einen gesteigerten Bedarf an Transfusion von Blutprodukten (Jurkovich et al. 1987, Bernabei et al. 1992, Gentilello et al. 1997, Martin et al. 2005, Shafi et al. 2005, Wang et al. 2005, Aitken et al. 2009, Inaba et al. 2009).

Einige Autoren beschreiben zudem, dass bereits eine Körpertemperatur von $<35{ }^{\circ} \mathrm{C}$, zum Zeitpunkt der Einlieferung im Krankenhaus, einen unabhängigen Mortalitätsfaktor darstellt (Martin et al. 2005, Wang et al. 2005, Aitken et al. 2009, Ireland et al. 2011).

Andere Autoren konnten dagegen keinen Zusammenhang zwischen der Hypothermie und gesteigerter Mortalität ausmachen (Steinemann et al. 1990, Beilman et al. 2009, Mommsen et al. 2013). So konnten Trentzsch et al. in einer Auswertung von über 5.000 Patienten keine unabhängige Verbindung zwischen Mortalität und Hypothermie feststellen. Als Ergebnis ihrer Arbeit sahen die Autoren die Hypothermie als Folge der Schwere der Verletzung und der Hämorrhagie und empfehlen die regelhafte und standardisierte Messung der Körpertemperatur, um hierdurch Hinweise auf die Schwere der Verletzung zu gewinnen. 


\subsection{Wärmeprotektive Verfahren}

Zur Vermeidung von perioperativen hypothermen Zuständen stehen zahlreiche wärmeprotektive Verfahren zur Verfügung, welche in aktive und passive Wärmemethoden unterschieden werden können. Ihre Effektivität ist Gegenstand vieler Studien gewesen. Bräuer et al. haben die Ergebnisse in folgender Tabelle zusammengeführt (Bräuer et al. 2006):

Tabelle 2: Vergleich der perioperativen wärmeprotektiven Maßnahmen (Bräuer et al. 2006)

\begin{tabular}{|c|c|c|c|c|}
\hline $\begin{array}{l}\text { WÄRMEPROTEKTIONS- } \\
\text { VERFAHREN }\end{array}$ & EFFEKTIVITÄT & $\begin{array}{l}\text { AUFWAND BZW. } \\
\text { PRAKTIKABILITÄT }\end{array}$ & KOSTEN & LIMITIERUNGEN \\
\hline $\begin{array}{l}\text { Erhöhung der } \\
\text { Raumtemperatur }\end{array}$ & Gut & Gering & Niedrig & $\begin{array}{l}\text { Toleranz der } \\
\text { Operateure }\end{array}$ \\
\hline Atemgasklimatisierung & Gering & Gering & Niedrig & Wenig effektiv \\
\hline Ösophaguswärmer & Gering & Mäßig & Mäßig & Wenig effektiv \\
\hline $\begin{array}{l}\text { Heizmatten unter dem } \\
\text { Rücken }\end{array}$ & Gering & Gering & Mäßig & Wenig effektiv \\
\hline $\begin{array}{l}\text { Heizmatten auf dem } \\
\text { Körper }\end{array}$ & Sehr gut & $\begin{array}{l}\text { Mäßig bis hoch je } \\
\text { nach System }\end{array}$ & $\begin{array}{l}\text { Mäßig bis hoch je } \\
\text { nach System }\end{array}$ & Teilweise sehr teuer \\
\hline konvektive Luftwärmer & Sehr gut & $\begin{array}{l}\text { Mäßig; Viele } \\
\text { verschiedene Decken } \\
\text { vorhanden }\end{array}$ & Mäßig & $\begin{array}{l}\text { Ohne Vorwärmung } \\
\text { nicht immer } \\
\text { ausreichend }\end{array}$ \\
\hline Isolation & Gut & Gering & Mäßig & $\begin{array}{l}\text { Zurzeit keine guten } \\
\text { Materialien für den } \\
\text { OP vorhanden }\end{array}$ \\
\hline Infrarotstrahler & Gut & Gering & Mäßig & $\begin{array}{l}\text { Wärmt auch die } \\
\text { Mitarbeiter im OP } \\
\text { Nutzbare Fläche } \\
\text { klein }\end{array}$ \\
\hline Infusionswärmer & Gut & Mäßig & $\begin{array}{l}\text { Mäßig bis hoch je } \\
\text { nach System }\end{array}$ & $\begin{array}{l}\text { Nur bei hohem } \\
\text { Umsatz effektiv }\end{array}$ \\
\hline $\begin{array}{l}\text { Präoperative Gabe von } \\
\text { Aminosäuren }\end{array}$ & Gut & Mäßig & Mäßig & $\begin{array}{l}\text { Benötigt sicheres } \\
\text { OP-Programm }\end{array}$ \\
\hline Vorwärmung & Sehr gut & $\begin{array}{l}\text { Schwierig zu } \\
\text { organisieren }\end{array}$ & $\begin{array}{l}\text { Gering, da das } \\
\text { Material } \\
\text { weiterverwendet } \\
\text { wird }\end{array}$ & $\begin{array}{l}\text { Benötigt sicheres } \\
\text { OP-Programm, } \\
\text { Erfordert viel } \\
\text { Mitarbeit. }\end{array}$ \\
\hline
\end{tabular}

Viele der aufgeführten Wärmeverfahren sind für den präklinischen Einsatzbereich nicht durchführbar oder sind aufgrund der bereits innerklinisch fehlenden Effektivität nicht geeignet.

Den innerklinischen Standard im Wärmemanagement bildet der konvektive Luftwärmer (Hynson et al. 1993, Bennett et al. 1994, Bock et al. 1998, Bräuer et al. 2000). Seine Effizienz ist in vielen Studien gut belegt (Scherer 1997, Heuer 2000, Bräuer et al. 2002). 


\subsection{Wärmeprotektive Verfahren in der Präklinik}

Bisher finden sich nur wenige Studien über die Wirksamkeit von wärmeprotektiven Verfahren unter präklinischen Bedingungen. Hiervon behandelt der überwiegende Anteil der Studien den Einsatz von passiven Wärmemethoden.

So überprüften Thomassen et al. in einer prospektiven Studie an acht freiwilligen, gesunden Probanden den Effekt von drei verschiedenen Isolationsmethoden und konnten hierbei zeigen, dass eine Kombination zweier verschiedener Isolationsmethoden (=Hibler's Method), die mittlere Hauttemperatur signifikant ansteigen ließ (Thomassen et al. 2011).

Henriksson et al. konnten nachweisen, dass die von Rettungsdiensten genutzten PolyesterDecken unter windigen Bedingungen keinen wirksamen Schutz gegen Temperaturverluste bieten (Henriksson et al. 2009).

Sterba et al. untersuchten unter kontrollierten Bedingungen an acht Freiwilligen, ob zwei unterschiedliche „Rescue-sleeping-bags“ eine effiziente Steigerung der Körperkerntemperatur nach vorausgegangener akzidenteller Hypothermie durchführen können. Obwohl bei dem einen Modell sogar aktiv Wärme durch einen batteriebetriebenen Föhn über Luftschläuche an der Brust, der Axilla und am Nacken zugeführt wurde, konnte keine signifikante Steigerung der Körperkerntemperatur beobachtet werden (Sterba 1991).

Eine weitere Studie untersuchte 30 Probanden in einer Umgebungstemperatur von $4{ }^{\circ} \mathrm{C}$ und verglich die Wärmung mittels eines Halogenstrahlers, wie er von Feuerwehren zur Ausleuchtung von Unfallstellen mitgeführt wird, mit der Wirkung eines konvektiven Luftwärmers, wie er zum Beheizen von Zelten o.ä. Verwendung findet. Hierbei wurde die gewärmte Luft unter eine Plastikfolie gelenkt. Es zeigten sich hochsignifikante Vorteile des konvektiven Luftwärmers, bei vollständig fehlendem Effekt des Halogenstrahlers. Jedoch konnte aufgrund der hohen Temperatur an der Öffnung der ausströmenden Luft keine generelle Empfehlung für diese Maßnahme ausgesprochen werden (Jebens 2014).

Auch in einer stichprobenartig durchgeführten Recherche in den gängigen Lehrbüchern zur präklinischen Traumaversorgung findet sich stets der Hinweis, einen Wärmeverlust mittels Decken o.ä. zu verhindern (Luxem et al. 2010, NAEMT 2009) Auf Möglichkeiten eines aktiven Wärmemanagements wird jedoch nur in einem Buch verwiesen (Südmersen und Heyne 2008). 


\subsection{Fragestellung}

Die akzidentelle Hypothermie nach schwerem Trauma wird häufig beobachtet und bringt zahlreiche Komplikationen im weiteren klinischen Behandlungsverlauf mit sich. Basierend auf dem Wissen, dass eine frühzeitige aktive Wärmetherapie zur Verhinderung eines hypothermen Zustandes einfacher und effizienter ist als die Wiedererwärmung eines bereits hypothermen Patienten, ergibt sich die Notwendigkeit einer sicheren und effizienten Wärmemethode, die bereits präklinisch zur Anwendung kommt.

Für den innerklinischen Bereich stehen entsprechende konvektive Luftwärmer zur Verfügung. Untersuchungen zur Anwendung dieser Geräte in einem präklinischen Setting fehlen jedoch bisher. Mit Hilfe dieser Studie sollen daher zu folgenden Fragestellungen Erkenntnisse gewonnen werden:

1. Ist bei kälteexponierten Personen durch den Einsatz von konvektiven Luftwärmern, welche ursprünglich für den innerklinischen Einsatz vorgesehen sind, eine Steigerung bzw. Stabilisierung der Körperkerntemperatur, der mittleren Hauttemperatur oder der Gesamtkörperwärmemenge möglich?

2. Ist unter diesen Bedingungen durch den Einsatz von Vlies-Einmaldecken, die für den präklinischen Einsatz vorgesehen sind, eine Steigerung bzw. Stabilisierung der Körperkerntemperatur, der mittleren Hauttemperatur oder der Gesamtkörperwärmemenge möglich?

3. Gibt es signifikante Unterschiede zwischen beiden Methoden? 


\section{Material und Methoden}

\subsection{Genehmigung der Ethik-Kommission}

Auf Antrag bescheinigte die Ethik-Kommission der Medizinischen Fakultät der Georg-AugustUniversität Göttingen am 13. Januar 2014 mit der Antragsnummer 6/8/11, dass gegen die Durchführung der Versuchsreihen zur „prospektiven Untersuchung zur Wirksamkeit von wärmeprotektiven Maßnahmen unter präklinischen Bedingungen" weder ethische, moralische noch rechtliche Bedenken bestehen.

\subsection{Material}

\subsubsection{Probandenkollektiv}

Für die Teilnahme an der Untersuchungsreihe konnten 32 Probanden gewonnen werden. Alle stellten sich freiwillig dieser Studie und willigten schriftlich in die Teilnahme ein. Zu jedem Zeitpunkt war es ihnen möglich, ohne eine Angabe von Gründen, die Versuche abzubrechen. Die Probanden wurden in einem persönlichen Gespräch über den Ablauf des Versuches und mögliche Risiken aufgeklärt. Zusätzlich wurde ihnen ein detaillierter Aufklärungsbogen ausgehändigt.

In der folgenden Tabelle sind die Kriterien dargestellt, die für eine Teilnahme zwingend erfüllt sein mussten (=Einschlusskriterien). Zudem werden die Kriterien aufgeführt, die zu einem Ausschluss der Probanden aus der Studie geführt hätten (=Ausschlusskriterien).

Tabelle 3 Einschluss- und Ausschlusskriterien Probandenkollektiv

\begin{tabular}{|c|c|}
\hline EINSCHLUSSKRITERIEN & AUSSCHLUSSKRITERIEN \\
\hline $\begin{array}{l}\text { - Volle Einwilligungsfähigkeit } \\
\text { - } \text { Verständnis der deutschen Sprache } \\
\text { - Alter }>18 \text { Jahre, }<65 \text { Jahre } \\
\text { - ASA-Klassifikation I-II } \\
\text { - ASA II = Normal gesunder Patient } \\
\text { gemeinerkrankung } \\
\text { - Body-Mass-Index }<30 \mathrm{~kg} / \mathrm{m}^{2}\end{array}$ & $\begin{array}{l}\text { - } \text { Alter }<18 \text { Jahre, }>65 \text { Jahre } \\
\text { - } \text { Herzrhythmusstörungen } \\
\text { - } \text { Hyper-/Hypothyreose } \\
\text { - } \text { Leberfunktionsstörungen } \\
\text { - } \quad \text { Nierenfunktionsstörungen } \\
\text { - } \text { Infekt der oberen Luftwege }\end{array}$ \\
\hline
\end{tabular}


14,5 und 9,5 Meter Höhe. Für die Versuchsreihe wurde in der Mitte des Kühlhauses eine Fläche von 10 x 15 Meter geschaffen. Hier wurde ein Fahrersitz eines PKW verbaut, um das Szenario eines Verkehrsunfalls mit Einklemmung authentischer nachempfinden zu können. Der Autositz war von allen Seiten aus zugänglich. Auf der linken Seite wurde der konvektive Luftwärmer platziert. Neben den im Folgenden beschriebenen Messgeräten, war auch zu jederzeit eine komplette Notfallausrüstung, bestehend aus Notfallkoffer, EKG und Defibrillator, vorhanden. Für die Vorbereitungen und Überwachung der Probanden, jeweils vor und nach dem Versuch standen beheizte Sozialräume zur Verfügung. Dort bestand auch die Möglichkeit, nach dem Versuch warme Getränke einzunehmen.

\subsubsection{Wärmegerät 1: Konvektiver Luftwärmer}

Als Wärmegerät 1 wurde das Patienten-Wärmesystem WarmTouch WT-5900 der Firma Nellcor gewählt. Es handelt sich hierbei um einen konvektiven Luftwärmer zur Prävention und Behandlung der Hypothermie bei Patienten im innerklinischen Bereich.

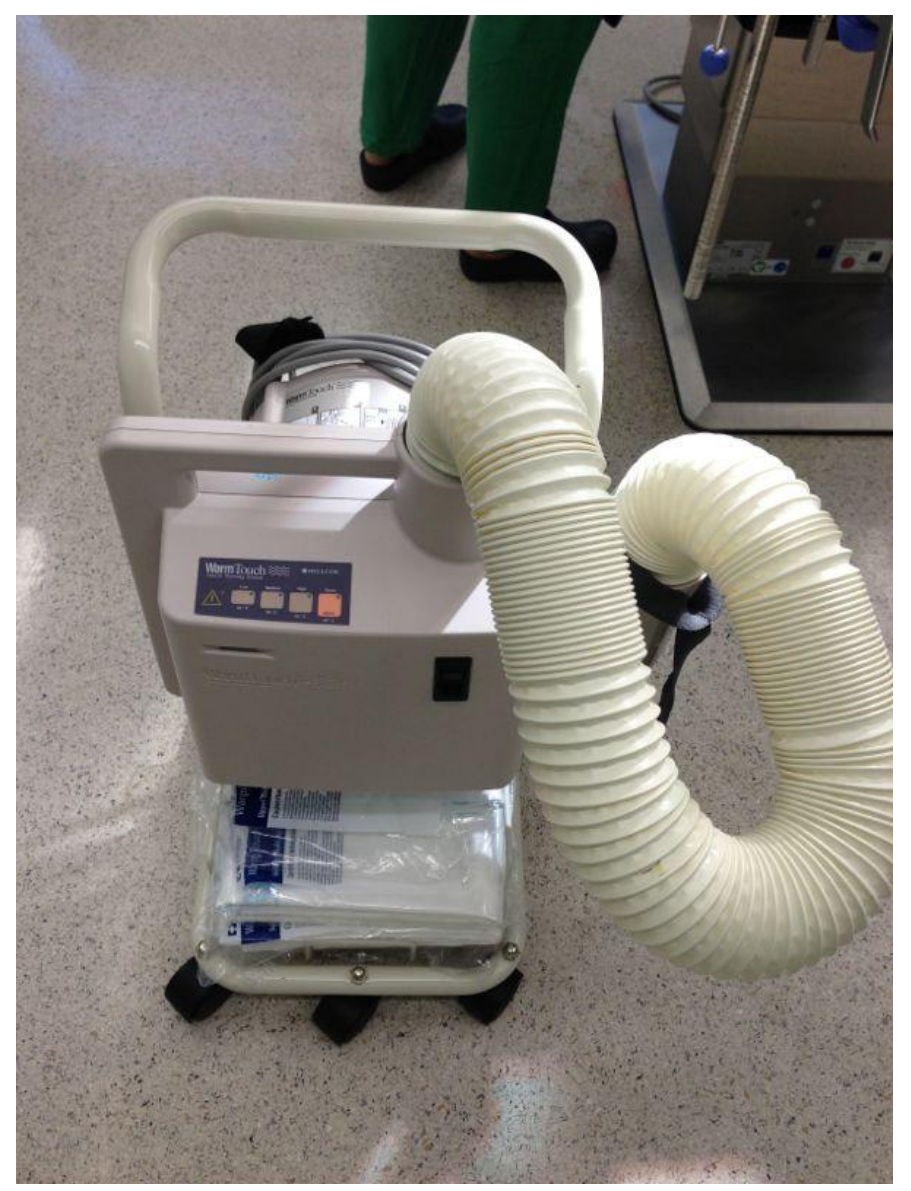

Abbildung 2: konvektiver Luftwärmer Warmtouch WT 5900 der Firma Nellcor. Quelle: A. Lorey-Tews 
Das Gerät erwärmt die Umgebungsluft und transportiert diese über einen Schlauch zum Patienten. Die Luft wird am Patienten über entsprechende Wärmedecken gleichmäßig auf der Oberfläche des Patienten verteilt. Als konvektive Wärmedecke wurde eine EinmalGanzkörper-Wärmedecke gewählt.

Am Gerät können folgende Temperaturbereiche ausgewählt werden. Die angegebene Temperatur gibt jeweils die durchschnittliche Temperatur der in den Schlauch einströmenden Luft an:

$\begin{array}{ll}\text { Niedrig: } & 32{ }^{\circ} \mathrm{C} \pm 1,5^{\circ} \mathrm{C} \\ \text { Mittel: } & 38{ }^{\circ} \mathrm{C} \pm 1,5^{\circ} \mathrm{C} \\ \text { Hoch: } & 43{ }^{\circ} \mathrm{C} \pm 1,5^{\circ} \mathrm{C} \\ \text { Boost: } & 45^{\circ} \mathrm{C} \pm 1,5^{\circ} \mathrm{C}\end{array}$

Bei Auswahl der Funktion „Boost“ arbeitet das Gebläse für 45 Minuten in dieser Leistungsstufe. Hiernach reduziert sich die Einstellung automatisch von „Boost“ zu „Hoch“. Während des Versuches lief das Gebläse jeweils durchgehend in der Einstellung „Boost“.

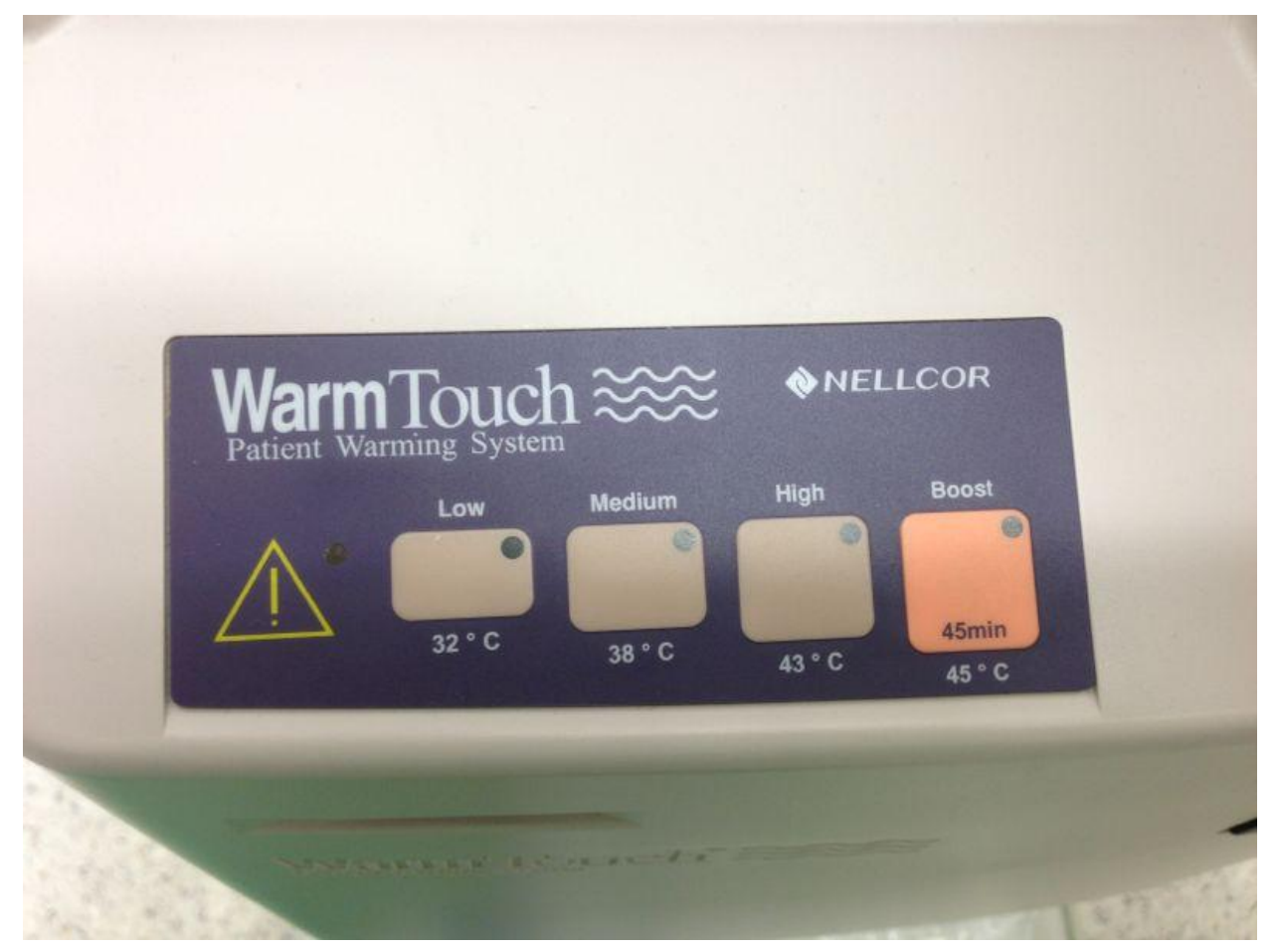

Abbildung 3: Bedienfeld des Warmtouch WT 5900 der Firma Nellcor. Quelle: A. Lorey-Tews

Das Gerät hat folgende Abmessungen: $38 \mathrm{~cm} \times 41 \mathrm{~cm} \times 21 \mathrm{~cm}(\mathrm{BxHxT})$ und wird mit 220V AC betrieben. 


\subsubsection{Wärmegerät 2: Vlies-Einmaldecke}

Als Wärmedecke wurde eine Einmaldecke der Firma Reintex gewählt. Die gewählte Decke wird laut Angaben des Herstellers für die Verwendung an Patienten bei Rettungsdiensten, Feuerwehren und Krankentransporten produziert (Reintex 2013). Es handelt sich hierbei um eine Einmaldecke mit einer Hülle aus einem PP (Polypropylen)-Vliesstoff und einer Füllung aus Polyesterwolle. Die Größe beträgt 1,90 m x 1,10 m. Das Gewicht der Vlies-Einmaldecke wird vom Hersteller mit ca. $250 \mathrm{~g}$ angegeben.

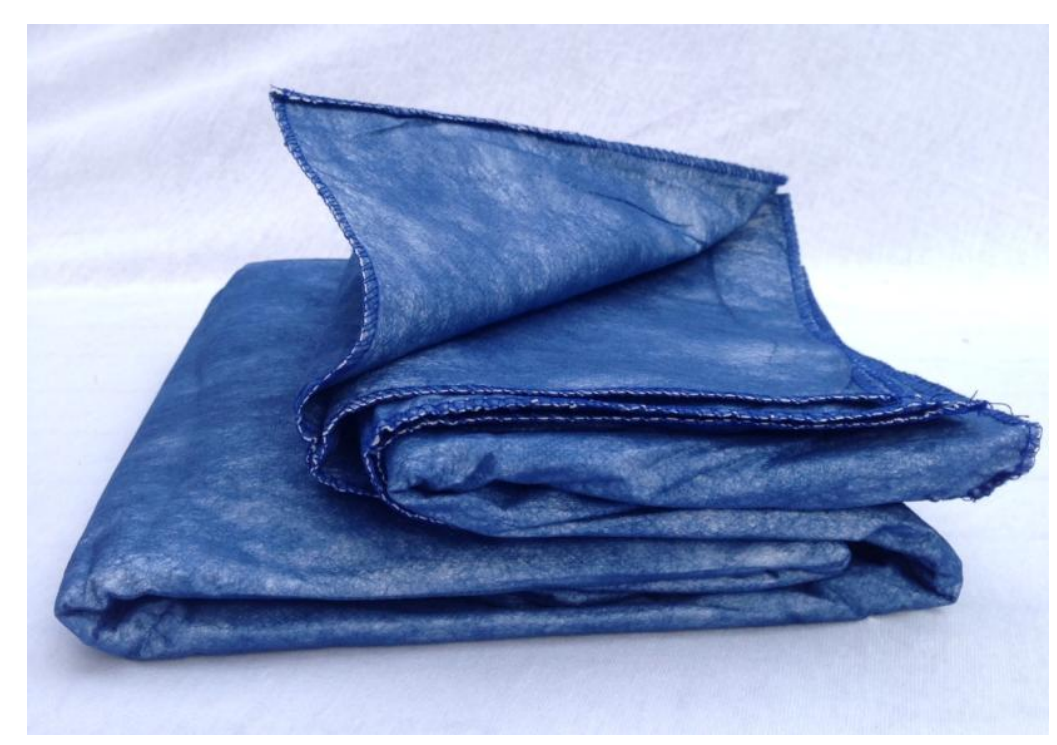

Abbildung 4: Vlies-Einmaldecke zusammengelegt. Quelle: A. Lorey-Tews

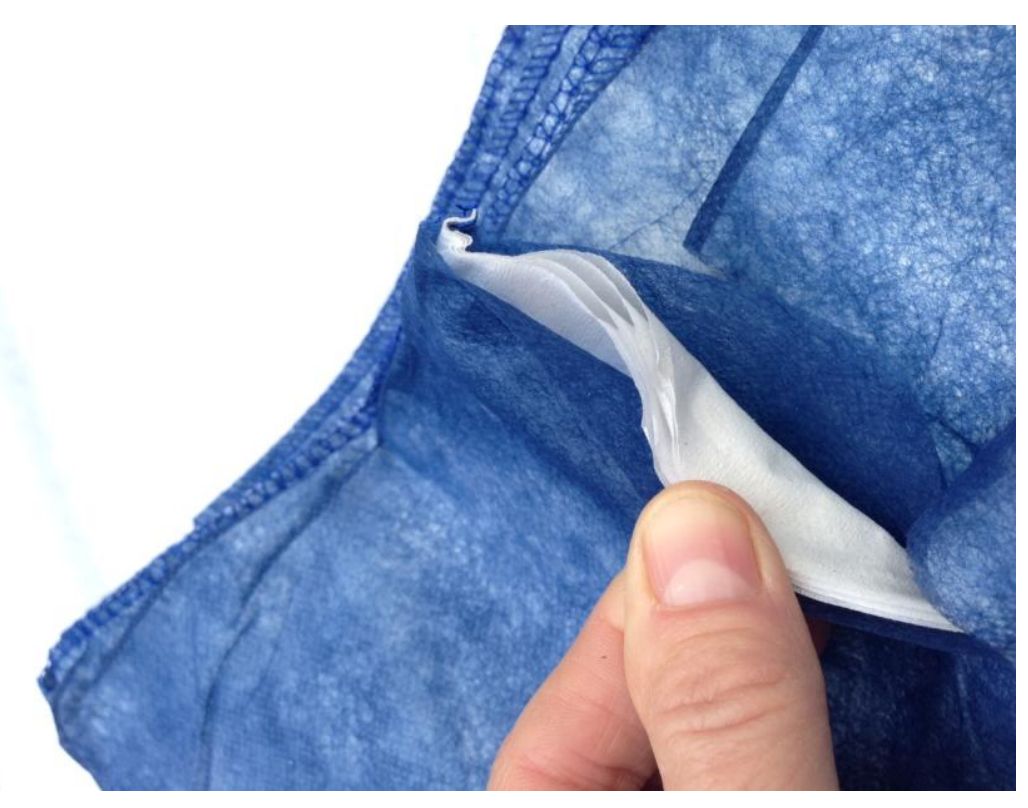

Abbildung 5: Darstellung der Füllung der Vlies-Einmaldecke. Quelle A. Lorey-Tew 


\subsubsection{Probanden-Monitoring}

Die Überwachung der Probanden erfolgte mit einem Multi-Parameter Patientenmonitor Propaq EL 104 EL. Dieses Gerät ist laut Datenblatt des Herstellers für den Einsatz bei den im Kühlhaus herrschenden Umgebungstemperaturen zugelassen.

Folgende Parameter wurden während des gesamten Versuchszeitraums überwacht:

\subsubsection{Herzfrequenz}

Die Überwachung der Herzfrequenz erfolgte kontinuierlich über eine 3-Punkt-Ableitung.

\subsubsection{Blutdruck}

Die Überwachung des Blutdruckes wurde nichtinvasiv über die Brachialarterie am linken Oberarm gemessen. Diese erfolgte oszillometrisch in einem dreiminütigen Intervall, wobei sowohl Systole, Diastole als auch der Mitteldruck bestimmt wurden.

\subsubsection{Periphere Sauerstoffsättigung}

Über eine am linken Zeigefinger angebrachte Pulsoxymetrie wurde kontinuierlich die periphere Sauerstoffsättigung $\left(\mathrm{SpO}_{2}\right)$ der Probanden kontrolliert.

\subsubsection{Messung der Körperkerntemperatur}

Die kontinuierliche Messung der Körperkerntemperatur erfolgte durch eine in den rechten Gehörgang eingebrachte Sonde. Hierfür wurde eine tympanale Sonde der Firma GEHealthcare verwendet. Die Probanden wurden aufgefordert, zwanzig Minuten vor Beginn der Versuche die Sonde im rechten Gehörgang zu platzieren und soweit vorzuschieben, dass ein Kontakt der Sonde mit dem Trommelfell bemerkt wurde. Hiernach wurde der Gehörgang mit Watte abgedeckt und mit einem um den Kopf angelegten Verband sicher fixiert.

\subsubsection{Messung der Hauttemperatur}

Die Hauttemperatur wurde mit dem Infrarot-Thermometer KIRAY 300 bestimmt. An folgenden Hautflächen wurde die Temperatur alle drei Minuten bestimmt:

- Oberarm medial außen rechts 
- Brust

- Oberschenkel medial außen rechts

- Wade medial rechts

Zusätzlich wurde das Kälteempfinden der Probanden dokumentiert. Diese erfolgte mit den folgenden Methoden.

\subsubsection{Numerische Ratingskala}

Die Probanden sollten während der Versuchsreihen anhand einer numerischen Ratingskala

$$
\begin{aligned}
& 1 \text { = angenehmes Wärmegefühl bis } \\
& 10 \text { = maximales Kältegefühl }
\end{aligned}
$$

alle drei Minuten ihr subjektives Kälteempfinden beschreiben.

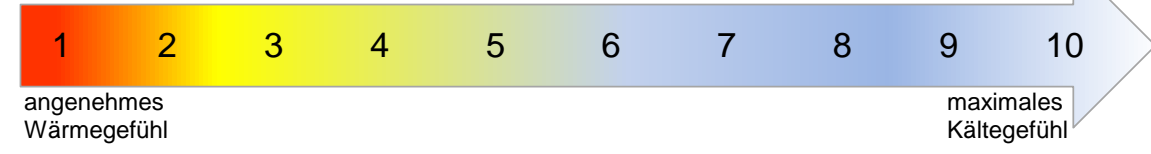

\section{Abbildung 6: Numerische Ratingskala. Quelle: A. Lorey-Tews}

\subsubsection{Subjektive Bewertung der Wärmemethode}

Nach Beendigung des jeweiligen Versuchsschrittes wurde der Proband durch den Prüfarzt nach dessen subjektiver Bewertung der Wärmemethode befragt.

Folgende Antworten gab es dabei zur Auswahl:

- hat überhaupt nichts bewirkt

- hat nur gering gewärmt

- hat mäßig gewärmt

- hat deutlich gewärmt

\subsubsection{Kältezittern}

Mittels einer einfachen, validierten 4-Punkte-Skala wurde durch den Prüfarzt alle drei Minuten die Stärke des Muskelzitterns der Studienteilnehmer qualifiziert. Verwendung fand hierfür der Bedsite-Shivering-Assessment-Scale (BSAS) von Badjatia N. et al. 2008 (Badjatia et al. 2008). 
Tabelle 5 Bedsite- Shivering- Assessment- Scale (BSAS) (Badjatia et al. 2008)

\begin{tabular}{|c|l|l||}
\hline SCORE & TYPE OF SHIVERING & LOCATION \\
\hline $\mathbf{0}$ & None & $\begin{array}{l}\text { No shivering is detected on palpation of the masseter, neck, } \\
\text { or chest muscles }\end{array}$ \\
\hline $\mathbf{1}$ & Mild & Shivering localized to the neck and thorax only \\
\hline $\mathbf{2}$ & Moderate & $\begin{array}{l}\text { Shivering involves gross movement of the upper extremities } \\
\text { (in addition to neck and thorax) }\end{array}$ \\
\hline $\mathbf{3}$ & Severe & $\begin{array}{l}\text { Shivering involves gross movements of the trunk and upper } \\
\text { and lower extremities }\end{array}$ \\
\hline
\end{tabular}

\subsection{Methoden}

\subsubsection{Versuchsdurchführung}

Der grundlegende Versuchsaufbau und zeitliche Ablauf war dem Ablauf eines schweren Verkehrsunfalles mit erforderlicher technischer Rettung und rettungsdienstlicher Versorgung nachempfunden. Die für diese standardisierte Simulation gewählten Zeitabstände orientieren sich hierbei an den, durch die Deutsche Gesellschaft für Unfallchirurgie erhobenen, durchschnittlichen Werten für die durchschnittliche präklinische Versorgungszeit. Diese wird mit $71 \mathrm{~min}(+56 \mathrm{~min}$ ) angegeben (Jahresbericht Traumaregister 2015). Die Zeit für den Transport des Patienten in das Krankenhaus wurde in dem Versuchsaufbau nicht berücksichtigt und daher standardisiert mit einer Zeit von 11 min von der Gesamtversuchszeit abgezogen. Somit ergab sich eine Gesamtzeit des Versuches von $60 \mathrm{~min}$.

Der Versuch gliederte sich in die Versuchsteile A und B. Jeder Proband musste dabei beide Versuchsteile, unterbrochen von mindestens 24 Stunden, durchlaufen. Die Probanden waren bekleidet mit T-Shirt, Shorts, Unterwäsche, Socken und Schuhen. Sie wurden angehalten, vor dem Versuch keinen Kaffee oder heiße Getränke zu konsumieren. 15 Minuten vor Beginn der jeweiligen Versuchsreihe wurde das Patienten- und Temperaturmonitoring angelegt.

Dann wurden die Probanden auf dem PKW-Sitz in der Mitte des Kühlhauses platziert und die Versuchszeit gestartet. Die Probanden waren angehalten, sich während des Versuches möglichst wenig zu bewegen.

Die ersten 21 Minuten waren sowohl in Versuchsteil A als auch Versuchsteil B identisch. Eine Wärmung fand in dieser Zeit nicht statt, die Patienten waren schutzlos den umgebenden Temperaturen ausgeliefert. Dieses sollte die Zeit zwischen Unfallereignis und Beginn der Versorgung am Unfallort simulieren. 


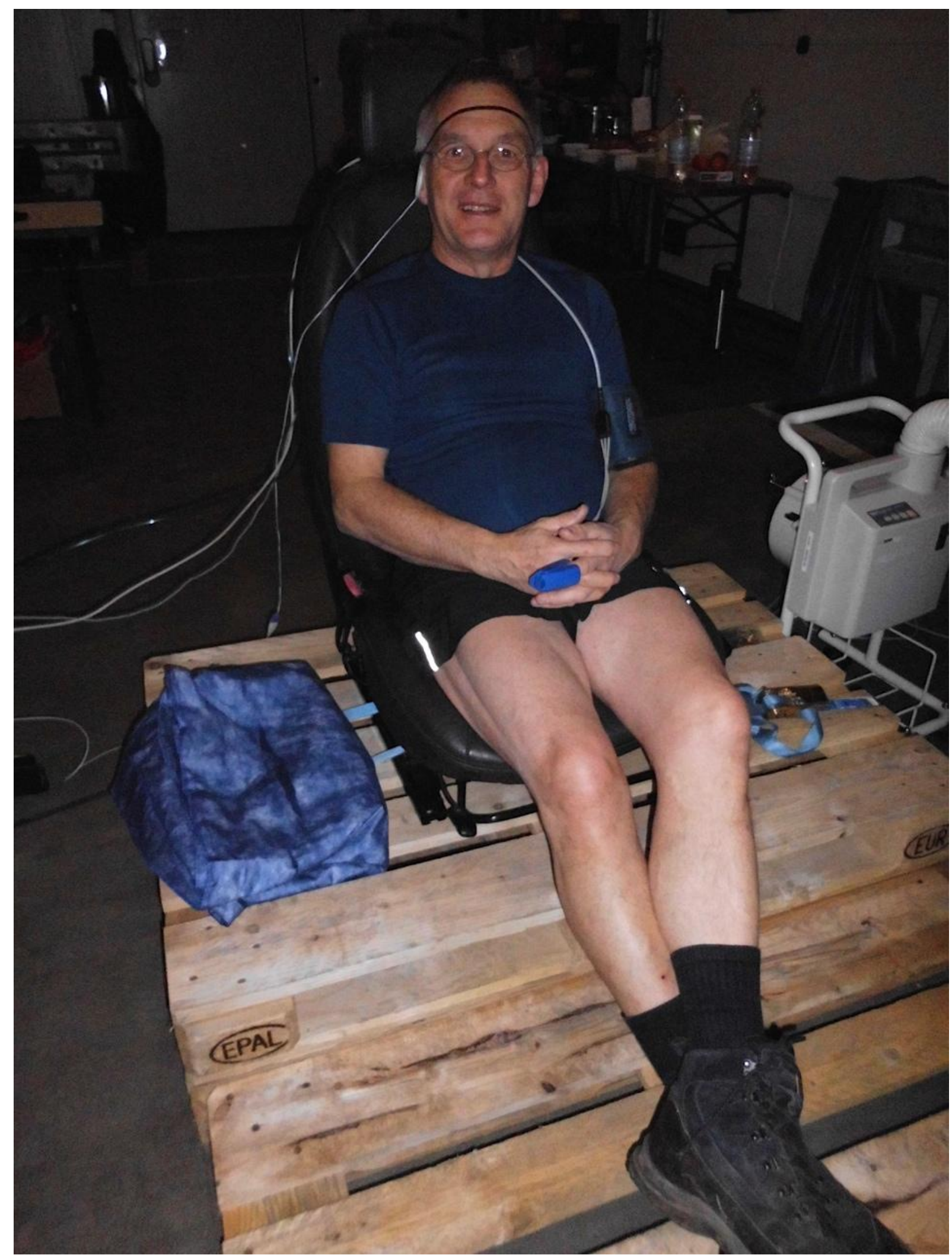

Abbildung 7: Überblick allgemeiner Versuchsaufbau. Quelle: A. Lorey-Tews 


\subsubsection{Randomisierung}

Die Zuteilung der Patienten zu dem Versuchsteil, mit dem sie beginnen mussten, erfolgte über ein Los, welches die Probanden vor Beginn des ersten Versuches ziehen mussten.

Dieses wurde dem Prüfarzt übergeben und erst nach Ablauf von 20 Minuten durch den Prüfarzt geöffnet. Im zweiten Versuchsteil wurde das jeweils andere Wärmeverfahren untersucht.

\subsubsection{Versuchsteil A - konvektiver Luftwärmer}

In Versuchsteil A wurden die Patienten nach 21 Minuten mit einer konvektiven Ganzkörperwärmedecke bedeckt. Der konvektive Luftwärmer wurde gestartet und die Temperaturstufe „Boost“ am Gerät eingestellt. Für die folgenden 39 Minuten wurde der Patient mit dem konvektiven Luftwärmer gewärmt.

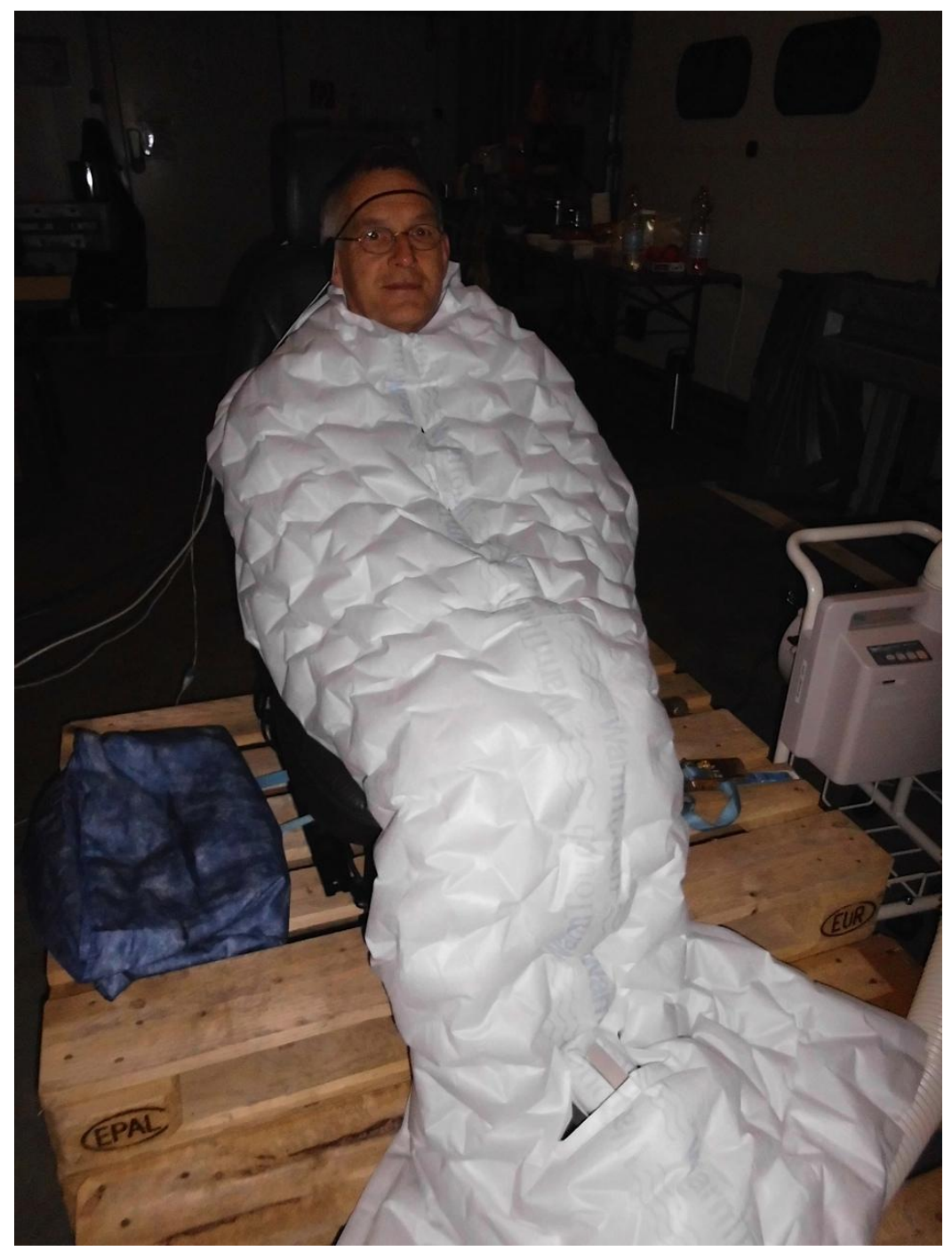

Abbildung 8: Versuchsteil A - konvektiver Luftwärmer. Quelle: A. Lorey-Tews 


\section{Material und Methoden}

\subsubsection{Versuchsteil B - Vlies-Einmaldecke}

Nachdem der Proband 21 Minuten ohne Kälteschutz den Umgebungsbedingungen ausgesetzt war, wurde ihm die Vlies-Einmaldecke um den gesamten Körper, unter Ausschluss des Kopfes, gelegt und zwischen Proband und Autositz festgesteckt. Eingehüllt in der Vliesdecke verblieb der Proband weitere 39 Minuten in seiner bisherigen Position.

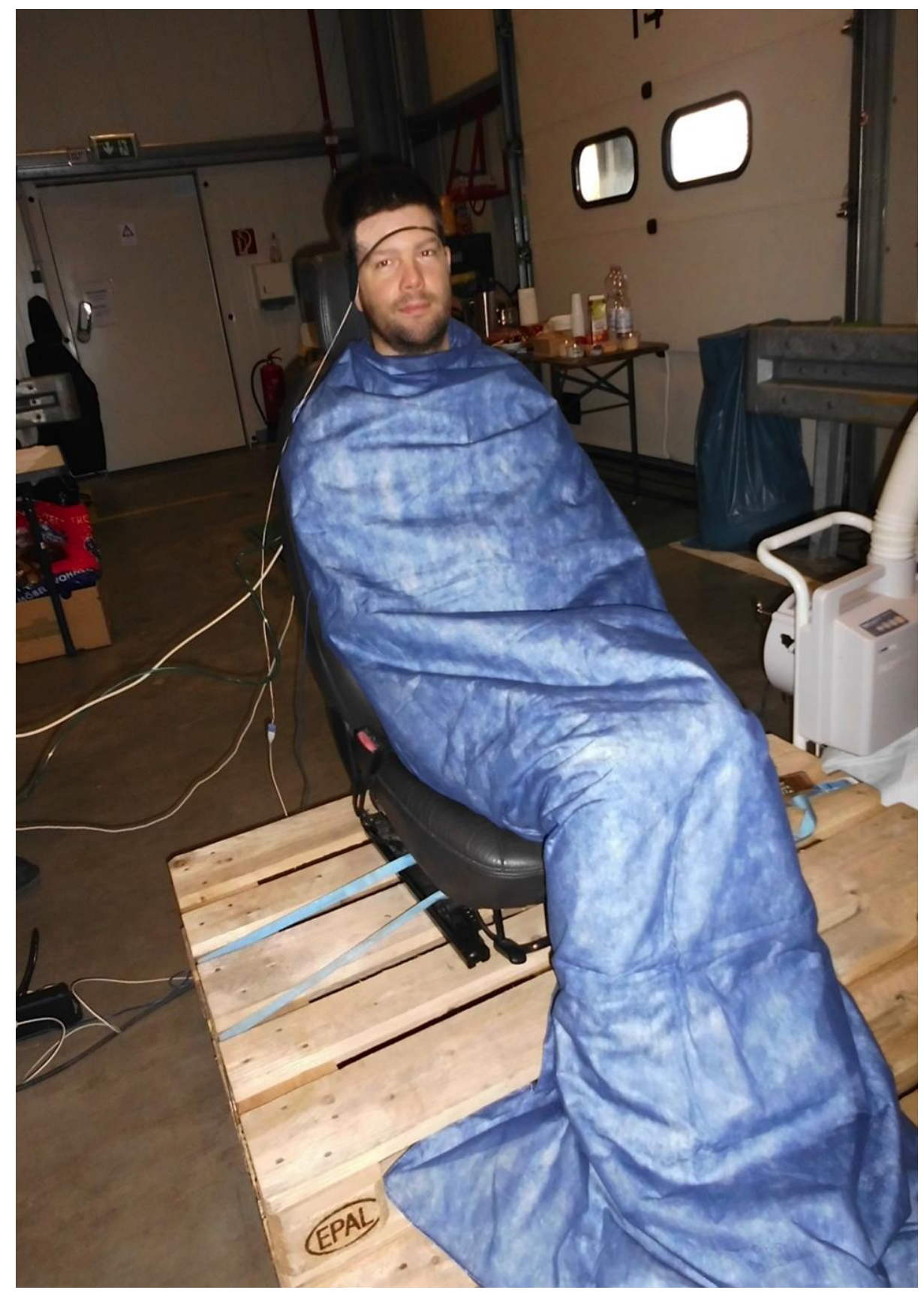

Abbildung 9: Versuchsaufbau B - Vlies-Einmaldecke. Quelle: A. Lorey-Tews 


\subsubsection{Ausgewertete Parameter}

Folgende Parameter wurden alle drei Minuten aufgezeichnet. Der erste Wert wurde zum Startzeitpunkt „0-Minuten“ notiert. Der letzte zum Zeitpunkt „60-Minuten“.

- Herzfrequenz (bpm)

- Systolischer Blutdruck $(\mathrm{mmHg})$

- Mitteldruck (mmHg)

- Diastolischer Blutdruck (mmHg)

- Periphere Sauerstoffsättigung (\%)

- Körperkerntemperatur $\left({ }^{\circ} \mathrm{C}\right)$

- Hauttemperatur Brust, Höhe zweiter Intercostalraum $\left({ }^{\circ} \mathrm{C}\right)$

- Hauttemperatur Oberarm medial $\left({ }^{\circ} \mathrm{C}\right)$

- Hauttemperatur Oberschenkel medial $\left({ }^{\circ} \mathrm{C}\right)$

- Hauttemperatur Wade medial $\left({ }^{\circ} \mathrm{C}\right)$

\subsubsection{Auswertung der gemessenen Temperaturen}

Um den Temperaturverlauf der gemessenen Temperaturen und somit eine statistische Auswertung zu ermöglichen, wurden aus den Messwerten mit Hilfe der angeführten Formeln folgende Parameter berechnet:

\subsubsection{Mittlere Hauttemperatur nach Ramanathan}

Mit Hilfe der 1964 von Ramanathan entwickelten Formel wurde die mittlere Hauttemperatur wie folgt errechnet und ausgewertet (Ramanathan 1964):

$$
\text { MHT }=0,3 \times\left(T_{\text {Brust }}+T_{\text {oberarm }}\right)+0,2 \times\left(T_{\text {Oberschenkel }}+T_{\text {Wade }}\right)
$$

\subsubsection{Mittlere Körpertemperatur}

Die Berechnung der mittleren Körpertemperatur erfolgte mit folgender Formel (Burton 1935):

$$
M K T=\left(064 \times T_{\text {Körperkern }}\right)+(0,36 \times \text { Mittlere Hauttemperatur })
$$




\subsubsection{Gesamtkörperwärmemenge nach Burton}

Aus der Mittleren Körpertemperatur, dem Körpergewicht und der spezifischen Wärmekapazität des menschlichen Körpergewebes wurde die Gesamtkörperwärmemenge aus folgender Formel bestimmt. Die spezifische Wärmekapazität ist eine physikalische Größe, die für einen bestimmten Stoff angibt, welche Wärmemenge, gemessen in der Einheit Joule, diesem Stoff zuzufügen ist, um seine Temperatur um ein Kelvin zu erhöhen.

Die spezifische Wärmekapazität des Menschen beträgt durchschnittlich $3,475 \mathrm{~kJ} \cdot{ }^{\circ} \mathrm{C}^{-1} \cdot \mathrm{kg}^{-1}$ (Burton 1935).

$$
\begin{gathered}
G K W=\text { Mittlere Körpertemperatur }(M K T) \times \text { Gewicht } \times \text { spez.Wärmekapazität } \\
\text { des menschlichen Körpergewebes }\left(3,475 \mathrm{kj}^{\circ} \mathrm{Ckg}^{-1}\right)
\end{gathered}
$$

\subsubsection{Statistische Methoden}

Die statistischen Auswertungen erfolgten mit Hilfe von SPSS für Windows, Version 22.0 (SPSS Inc., U.S.A.). Die Darstellung der metrischen Variablen erfolgte als Mittelwerte und Mediane, während die Streumaße als Standardabweichungen und Quartile angegeben wurden.

Hinsichtlich ihrer Normalverteilung wurden die kontinuierlichen Variablen mittels des Shapiro-Wilk-Tests überprüft. Die getesteten Variablen wiesen überwiegend keine Normalverteilung auf $(p<0,05)$. Bei den Vergleichen der Stichproben wurden daher durchgehend nichtparametrische Tests für nicht normalverteilte Stichproben herangezogen. Mit Hilfe des Wilcoxon-Tests wurden zwei verbundene, nicht normalverteilte Stichproben hinsichtlich signifikanter Unterschiede überprüft, während beim Vergleich von mehr als zwei verbundenen, nicht normalverteilten Stichproben, der Friedman-Test Anwendung fand.

Bei allen durchgeführten Tests erfolgte eine zweiseitige Signifikanzüberprüfung. Wobei für alle statistischen Tests ein $\mathrm{p}$-Wert $<0,05$ als statistisch signifikant angenommen wurde.

Auch die Grafiken wurden mit SPSS erstellt. Zur Darstellung der Mediane und Quartilsabstände wurden Boxplots angelegt. In den Boxen sind der Median und die 25.-75. Perzentile aufgetragen. Die T-Balken entsprechen dem kleinsten und größten Wert. Ausreißer sind die Werte, die zwischen 1 1/2 - 3 Boxlängen außerhalb der Box lagen. In den Grafiken sind sie als Kreise dargestellt, während Extremwerte, welche mehr als drei Boxlängen außerhalb der Box gemessen wurden, als Kreuze aufgetragen sind.

Die Altersverteilung wurde in einem Histogramm dargestellt. 


\section{Ergebnisse}

\subsection{Studienflowchart}
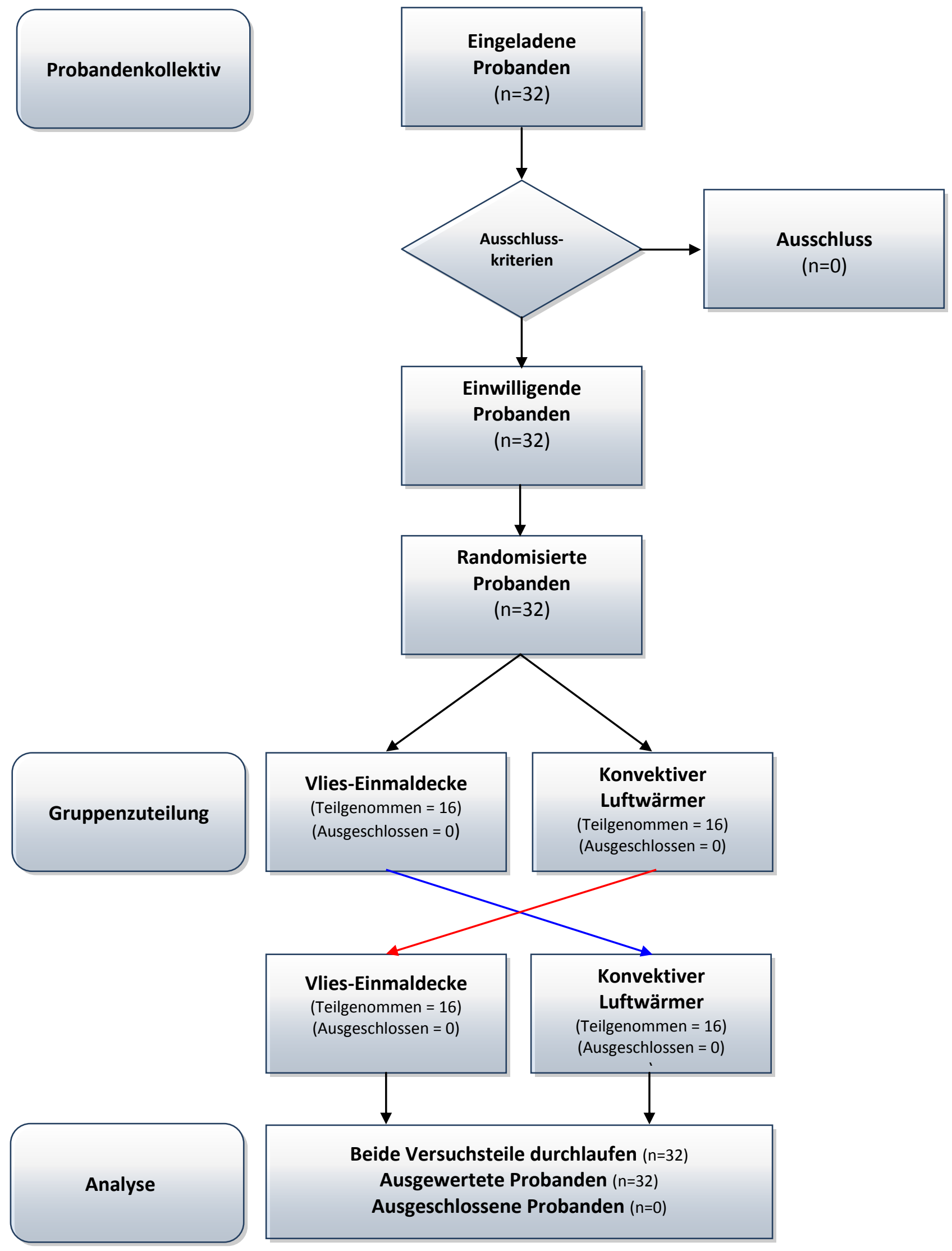


\subsection{Probandensicherheit}

Zu keiner Zeit der Versuchsabläufe traten Ereignisse auf oder wurden Messwerte erhoben, welche die körperliche Unversehrtheit der Probanden gefährdeten. Die gemessenen Hauttemperaturen lagen zu keiner Zeit über $43{ }^{\circ} \mathrm{C}$, oder unter $35{ }^{\circ} \mathrm{C}$. Die unter 2.2.1 genannten Vorgaben der Ethikkommission wurden jederzeit eingehalten und beachtet.

\subsection{Basisparameter}

Insgesamt durchliefen 32 Probanden den kompletten Versuchsablauf.

Von den teilnehmenden Probanden waren 56,3\% Männer und 43,7\% Frauen. Dieses entspricht in der Gewichtung den Daten der Deutschen Gesellschaft für Unfallchirurgie. Auch dort ist der Anteil der Männer unter den schwerverletzten Patienten höher, als der Anteil der Frauen. Das Alter betrug im Median 45,2 Jahre und bildete damit ebenfalls das Patientenkollektiv der Schwerverletzten in der o.g. Datenbank nährungsweise ab. Dort beträgt das Alter der Schwerverletzten gemittelt über die letzten 10 Jahre 48,6 Jahre (Jahresbericht Traumaregister 2015).

Die Körpergröße des Patientenkollektivs beträg im Median $179 \mathrm{~cm}$. Das Körpergewicht bezogen auf den Body-Mass-Index beträgt 25,8.

Tabelle 6 Geschlechterverteilung

\begin{tabular}{|l|r|r|}
\hline & Häufigkeit & Prozent \\
\hline männlich & 18 & 56,3 \\
weiblich & 14 & 43,8 \\
Gesamtsumme & 32 & 100,0 \\
\hline
\end{tabular}

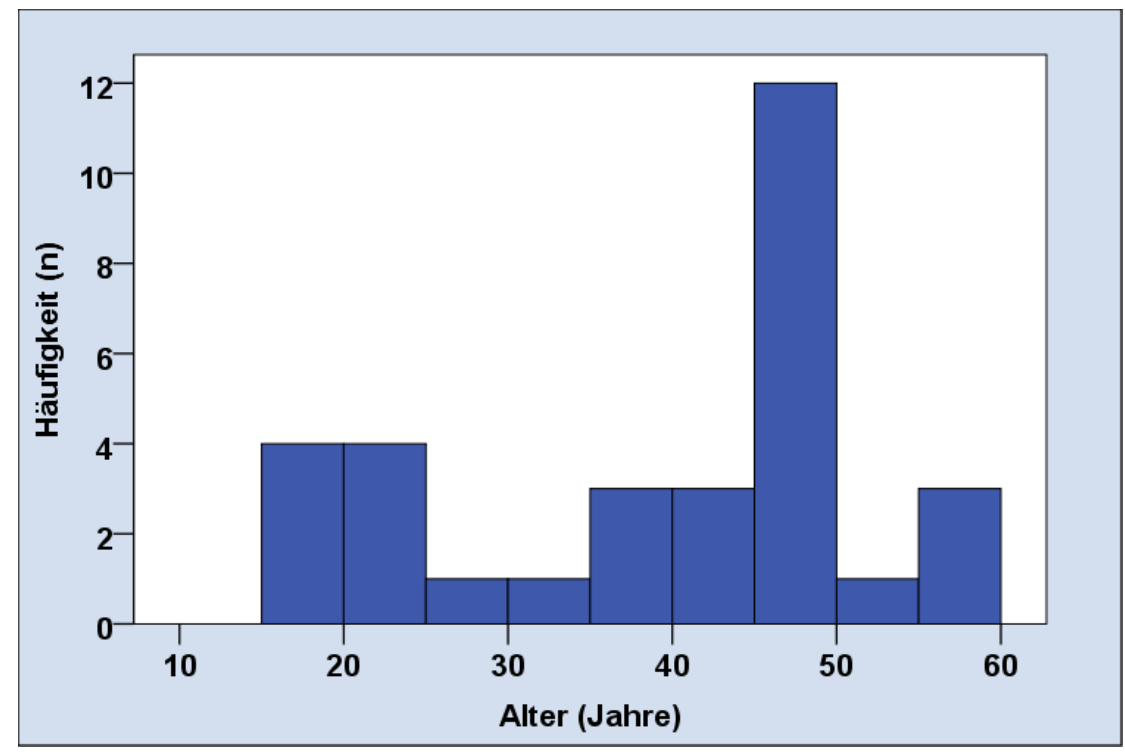

Abbildung 10: Histogramm Altersverteilung der Probanden 
Tabelle 7 Größe und Gewicht

\begin{tabular}{|ll|r|r|r|}
\hline & & Größe & Gewicht & \multicolumn{1}{|c|}{ BMI } \\
\hline N & Gültig & 32 & 32 & 32 \\
& Fehlend & 0 & 0 & 0 \\
Median & & 179,00 & 79,00 & 25,8078 \\
Minimum & & 158 & 51 & 17,24 \\
Maximum & & 196 & 115 & 29,24 \\
Perzentile & 25 & 170,25 & 66,25 & 22,0589 \\
& 75 & 188,75 & 91,00 & 27,0156 \\
\hline
\end{tabular}

\subsection{Ergebnisse Vitalparameter}

Im Folgenden sind die Ergebnisse der erhobenen Vitalparameter „Herzfrequenz“, „Blutdruck“ und „Sauerstoffsättigung“ dargestellt.

\subsubsection{Herzfrequenz (HF)}

Bei der Herzfrequenz ergaben sich zu den Versuchszeitpunkten 0 Minuten und 21 Minuten keine signifikanten Unterschiede in der statistischen Auswertung. Dort betrugen die im Versuchsteil „konvektiver Luftwärmer" im Median gemessenen Frequenzen 71,5/min (Zeitpunkt 0 Min) und 71,0/min (Zeitpunkt 21 Min). Im Versuchsteil „Vliesdecke“ betrugen die Mediane 74,0/min (Zeitpunkt 0 Min) und 74,5/min (Zeitpunkt 21 Min). Auch zu den Zeitpunkten 45 Minuten (70,5/min „Luftwärmer“ vs. 71,5/min „Vliesdecke“) und 60 Minuten (70,5/min „Luftwärmer" und 73,5/min „Vliesdecke“) ergaben sich keine signifikanten Differenzen:

Lediglich zum Zeitpunkt 30 Minuten konnte ein signifikanter Unterschied nach gewiesen werden. Die Herzfrequenz betrug im Median, zum Zeitpunkt 30 Minuten im Versuchsteil A „konvektiver Luftwärmer“ 68,5/min, während der Median im Versuchsteil B „VliesEinmaldecke“ 76,5/min betrug. 


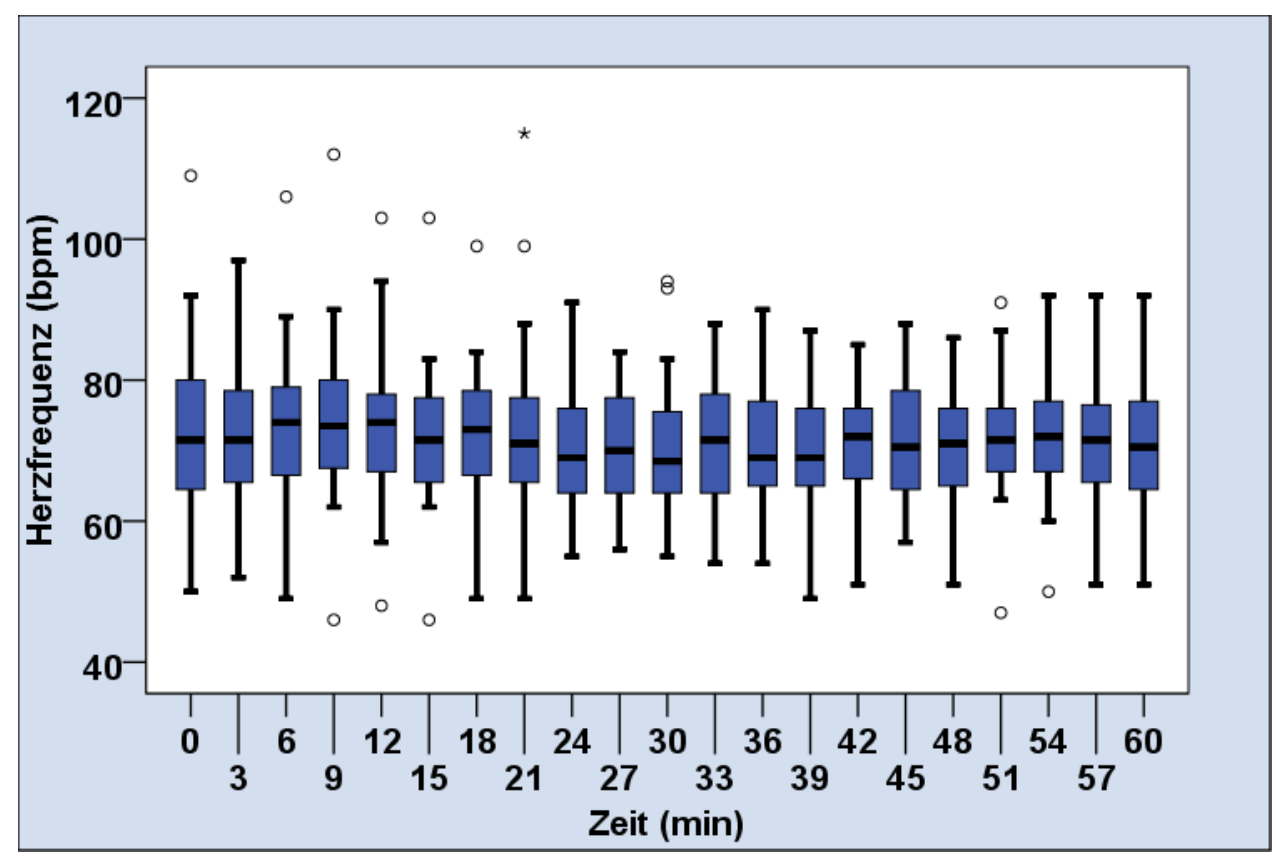

Abbildung 11: Herzfrequenz im Versuchsteil mit konvektiver Luftwärmung

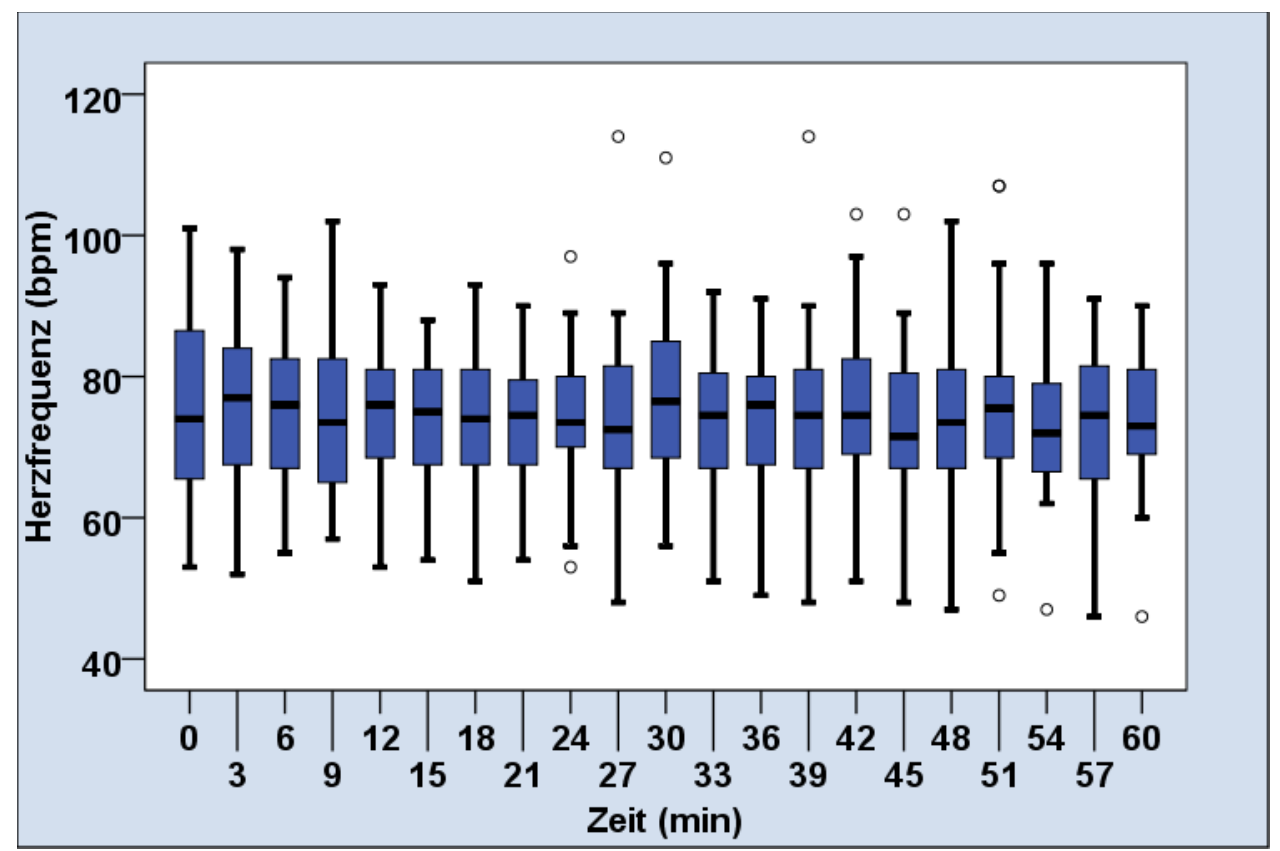

Abbildung 12 Herzfrequenz im Versuchteil mit Vliesdecke

Tabelle 8 Wilcoxon-Test Herzfrequenz Luftwärmer / Vliesdecke

\begin{tabular}{|l|r|r|r|r|r|}
\hline & $\begin{array}{l}\text { HF T0 - } \\
\text { HF T0 }\end{array}$ & $\begin{array}{c}\text { HF T21 - HF } \\
\text { T21 }\end{array}$ & $\begin{array}{c}\text { HF T30 - HF } \\
\text { T30 }\end{array}$ & $\begin{array}{r}\text { HF T45 - HF } \\
\text { T45 }\end{array}$ & $\begin{array}{c}\text { HF T60 - HF } \\
\text { T60 }\end{array}$ \\
\hline $\begin{array}{l}\text { Asymptotische Signifikanz } \\
\text { (2-seitig) }\end{array}$ & $\mathbf{1 2 0}$ & $\mathbf{1 6 4}$ & $\mathbf{0 0 6}$ &, $\mathbf{9 1 8}$ &, $\mathbf{1 6 0}$ \\
\hline
\end{tabular}




\subsubsection{Blutdruck (RR)}

Die Blutdruckwerte wurden unterteilt in systolischer Blutdruck, diastolischer Blutdruck und Mitteldruck getrennt statistisch ausgewertet.

\subsubsection{Systolischer Blutdruck (RR)}

Die Auswertung des systolischen Blutdruckes erbrachte keine signifikanten Unterschiede zu den Zeitpunkten 21 und 45 Minuten.

Lediglich zu den Zeitpunkten 30 Minuten und 60 Minuten konnten grenzwertige signifikante Unterschiede gemessen werden. So war der systolische Blutdruck im Versuchsteil A „Luftwärmer" im Median geringer im Vergleich zu dem in Versuchsteil B „Vliesdecke“ gemessenen. Der systolische Blutdruck betrug zum Zeitpunkt 30 Minuten $128 \mathrm{mmHg}$ bzw. 131,5 mmHg (Versuchsteil A / Versuchsteil B) und zum Zeitpunkt 60 Minuten 130,5 mmHg bzw. $133,5 \mathrm{mmHg}$.

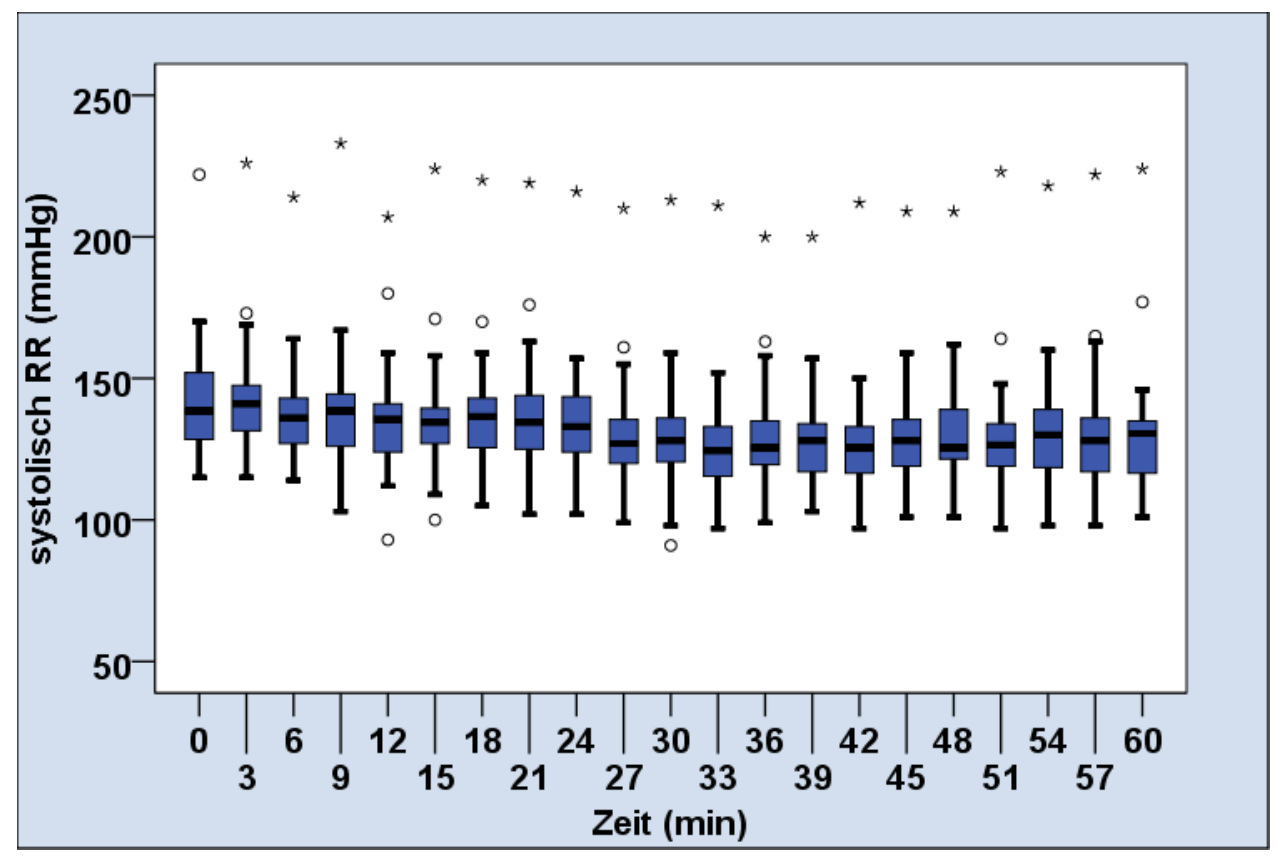

Abbildung 13 systolischer Blutdruck im Versuchsteil mit konvektiver Luftwärmung 


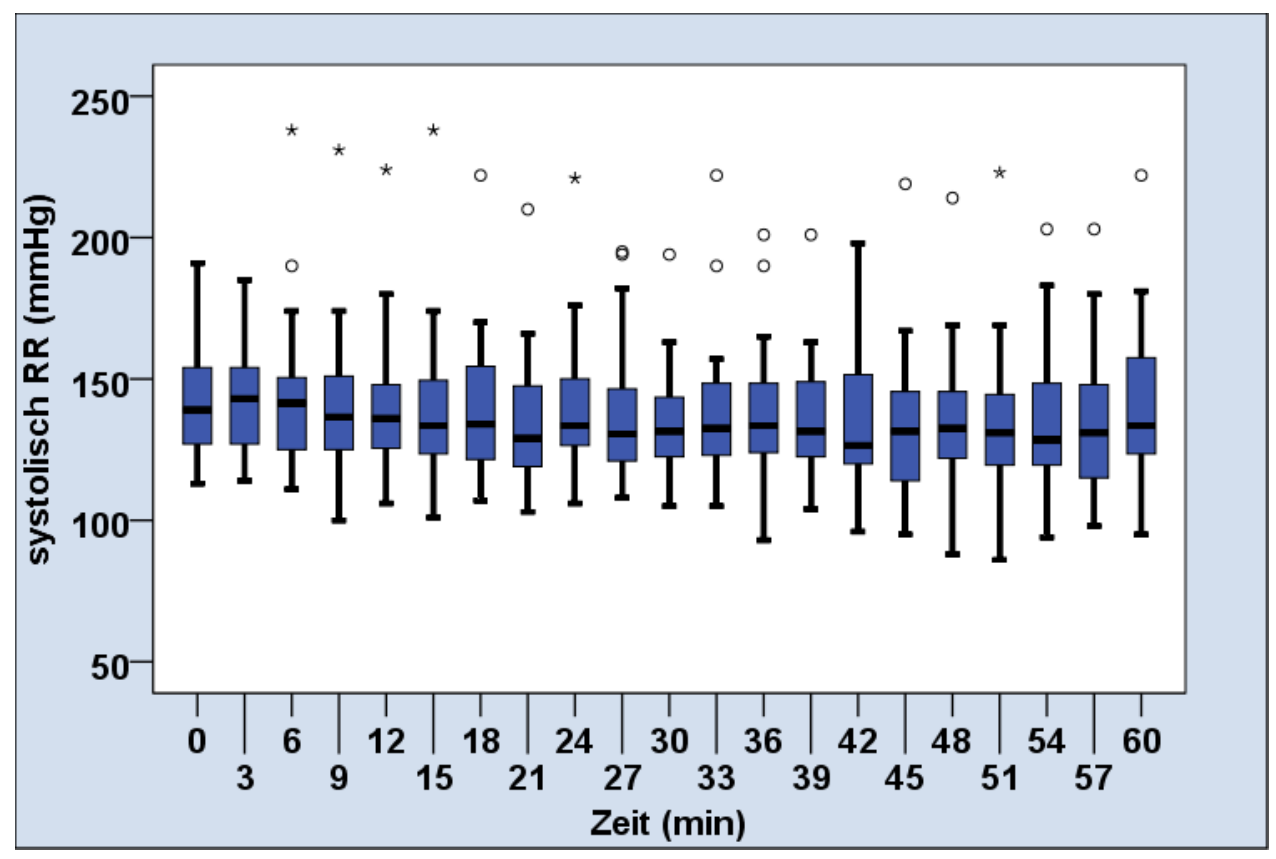

Abbildung 14 systolischer Blutdruck im Versuchteil mit Vliesdecke

Tabelle 9 Wilcoxon-Test systolischer Blutdruck Luftwärmer / Vliesdecke

\begin{tabular}{|l|c|r|r|r|r|}
\hline & $\begin{array}{c}\text { systolisch RR T0 } \\
\text { - systolisch RR } \\
\text { T0 }\end{array}$ & $\begin{array}{c}\text { systolisch RR } \\
\text { T21 - systolisch } \\
\text { RR T21 }\end{array}$ & $\begin{array}{c}\text { systolisch RR } \\
\text { T30 - systolisch } \\
\text { RR T30 }\end{array}$ & $\begin{array}{c}\text { systolisch RR } \\
\text { T45 - systolisch } \\
\text { RR T45 }\end{array}$ & $\begin{array}{c}\text { systolisch RR } \\
\text { T60 - systolisch } \\
\text { RR T60 }\end{array}$ \\
\hline $\begin{array}{l}\text { Asymptotische Signifikanz } \\
\text { (2-seitig) }\end{array}$ & $\mathbf{8 6 8}$ & $\mathbf{4 6 8}$ &, 064 &, $\mathbf{1 5 5}$ &, 075 \\
\hline
\end{tabular}

\subsubsection{Diastolischer Blutdruck (RR)}

Der Wilcoxon-Test zeigte bei der Auswertung des diastolischen Blutdrucks lediglich einen signifikanten Unterschied zum Zeitpunkt 60 Minuten. Der diastolische Blutdruck war im Versuchsteil A „Luftwärmer" signifikant niedriger im Vergleich zum Versuchsteil B „Vliesdecke“ und betrug im Median $81,55 \mathrm{mmHg}$ im Versuchsteil A und $87 \mathrm{mmHg}$ im Versuchsteil B. 


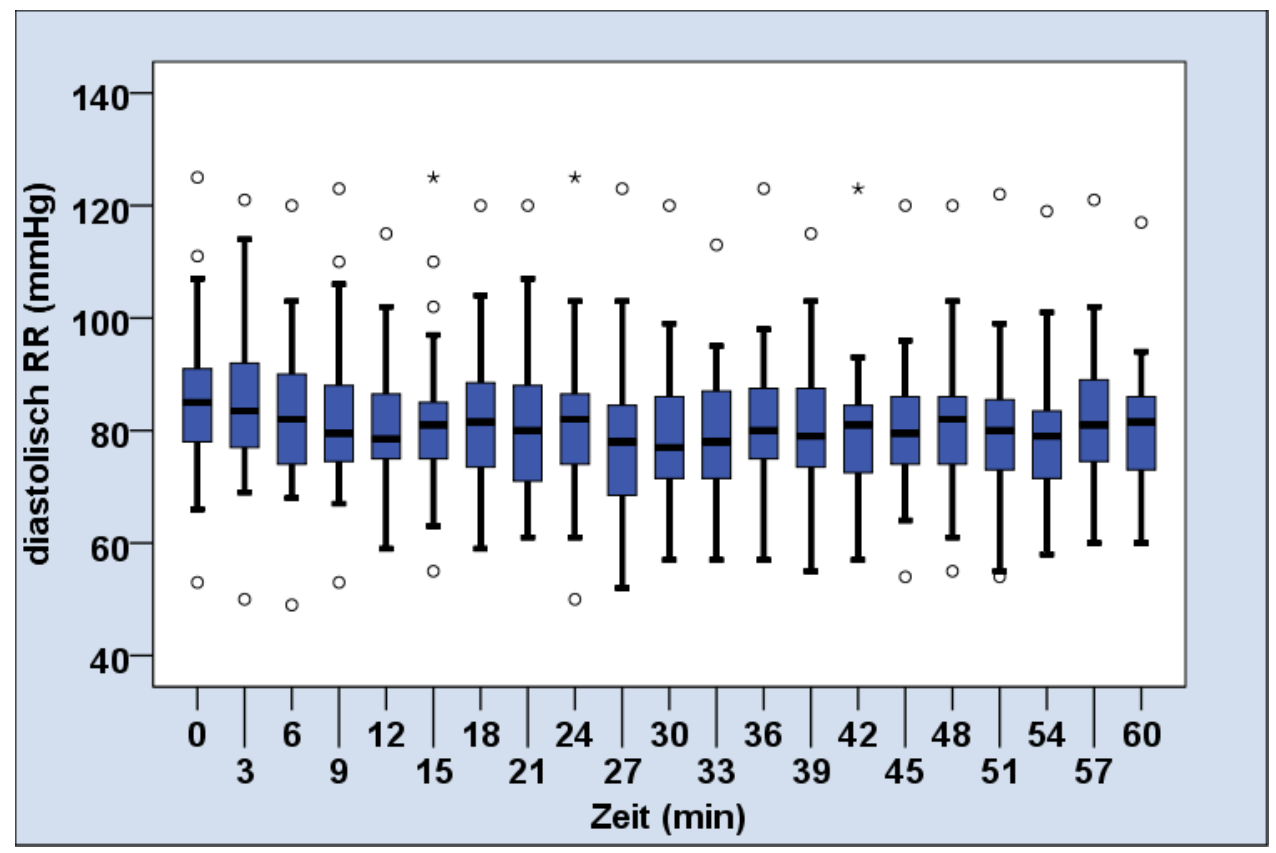

Abbildung 15: diastolischer Blutdruck im Versuchsteil mit konvektiver Luftwärmung

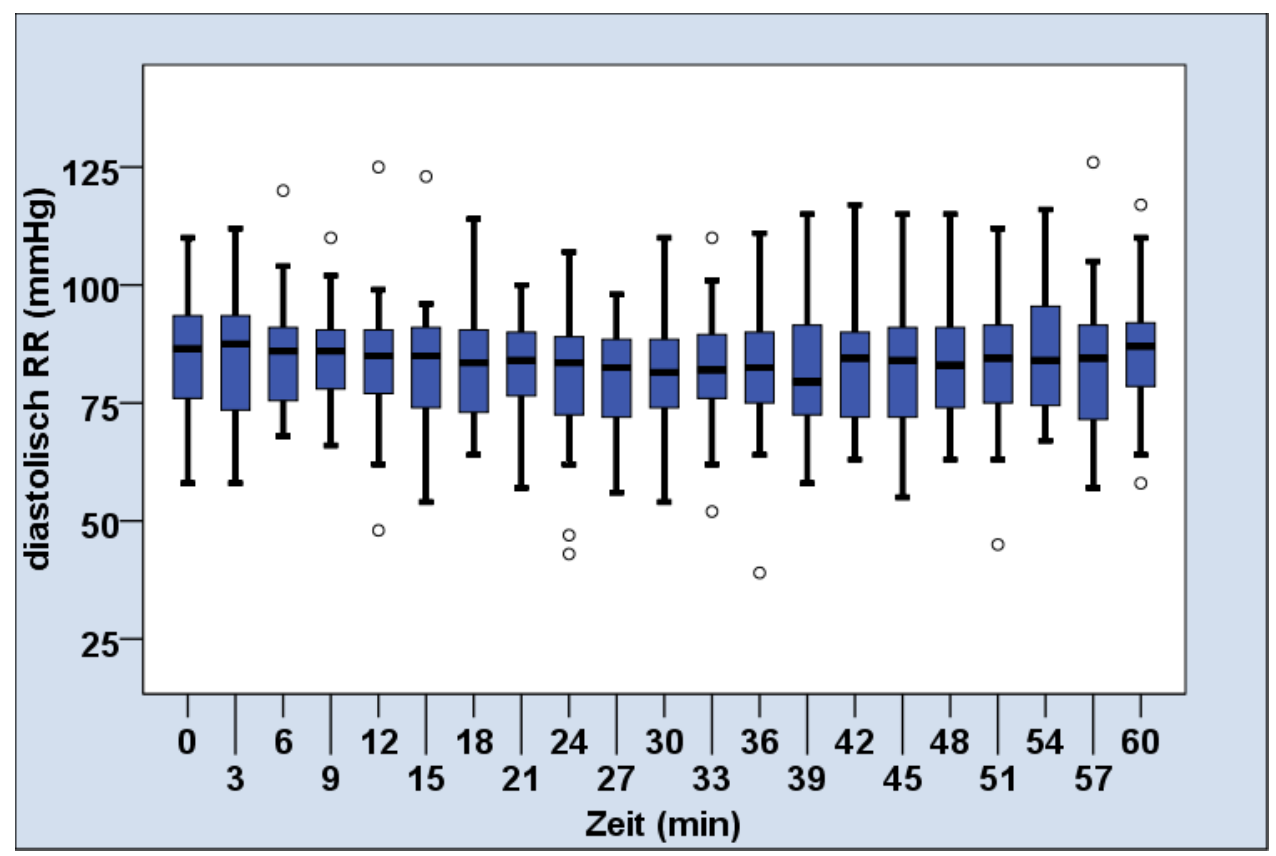

Abbildung 16: diastolischer Blutdruck im Versuchteil mit Vliesdecke

Tabelle 10: Wilcoxon-Test diastolischer Blutdruck Luftwärmer / Vliesdecke

\begin{tabular}{|c|c|c|c|c|c|}
\hline & $\begin{array}{c}\text { diastolisch RR } \\
\text { TO - diastolisch } \\
\text { RR TO }\end{array}$ & $\begin{array}{c}\text { diastolisch RR } \\
\text { T21 - } \\
\text { diastolisch RR } \\
\text { T21 }\end{array}$ & $\begin{array}{c}\text { diastolisch RR } \\
\text { T30 - } \\
\text { diastolisch RR } \\
\text { T30 }\end{array}$ & $\begin{array}{c}\text { diastolisch RR } \\
\text { T45 - } \\
\text { diastolisch RR } \\
\text { T45 }\end{array}$ & $\begin{array}{c}\text { diastolisch RR } \\
\text { T60 - } \\
\text { diastolisch RR } \\
\text { T60 }\end{array}$ \\
\hline $\begin{array}{l}\text { Asymptotische Signifikanz } \\
\text { (2-seitig) }\end{array}$ & 791 & ,511 & ,524 & 279, & 014 \\
\hline
\end{tabular}




\subsubsection{Mitteldruck (RR)}

Beim Mitteldruck zeigte sich in der Auswertung zum Zeitpunkt 21 Minuten ein grenzwertig signifikanter Unterschied und zum Zeitpunkt 60 Minuten ein signifikanter Unterschied zwischen den beiden Versuchsreihen. Im Median sind die Werte des Mitteldrucks im Warmluftversuch niedriger als bei der Wärmung mittels Vliesdecke. So betrugen die MedianWerte zum Zeitpunkt 21 Minuten 94,5 mmHg bzw. 98,0 mmHg (Warmluft bzw. Vliesdecke) und zum Zeitpunkt 60 Minuten 96,0 mmHg bzw. 100,0 mmHg (Warmluft bzw. Vliesdecke).

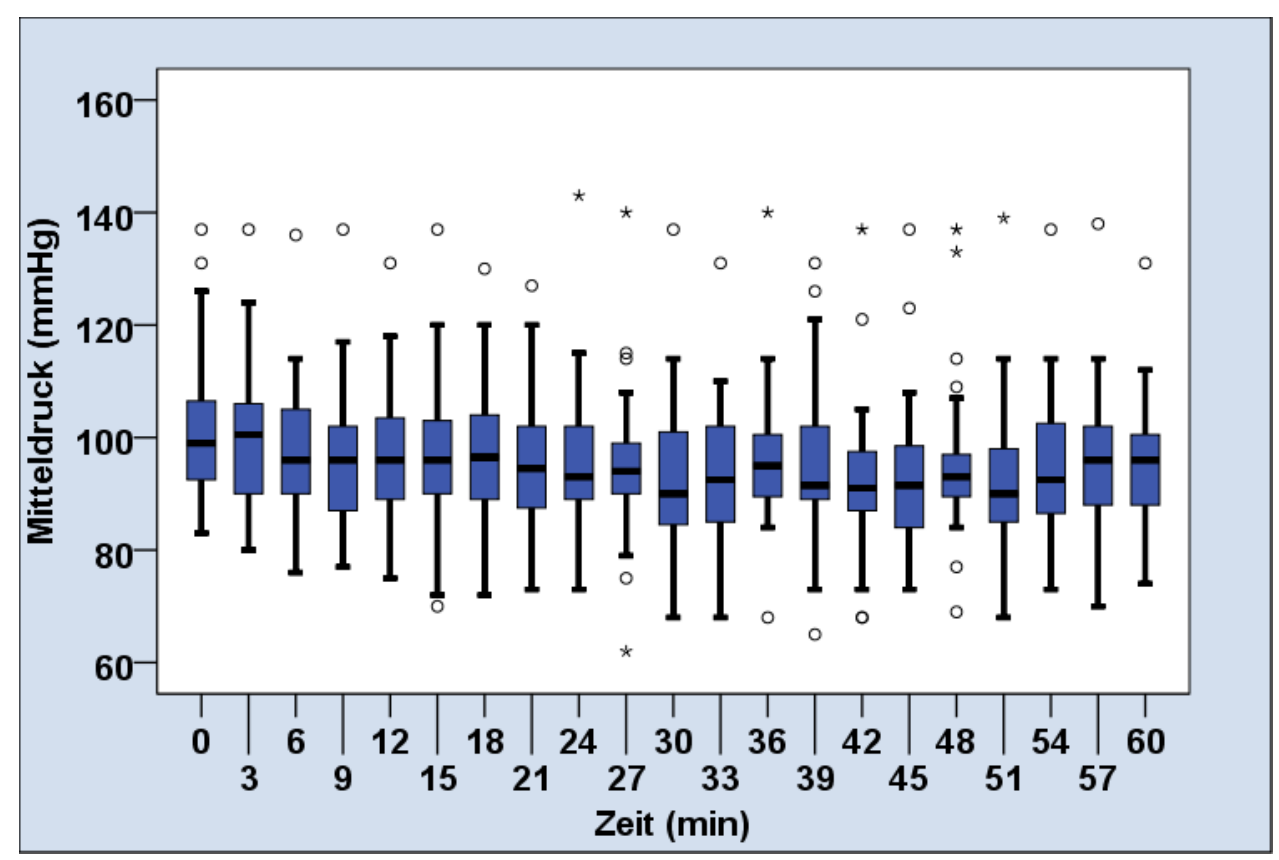

Abbildung 17: Mitteldruck im Versuchsteil mit konvektiver Luftwärmung

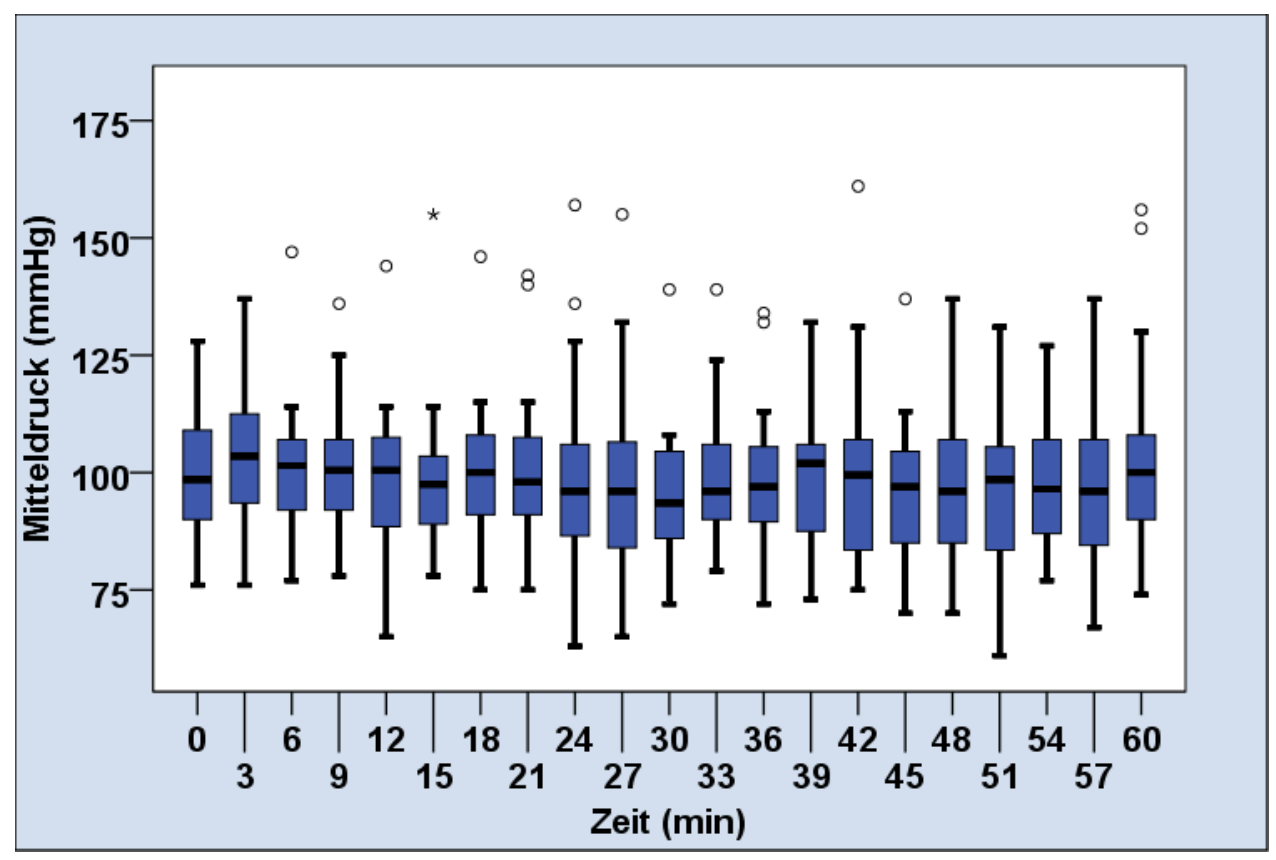

Abbildung 18: Mitteldruck im Versuchteil mit Vliesdecke 


\section{Ergebnisse}

Tabelle 11: Wilcoxon-Test Mitteldruck Luftwärmer / Vliesdecke

\begin{tabular}{|l|r|r|r|r|r|}
\hline & $\begin{array}{c}\text { Mitteldruck T0 - } \\
\text { Mitteldruck T0 }\end{array}$ & $\begin{array}{c}\text { Mitteldruck T21 } \\
- \text { Mitteldruck } \\
\text { T21 }\end{array}$ & $\begin{array}{c}\text { Mitteldruck T30 } \\
- \text { Mitteldruck } \\
\text { T30 }\end{array}$ & $\begin{array}{c}\text { Mitteldruck T45 } \\
\text { - Mitteldruck } \\
\text { T45 }\end{array}$ & $\begin{array}{c}\text { Mitteldruck T60 } \\
- \text { Mitteldruck } \\
\text { T60 }\end{array}$ \\
\hline $\begin{array}{l}\text { Asymptotische Signifikanz } \\
\text { (2-seitig) }\end{array}$ &, 658 &, 065 &, 259 &, 357 &, 015 \\
\hline
\end{tabular}

\subsubsection{Periphere Sauerstoffsättigung $\left(\mathrm{SpO}_{2}\right)$}

Zum Zeitpunkt 60 Minuten zeigte sich bei der Auswertung der peripheren Sauerstoffsättigung mittels Wilcoxon-Test ein signifikanter Unterschied. Die errechneten Mediane betrugen im Versuchsteil A „Luftwärmer“ zum Zeitpunkt 60 Minuten 98,0\% und im Versuchsteil B „Vliesdecke“ 98,5\%.

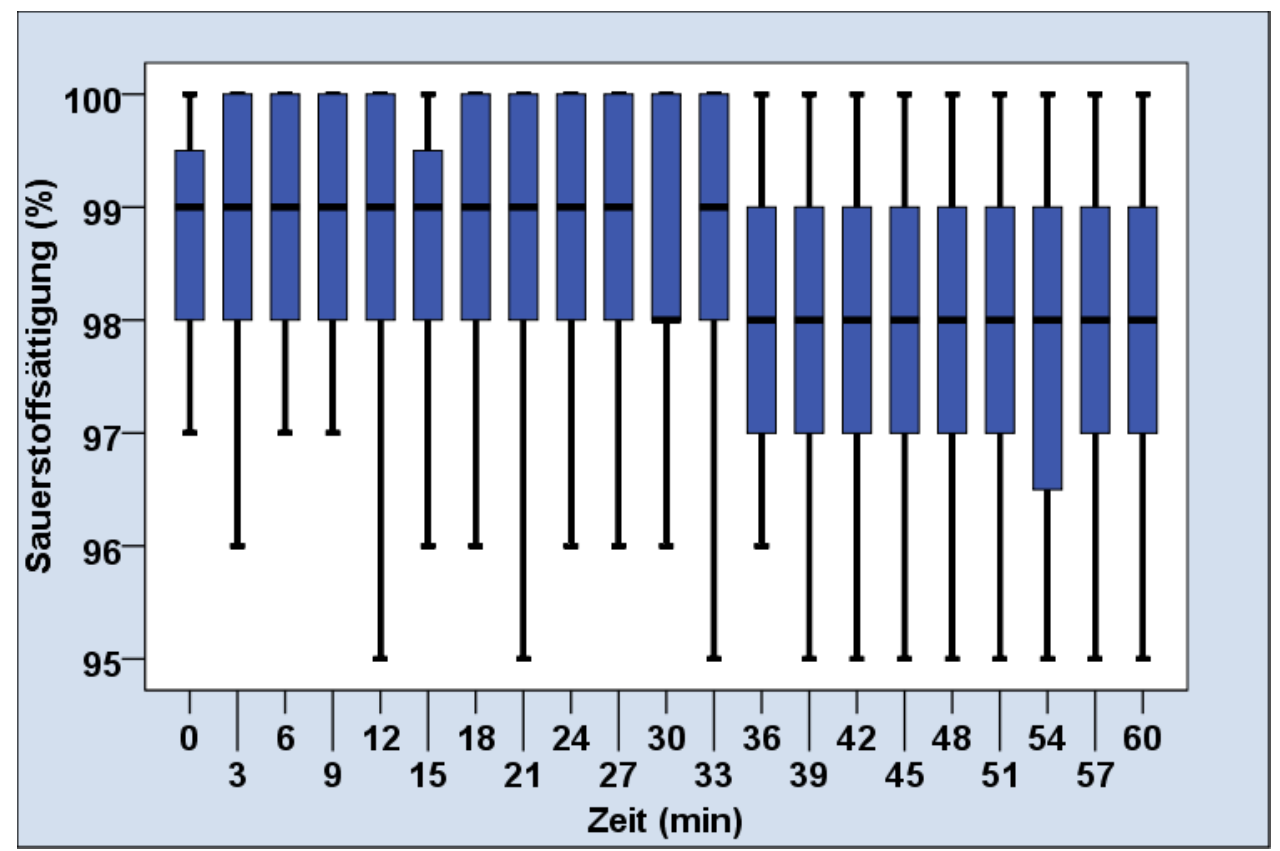

Abbildung 19: periphere Sauerstoffsättigung im Versuchsteil mit konvektiver Luftwärmung 


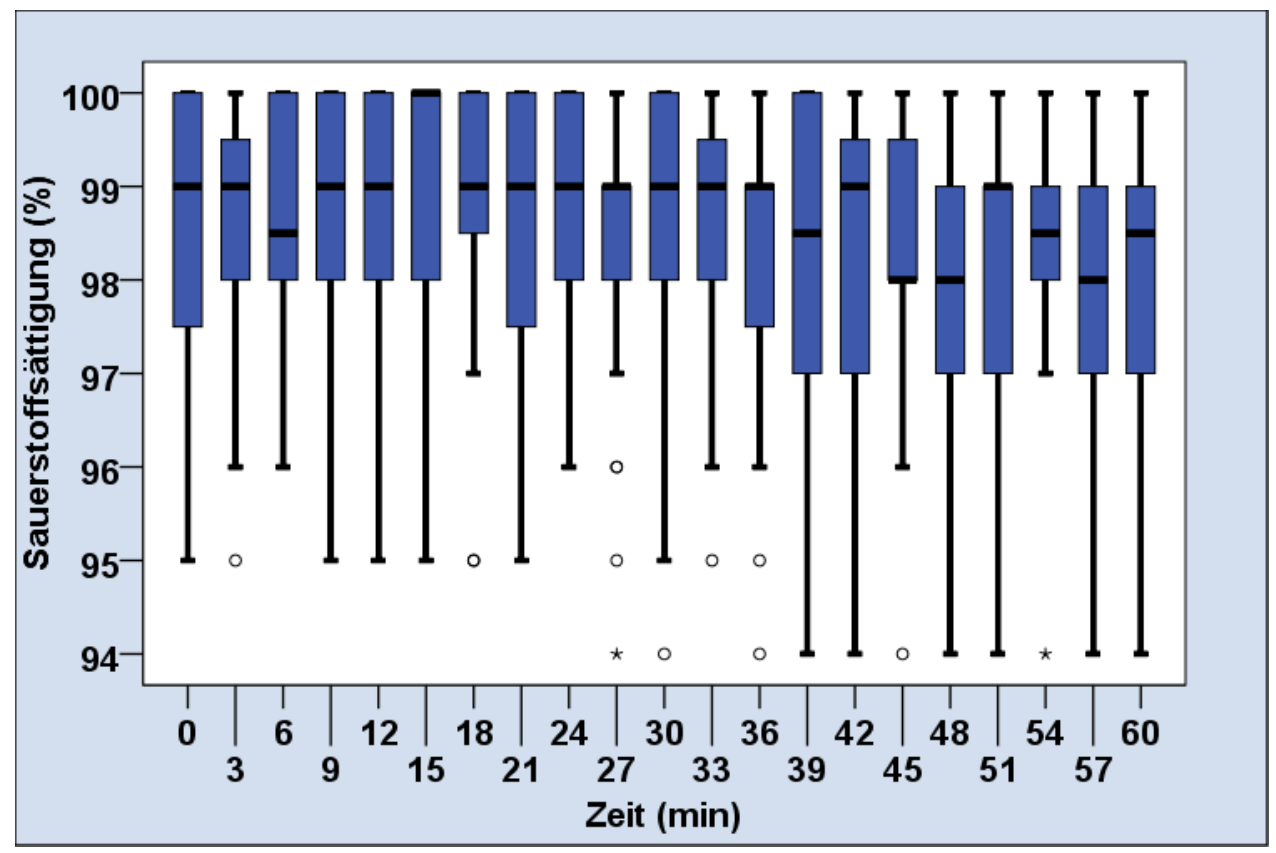

Abbildung 20: periphere Sauerstoffsättigung im Versuchteil mit Vliesdecke

Tabelle 12: Wilcoxon-Test periphere Sauerstoffsättigung Luftwärmer / Vliesdecke

\begin{tabular}{|l|r|r|r|r|r|}
\hline & $\begin{array}{c}\text { Sp T0 - } \\
\text { Sp T0 }\end{array}$ & $\begin{array}{c}\text { Sp T21 - } \\
\text { Sp T21 }\end{array}$ & $\begin{array}{c}\text { Sp T30 - } \\
\text { Sp T30 }\end{array}$ & $\begin{array}{c}\text { Sp T45 - } \\
\text { Sp T45 }\end{array}$ & $\begin{array}{r}\text { Sp T60 - } \\
\text { Sp T60 }\end{array}$ \\
\hline $\begin{array}{l}\text { Asymptotische Signifikanz } \\
\text { (2-seitig) }\end{array}$ & $\mathbf{8 8 1}$ &, $\mathbf{7 0 8}$ &, $\mathbf{8 3 5}$ &, $\mathbf{2 5 2}$ \\
\hline
\end{tabular}

\subsection{Ergebnisse Temperaturmessungen}

\subsubsection{Körperkerntemperatur (KT)}

Bei der statistischen Auswertung der Körperkerntemperatur konnten zu keinem Zeitpunkt signifikante Unterschiede nachgewiesen werden. Betrachtet man die Mediane, so zeigt sich in den beiden Versuchsteilen nach dem Beginn des Versuches ein leichter Anstieg der Körperkerntemperatur bis hin zur 21 Minute. Danach verändert sich die Körperkerntemperatur nur noch unwesentlich, sie stabilisiert sich. 


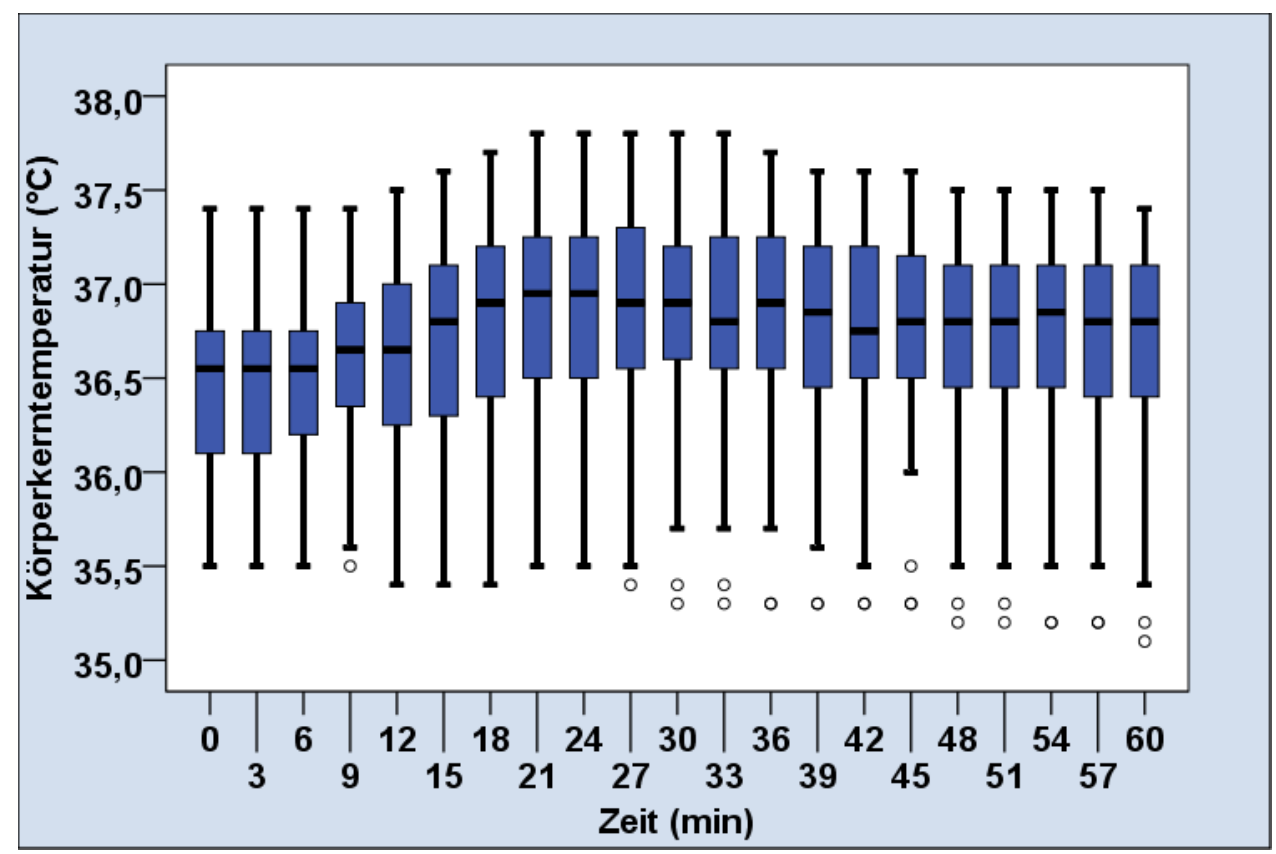

Abbildung 21: Körperkerntemperatur im Versuchsteil mit konvektiver Luftwärmung

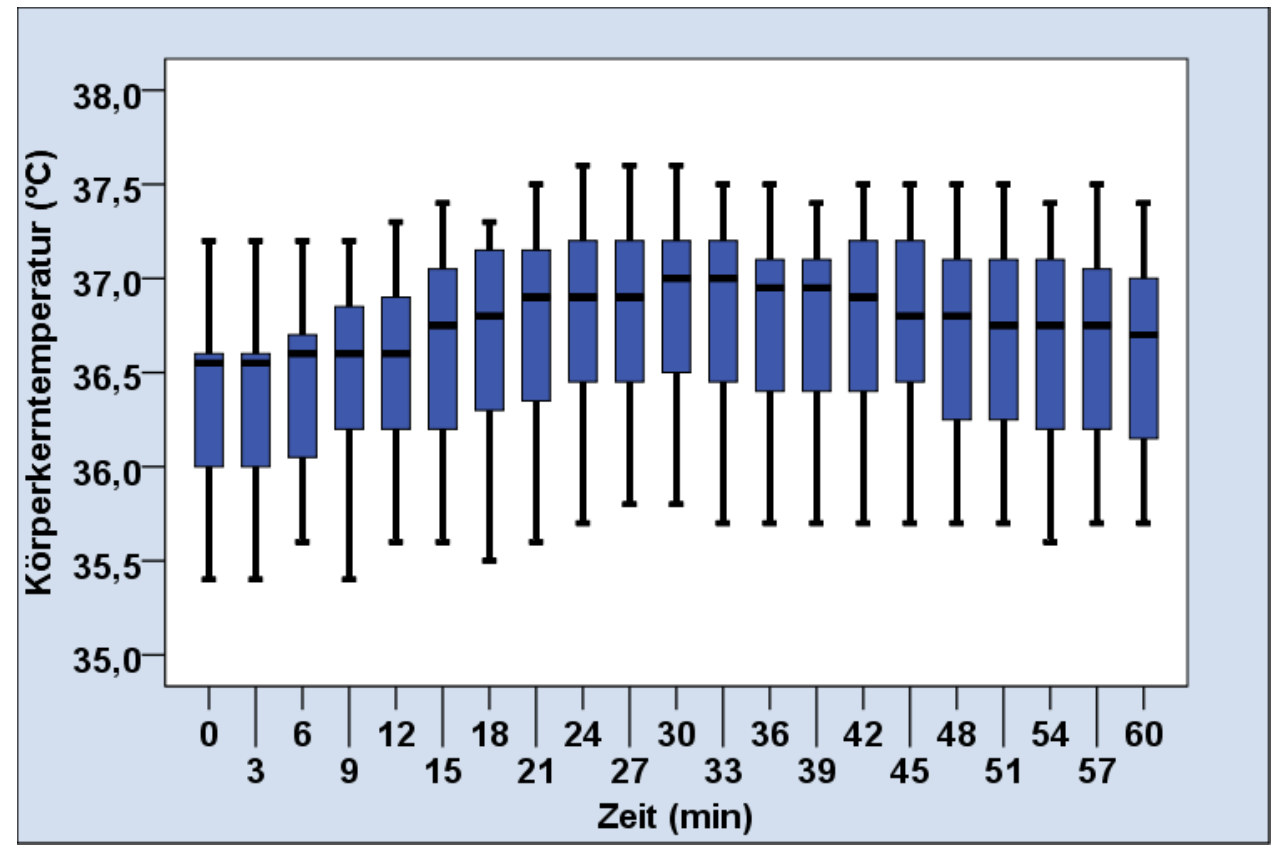

Abbildung 22: Körperkerntemperatur im Versuchteil mit Vliesdecke

Tabelle 13: Wilcoxon-Test Körperkerntemperatur Luftwärmer / Vliesdecke

\begin{tabular}{|l|r|r|r|r|r|}
\hline & $\begin{array}{c}\text { Körper KT T0 - } \\
\text { Körper KT T0 }\end{array}$ & $\begin{array}{c}\text { Körper KT T21 - } \\
\text { Körper KT T21 }\end{array}$ & $\begin{array}{c}\text { Körper KT T30 - } \\
\text { Körper KT T30 }\end{array}$ & $\begin{array}{c}\text { Körper KT T45 - } \\
\text { Körper KT T45 }\end{array}$ & $\begin{array}{c}\text { Körper KT T60 - } \\
\text { Körper KT T60 }\end{array}$ \\
\hline $\begin{array}{l}\text { Asymptotische Signifikanz } \\
\text { (2-seitig) }\end{array}$ & $\mathbf{7 3 6}$ & $\mathbf{9 5 9}$ & $\mathbf{9 1 8}$ & $\mathbf{7 0 3}$ & $\mathbf{8 1 1}$ \\
\hline
\end{tabular}




\subsubsection{Mittlere Hauttemperatur (MHT)}

Bei der Mittleren Hauttemperatur, die aus der Formel nach Ramanathan berechnet wurde, zeigten sich zu den Zeitpunkten 30, 45 und 60 Minuten signifikante Unterschiede zwischen beiden Versuchsteilen. In den Medianen waren die gemessenen und schließlich errechneten mittleren Hauttemperaturen im Versuchsteil A "Luftwärmer" signifikant höher, als im Versuchsteil B "Vliesdecke“, dieses ist in Tabelle 14 ersichtlich. In beiden Versuchsreihen stieg die mittlere Hauttemperatur, im Median, nach Beginn der Erwärmung. Der Anstieg der mittleren Hauttemperatur fiel jedoch im Versuchsteil „Luftwärmer“ höher aus. Hier zeigt sich, dass bereits zum Zeitpunkt 30 Minuten die mittlere Hauttemperatur der Probanden im Versuchsteil „Luftwärmer" um 1,9 ${ }^{\circ} \mathrm{C}$ höher war, als im Versuchsteil „Vliesdecke“. Zum Zeitpunkt 45 Minuten beträgt die Temperaturdifferenz der Mediane zwischen beiden Versuchsteilen weiter $1,9^{\circ} \mathrm{C}$. Nach 60 Minuten jedoch sinkt die mittlere Hauttemperatur im Versuchsteil „Vliesdecke“ wieder, während sie im Versuchsteil „Luftwärmer“ weiter konstant bleibt. Hieraus resultiert ein Temperaturunterschied von $+1{ }^{\circ} \mathrm{Celsius}$ im Vergleich $z u$ den Probanden, die mit der Vliesdecke gewärmt wurden.

Tabelle 14: Mediane Mittlere Hauttemperatur

\begin{tabular}{|l|c|c|}
\hline Zeitpunkt & $\begin{array}{l}\text { Mediane } \\
\text { Luftwärmer }\end{array}$ & Mediane Vliesdecke \\
\hline 21 Minuten & $27,6^{\circ} \mathrm{C}$ & $27,2^{\circ} \mathrm{C}$ \\
\hline 30 Minuten & $30,2^{\circ} \mathrm{C}$ & $28,3^{\circ} \mathrm{C}$ \\
\hline 45 Minuten & $31,1^{\circ} \mathrm{C}$ & $29,2^{\circ} \mathrm{C}$ \\
\hline 60 Minuten & $31,1^{\circ} \mathrm{C}$ & $28,9^{\circ} \mathrm{C}$ \\
\hline
\end{tabular}




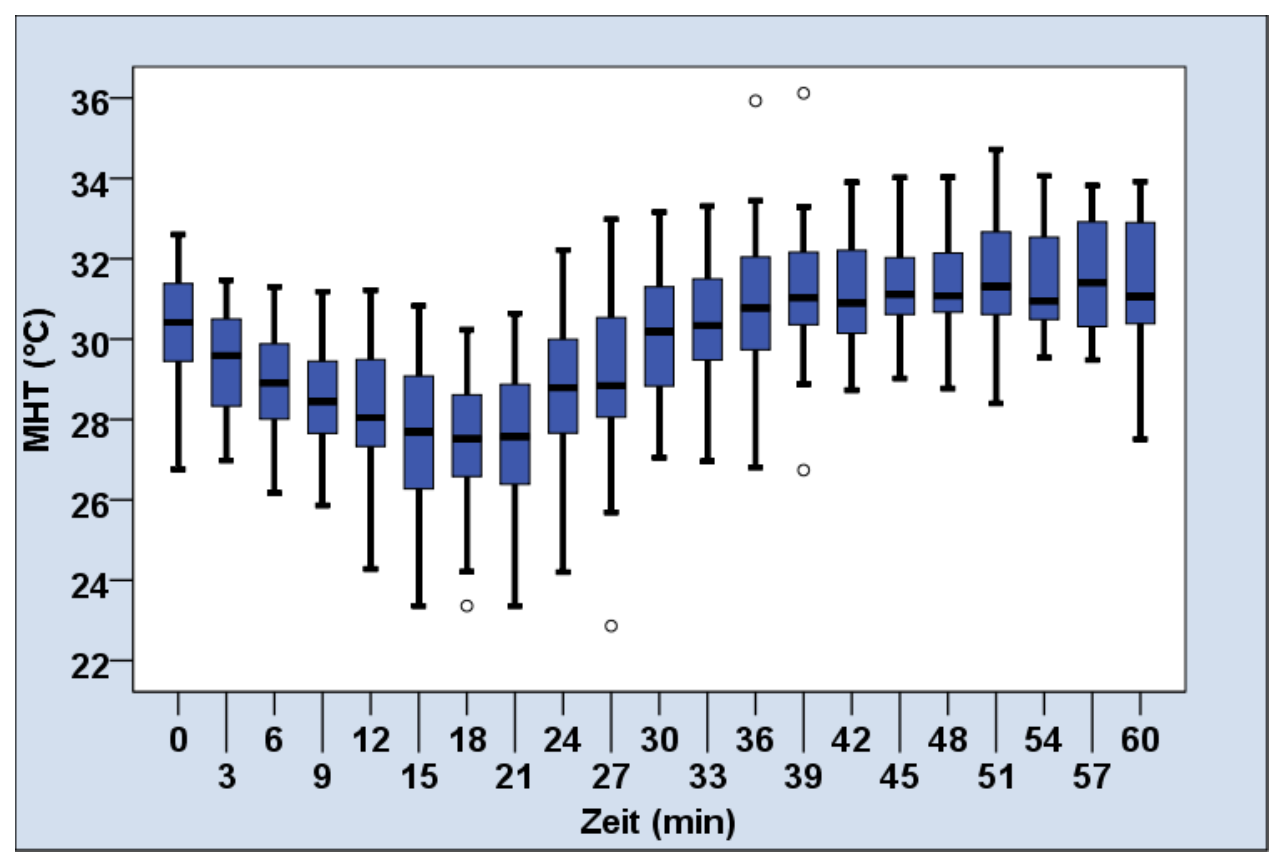

Abbildung 23: Mittlere Hauttemperatur im Versuchsteil mit konvektiver Luftwärmung

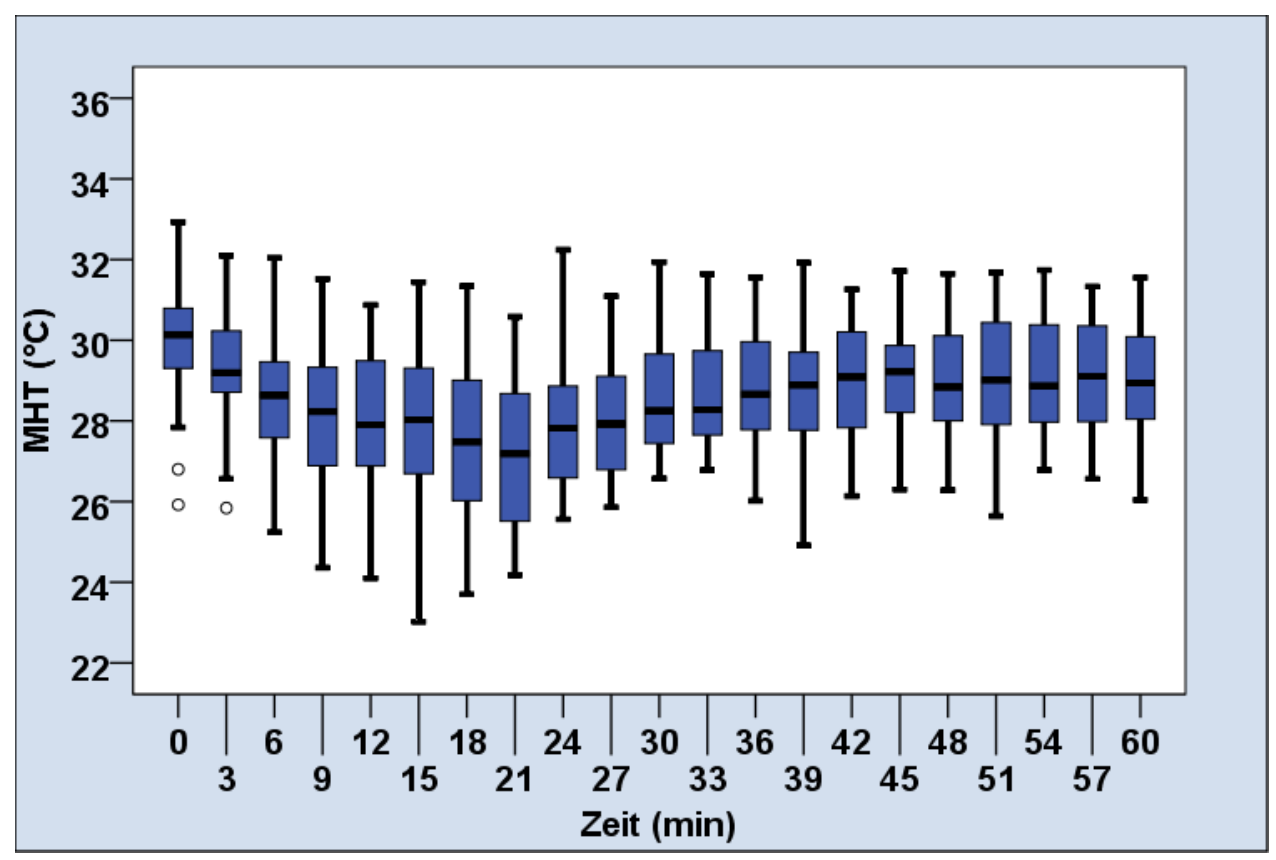

Abbildung 24: Mittlere Hauttemperatur im Versuchteil mit Vliesdecke

Tabelle 15: Wilcoxon-Test Mittlere Hauttemperatur Luftwärmer / Vliesdecke

\begin{tabular}{|l|r|r|r|r|r|}
\hline & $\begin{array}{c}\text { MHT T0 - MHT } \\
\text { T0 }\end{array}$ & $\begin{array}{r}\text { MHT T21 - MHT } \\
\text { T21 }\end{array}$ & $\begin{array}{r}\text { MHT T30 - MHT } \\
\text { T30 }\end{array}$ & $\begin{array}{c}\text { MHT T45 - MHT } \\
\text { T45 }\end{array}$ & $\begin{array}{c}\text { MHT T60 - MHT } \\
\text { T60 }\end{array}$ \\
\hline $\begin{array}{l}\text { Asymptotische Signifikanz } \\
\text { (2-seitig) }\end{array}$ & $\mathbf{6 4 0}$ &, $\mathbf{2 1 0}$ &, 000 &, 000 &, 000 \\
\hline
\end{tabular}




\subsubsection{Mittlere Körpertemperatur (MKT)}

Bei der Mittleren Körpertemperatur zeigten sich zu den Zeitpunkten 30, 45 und 60 Minuten signifikante Unterschiede. Auch hier waren die errechneten Temperaturwerte im Median im Versuchsteil A „Luftwärmer" höher als im Versuchsteil B „Vliesdecke“. Die Mediane der Körpertemperatur sind in der Tabelle 16 dargestellt. Es zeigt sich hier, dass die Mediane der mittleren Körpertemperatur bei dem Versuchsteil „Luftwärmer" bereits zum Zeitpunkt 30 Minuten um $0,7^{\circ} \mathrm{C}$ höher war als der Median im Versuchsteil „Vliesdecke“. Nach 45 Minuten betrug der Temperaturunterschied der Mediane dann bereits $0,9{ }^{\circ} \mathrm{C}$ und nach 60 Minuten war die mittlere Körpertemperatur dann bei dem Probandenkollektiv im Versuchsteil „Luftwärmer" um $1{ }^{\circ} \mathrm{C}$ im Median höher, als im Versuchsteil „Vliesdecke“.

Tabelle 16: Mediane Mittlere Körpertemperatur

\begin{tabular}{|l|c|c|}
\hline Zeitpunkt & $\begin{array}{l}\text { Mediane } \\
\text { Luftwärmer }\end{array}$ & Mediane Vliesdecke \\
\hline 21 Minuten & $33,4^{\circ} \mathrm{C}$ & $33,1^{\circ} \mathrm{C}$ \\
\hline 30 Minuten & $34,4^{\circ} \mathrm{C}$ & $33,7^{\circ} \mathrm{C}$ \\
\hline 45 Minuten & $34,8^{\circ} \mathrm{C}$ & $33,9^{\circ} \mathrm{C}$ \\
\hline 60 Minuten & $34,9^{\circ} \mathrm{C}$ & $33,9^{\circ} \mathrm{C}$ \\
\hline
\end{tabular}

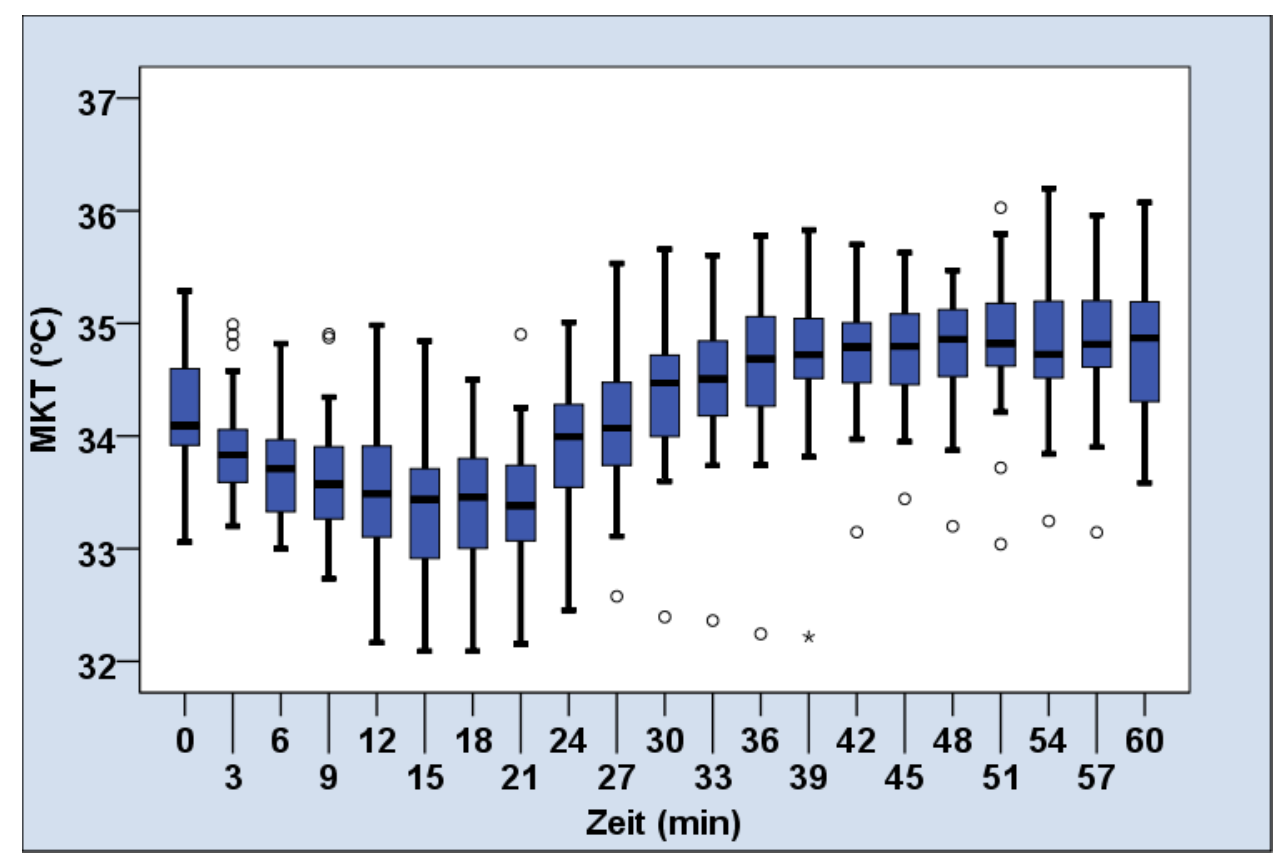

Abbildung 25: Mittlere Körpertemperatur im Versuchsteil mit konvektiver Luftwärmung 


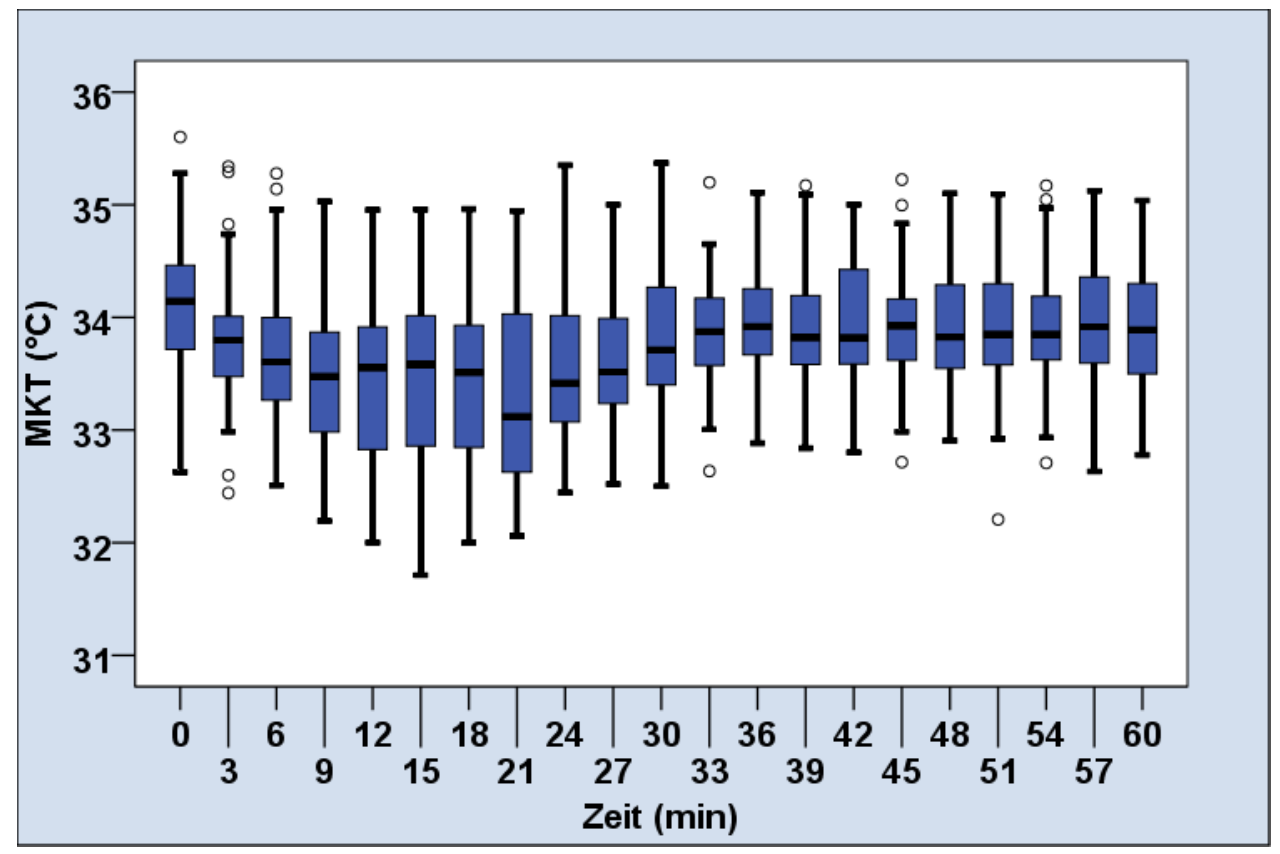

Abbildung 26: Mittlere Körpertemperatur im Versuchteil mit Vliesdecke

Tabelle 17: Wilcoxon-Test Mittlere Körpertemperatur Luftwärmer / Vliesdecke

\begin{tabular}{|l|r|r|r|r|r|}
\hline & $\begin{array}{c}\text { MKT T0 - MKT } \\
\text { T0 }\end{array}$ & $\begin{array}{r}\text { MKT T21 - MKT } \\
\text { T21 }\end{array}$ & $\begin{array}{r}\text { MKT T30 - MKT } \\
\text { T30 }\end{array}$ & $\begin{array}{c}\text { MKT T45 - MKT } \\
\text { T45 }\end{array}$ & $\begin{array}{c}\text { MKT T60 - MKT } \\
\text { T60 }\end{array}$ \\
\hline $\begin{array}{l}\text { Asymptotische Signifikanz } \\
\text { (2-seitig) }\end{array}$ & $\mathbf{8 3 7}$ &, 722 &, 001 &, 000 &, 000 \\
\hline
\end{tabular}

\subsubsection{Gesamtkörperwärmemenge (GKW)}

Die Auswertung der Mediane der Gesamtkörperwärmemenge zeigte zunächst in beiden Versuchsteilen einen Abfall bis zum Zeitpunkt 21 Minuten. Im Verlauf des Versuchsteils A „Luftwärmer“ kam es dann zu einer Zunahme der Gesamtkörperwärmemenge, während die Gesamtkörperwärmemenge im Versuchsteil B „Vliesdecke“ auf einem Niveau stabil blieb.

Signifikanzen zeigten sich zu den Zeitpunkten 30, 45 und 60 Minuten. Hier waren die Mediane der Werte im Versuchsteil A "Luftwärmer" höher, als im Versuchsteil B „Vliesdecke“. 
Tabelle 18: Mediane Gesamtkörperwärmemenge

\begin{tabular}{|l|c|c|}
\hline Zeitpunkt & \multicolumn{1}{|l|}{$\begin{array}{l}\text { Mediane } \\
\text { Luftwärmer }\end{array}$} & $9107,3 \mathrm{~kJ}$ \\
\hline 21 Minuten & $9111,2 \mathrm{~kJ}$ & $9299,6 \mathrm{~kJ}$ \\
\hline 30 Minuten & $9506,9 \mathrm{~kJ}$ & $9301,1 \mathrm{~kJ}$ \\
\hline 45 Minuten & $9550,8 \mathrm{~kJ}$ & $9288,2 \mathrm{~kJ}$ \\
\hline 60 Minuten & $9599,1 \mathrm{~kJ}$ & Vliesdecke \\
\hline
\end{tabular}

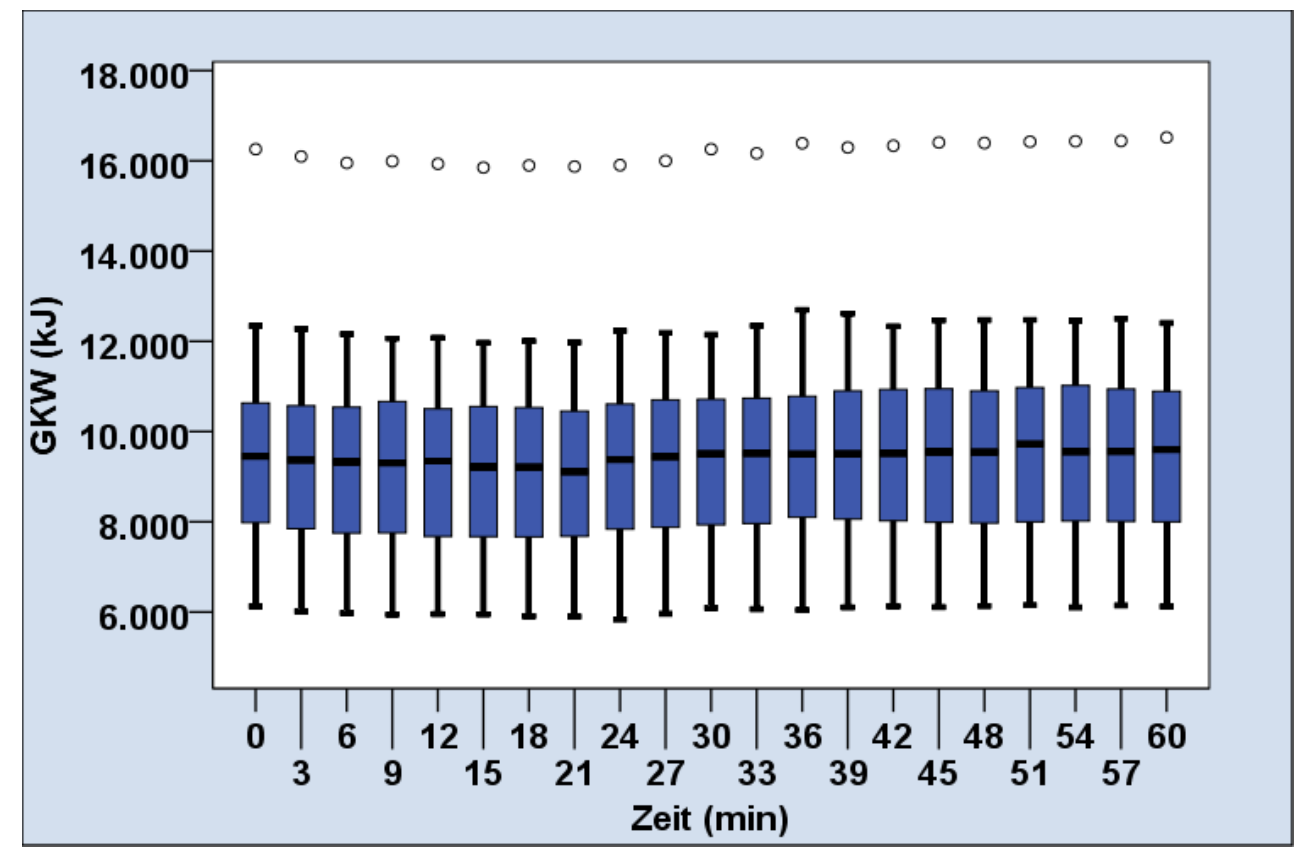

Abbildung 27: Gesamtkörperwärmemenge im Versuchsteil mit konvektiver Luftwärmung 


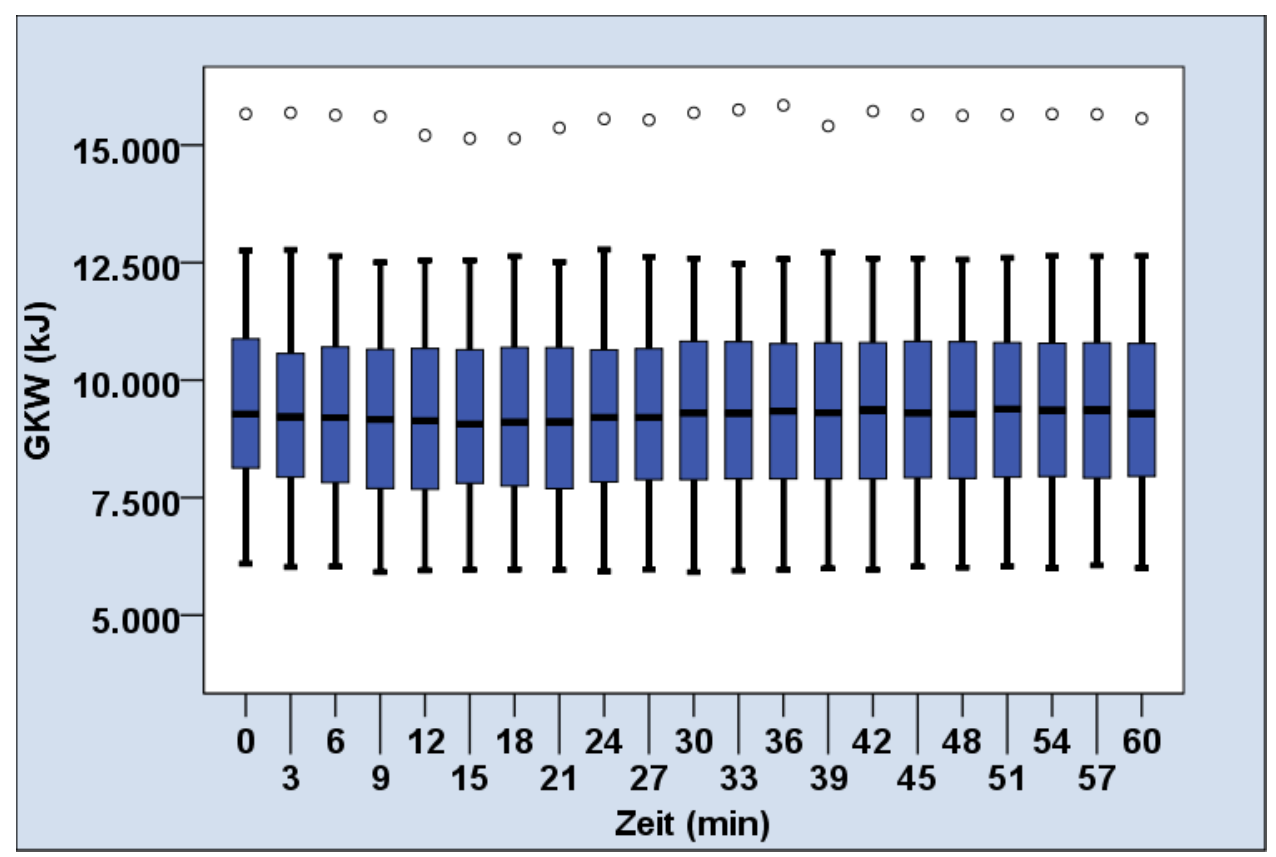

Abbildung 28: Gesamtkörperwärmemenge im Versuchsteil mit Vliesdecke

Tabelle 19: Wilcoxon-Test Gesamtkörperwärmemenge Luftwärmer / Vliesdecke

\begin{tabular}{|l|r|r|r|r|r|}
\hline & $\begin{array}{c}\text { GKW T0 - GKW } \\
\text { T0 }\end{array}$ & $\begin{array}{c}\text { GKW T21 - GKW } \\
\text { T21 }\end{array}$ & $\begin{array}{r}\text { GKW T30 - GKW } \\
\text { T30 }\end{array}$ & $\begin{array}{c}\text { GKW T45 - GKW } \\
\text { T45 }\end{array}$ & $\begin{array}{c}\text { GKW T60 - GKW } \\
\text { T60 }\end{array}$ \\
\hline $\begin{array}{l}\text { Asymptotische Signifikanz } \\
\text { (2-seitig) }\end{array}$ & ,926 &, 940 &, 001 &, 000 &, 000 \\
\hline
\end{tabular}

\subsubsection{Numerische Ratingskala (NRS)}

In der Auswertung des subjektiven Empfindens mittels der Numerischen Ratingskala zeigten sich nach Beginn des Wärmeverfahrens im Versuchsteil A „Luftwärmer" ebenso deutlich geringere NRS-Werte, als im Versuchsteil B „Vliesdecke“. Im Median wurde zum Zeitpunkt 21 Minuten in beiden Versuchsteilen der NRS-Wert mit 5 angegeben. Unter der Wärmung mittels konvektivem Luftwärmer wurden im Verlauf das Kältegefühl zu den Zeitpunkten 30, 45 und 60 Minuten im Median mit 2 auf der Numerischen Ratingskala angegeben. Im Vergleich hierzu betrug der NRS-Median bei der Wärmung mittels Vliesdecke zu den gleichen Zeitpunkten 4. Somit waren die Unterschiede zu den Zeitpunkten 30, 45 und 60 Minuten hoch signifikant. 


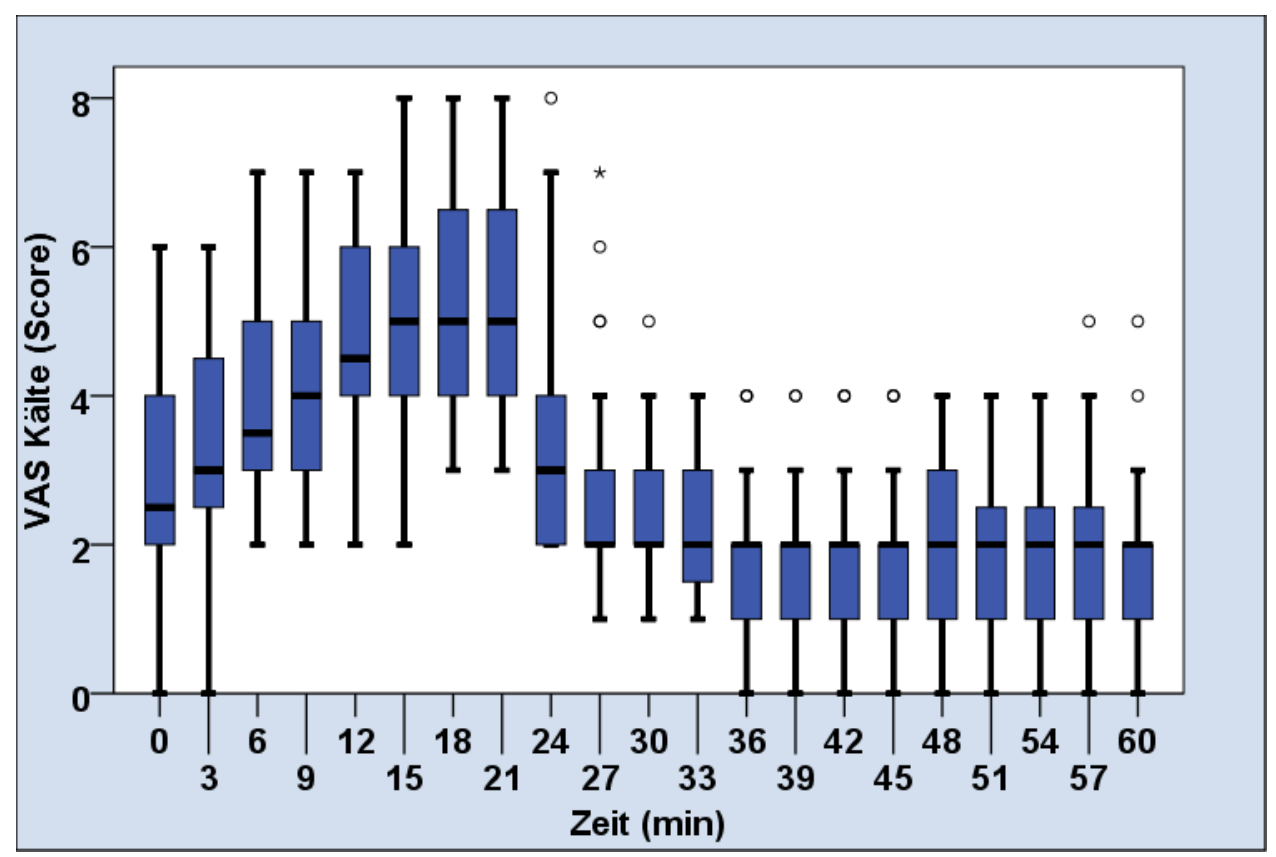

Abbildung 29: Numerische Ratingskala im Versuchsteil mit konvektiver Luftwärmung

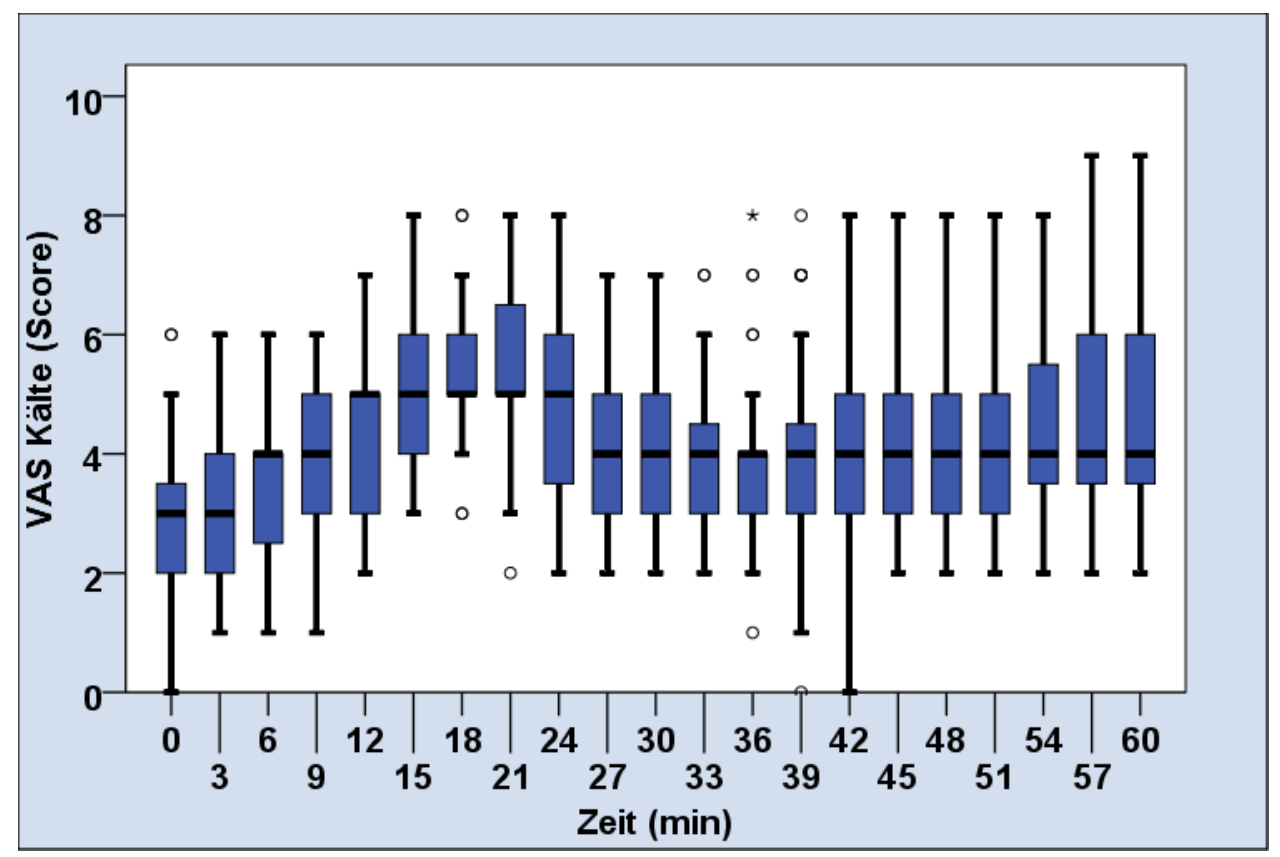

Abbildung 30: Numerische Ratingskala im Versuchteil mit Vliesdecke

Tabelle 20: Wilcoxon-Test Numerische Ratingskala

\begin{tabular}{|l|r|r|r|r|r|}
\hline & $\begin{array}{l}\text { NRS Kälte T0 - } \\
\text { NRS Kälte T0 }\end{array}$ & $\begin{array}{c}\text { NRS Kälte T21 - } \\
\text { NRS Kälte T21 }\end{array}$ & $\begin{array}{c}\text { NRS Kälte T30 - } \\
\text { NRS Kälte T30 }\end{array}$ & $\begin{array}{c}\text { NRS Kälte T45 - } \\
\text { NRS Kälte T45 }\end{array}$ & $\begin{array}{c}\text { NRS Kälte T60 - } \\
\text { NRS Kälte T60 }\end{array}$ \\
\hline $\begin{array}{l}\text { Asymptotische Signifikanz (2- } \\
\text { seitig) }\end{array}$ & $\mathbf{4 9 2}$ &, 916 &, 000 &, 000 &, 000 \\
\hline
\end{tabular}




\subsubsection{Subjektive Bewertung}

Die Auswertung der subjektiven Bewertung der Effektivität der Wärmeverfahren ist den Tabellen 21 und 22 zu entnehmen. Es zeigt sich, dass 90,62\% der Probanden die Wärmung mittels Luftwärmer als „deutlich“ beurteilten, während bei der Wärmung mit der Vliesdecke nur $15,6 \%$ diese Einschätzung vertraten. $50 \%$ gaben an, dass die Vliesdecke nur gering wärmen würde. Diese Bewertung wurde hinsichtlich des konvektiven Luftwärmers von keinem Probanden gemacht.

Tabelle 21: Subjektive Bewertung im Versuchsteil mit konvektiver Luftwärmung

\begin{tabular}{|l|r|r|}
\hline & Häufigkeit & Prozent \\
\hline hat mäßig gewärmt & 3 & 9,38 \\
hat deutlich gewärmt & 29 & 90,62 \\
Gesamtsumme & 32 & 100,00 \\
\hline
\end{tabular}

Tabelle 22: Subjektive Bewertung Vliesdecke

\begin{tabular}{|l|r|r|}
\hline & Häufigkeit & Prozent \\
\hline hat nur gering gewärmt & 16 & 50,0 \\
hat mäßig gewärmt & 11 & 34,4 \\
hat deutlich gewärmt & 5 & 15,6 \\
Gesamtsumme & 32 & 100,0 \\
\hline
\end{tabular}

\subsubsection{Bedsite-Shivering-Assessment-Scale (BSA)}

Es konnte nur bei vier Probanden ein Kältezittern beobachtet und somit im Versuchsablauf eine Zuordnung nach der o.g. Skala vorgenommen werden. Der Median betrug daher über alle Probanden in allen Versuchsteilen 0.

Die Auswertung hinsichtlich signifikanter Unterschiede des Bedsite-Shivering-AssessmentScale war damit nicht sinnvoll und wurde nicht durchgeführt.

Bis zum Zeitpunkt 21 Minuten ließen sich dabei in beiden Versuchsteilen jeweils zwei Versuchsteilnehmer beobachten, bei denen der BSA mit zwei bewertet wurde. Im Verlauf der jeweiligen Wärmemethoden ließ sich im Versuchsteil konvektiver Luftwärmer ab dem Zeitunkt 30 Minuten kein Shivering mehr feststellen. Im Versuchsteil Vliesdecke konnten bis zum Zeitpunkt T60 durchgehend zwei Probanden mit BSA-Werten zwischen 1 und 2 beschrieben werde 


\section{Diskussion}

\subsection{Diskussion der Methodik}

\subsubsection{Diskussion der Wärmemethoden}

Mit dieser Studie wurden zwei unterschiedliche Wärmemethoden überprüft. Ein passives, isolierendes Verfahren, die Vlies-Einmaldecke, und ein aktives, konvektives Verfahren, wie es in der innerklinischen Patientenversorgung zum Einsatz kommt.

In der präklinischen Notfallmedizin werden bei der Vorsorge und Behandlung von hypothermen Zuständen aktuell überwiegend passive Wärmemethoden, wie Rettungsdecken, Wolldecken und Vlies-Einmaldecken, verwendet. So konnten Heyne et.al. in einer unveröffentlichten Studie nachweisen, dass 85\% der an der Patientenversorgung teilnehmenden Feuerwehren in Deutschland passive Wärmemethoden einsetzen. Die in dieser Probandensimulation ausgewählte Vlies-Einmaldecke ist aktuell am Markt erhältlich und wird von dem Hersteller als Einmalartikel und insbesondere für den präklinischen Einsatz hergestellt (Reintex 2013). Dieses Verfahren kann somit als aktueller Standard und etabliertes Verfahren in der präklinischen Behandlung von verletzten oder erkrankten Personen in deutschen Rettungsdiensten angesehen werden. Diese Produkte gelten als sicher in der Anwendung und kommen ubiquitär zum Einsatz. Der Nachweis zur Wirksamkeit unter präklinischen Bedingungen wurde jedoch vor dieser Studie nur in einer Studie mit einem sehr kleinen Probandenkollektiv $(n=5)$ erbracht, jedoch nicht mit anderen aktiven Verfahren verglichen (Thomassen et al. 2011).

Als zweites Wärmeverfahren kam ein konvektiver Luftwärmer zur Anwendung, wie er standardisiert und weltweit innerklinisch zur Therapie und Prophylaxe von hypothermen Zuständen eingesetzt wird. Durch zahlreiche Untersuchungen konnte gezeigt werden, dass dieses Verfahren unter innerklinischen Bedingungen sicher und hoch effektiv ist und darüberhinaus allen rein passiven Wärmemethoden deutlich überlegen ist (Scherer 1997, Heuer 2000, Bräuer et al. 2002).

Bisher existiert jedoch nur eine Studie, die die Wirksamkeit eines konvektiven Luftwärmer unter präklinischen Bedingungen überprüft hat. Es handelte sich um ein Gerät zum Beheizen 
von Zelten o.ä., welches durch seine Bauart für den präklinischen Einsatz, jedoch nicht primär für die Verwendung am Patienten vorgesehen ist. In der Studie konnte gezeigt werden, dass eine Erwärmung hiermit unter präklinischen Bedingungen möglich ist. Das verwendete Gerät wurde von den Autoren der zitierten Studie jedoch als nicht sicher für die Verwendung an Patienten beurteilt, da die Temperaturen im Luftstrom des Gerätes deutlich höher als $43^{\circ} \mathrm{C}$ gemessen wurden und somit nach Einschätzung des Autors mögliche Risiken für thermische Schäden der Haut bestanden (Jebens 2014).

Somit wurden in der aktuellen Studie zwei etablierte und in innerklinischen Untersuchungen als sicher bewertete Verfahren überprüft und hinsichtlich der Unterschiede in der Wirksamkeit bewertet.

\subsubsection{Diskussion des Studiendesigns}

Die Durchführung von randomisierten Studien in der präklinischen Notfallmedizin ist schwierig und wird oft durch die fehlende Einwilligungsfähigkeit der Patienten und die hohe Dynamik und Varianz bei Notfallsituationen begrenzt. Daher wird häufig auf retrospektive Studien ausgewichen (Kreimeier et al.). Eine Alternative können Simulationen unter standardisierten Bedingungen bilden, wie in dem vorliegenden Fall.

Bei der hier beschriebenen Studie handelt es sich um eine randomisierte, experimentelle, prospektive Studie mit gesunden Probanden. Bei der Definition eines Studiendesigns für eine derartige Studie ist eine möglichst hohe Standardisierung erforderlich, um bei relativ niedrigen Probandenkollektiven verwertbare Aussagen zu treffen. Gelingt dieses nicht, ist die Fallzahl der durchgeführten Untersuchung deutlich zu erhöhen.

In der vorliegenden Studie waren alle Abschnitte der Versuche standardisiert und wurden streng eingehalten. Die Umgebungstemperatur und die zeitliche Abfolge der Versuche folgten ohne Abweichungen dem Studienprotokoll.

\subsubsection{Limitationen des Studiendesigns}

Natürlich unterscheiden sich diese Laborbedingungen von den realen Gegebenheiten an einer Unfallstelle. Umweltbedingungen wie unterschiedliche Windstärken, Differenzen der 
Luftfeuchtigkeit und Wetterverhältnisse, wie Regen oder Schnee, wurden hier nicht abgebildet.

Gleiches gilt für unterschiedliche Temperaturen. Doch die gewählte Umgebungstemperatur von $3{ }^{\circ} \mathrm{C} \pm 1{ }^{\circ} \mathrm{C}$ wurde, im Vergleich zu einer durchschnittlichen Temperatur in Deutschland von $9,2^{\circ} \mathrm{C}$, deutlich niedriger gewählt (Luftemperatur 2015). Somit sind die erhobenen Ergebnisse insbesondere in Hinblick auf die Wirksamkeit bei den präklinisch herrschenden Temperaturen eindrücklich und dürften an realen Einsatzstellen, bei den dort überwiegend herrschenden höheren Temperaturen, zumindest entsprechend ausfallen.

Die mit Unterwäsche, Shorts und T-Shirt gewählte Kleidung der Probanden wird nicht immer der in Mitteleuropa bei Verkehrsunfällen anzutreffenden Bekleidung entsprechen. Diese wurde jedoch bewusst so gewählt, um bei den Probanden einen möglichst hohen Wärmeverlust zu erzielen. Zudem kann es auch im Rahmen von Verkehrsunfällen zu einer Zerstörung der Bekleidung kommen, bzw. die Kleidung wird, spätestens für die medizinische Versorgung vom Rettungsdienstpersonal entfernt. Pathophysiologische Folgen auf die Körpertemperatur durch schwere Verletzungen, hypovolämische Schockzustände, SchädelHirn-Traumata und die für die medizinische Versorgung erforderlichen invasiven Maßnahmen, konnten bei einem gesunden Probandenkollektiv nicht simuliert werden.

Ein Abfall der Körpertemperatur, insbesondere der Körperkerntemperatur, ist jedoch in einem derartigen realen Patientenkollektiv deutlich höher zu erwarten (Mommsen et al. 2013). Die gewählte Kleidung verfälscht somit die Ergebnisse nicht und ist lediglich der Simulation an gesunden Probanden geschuldet.

\subsubsection{Diskussion Probandenkollektiv}

Betrachtet man die Ergebnisse der Gesundheitsberichterstattung des Bundes in Bezug auf das Unfallgeschehen bei Erwachsenen in der Bundesrepublik Deutschland, so fällt unter anderem auf, dass zwei Drittel der beteiligten Personen an schweren Verkehrsunfällen Männer sind (Unfallbericht 2013).

Auch in der Auswahl der teilnehmenden Probanden findet sich diese Geschlechterverteilung näherungsweise wieder. So sind auch in der durchgeführten Studie zur Untersuchung der Wirksamkeit von unterschiedlichen Wärmemethoden unter präklinischen Bedingungen die 
männlichen Probanden mit 56,3\% in der Mehrheit und bilden somit nahezu zwei Drittel der teilnehmenden Testpersonen. 43,8 \% der Probanden sind Frauen, dieses entspricht folglich auch näherungsweise dem Anteil von Frauen in der beschriebenen Statistik der Gesundheitsberichterstattung.
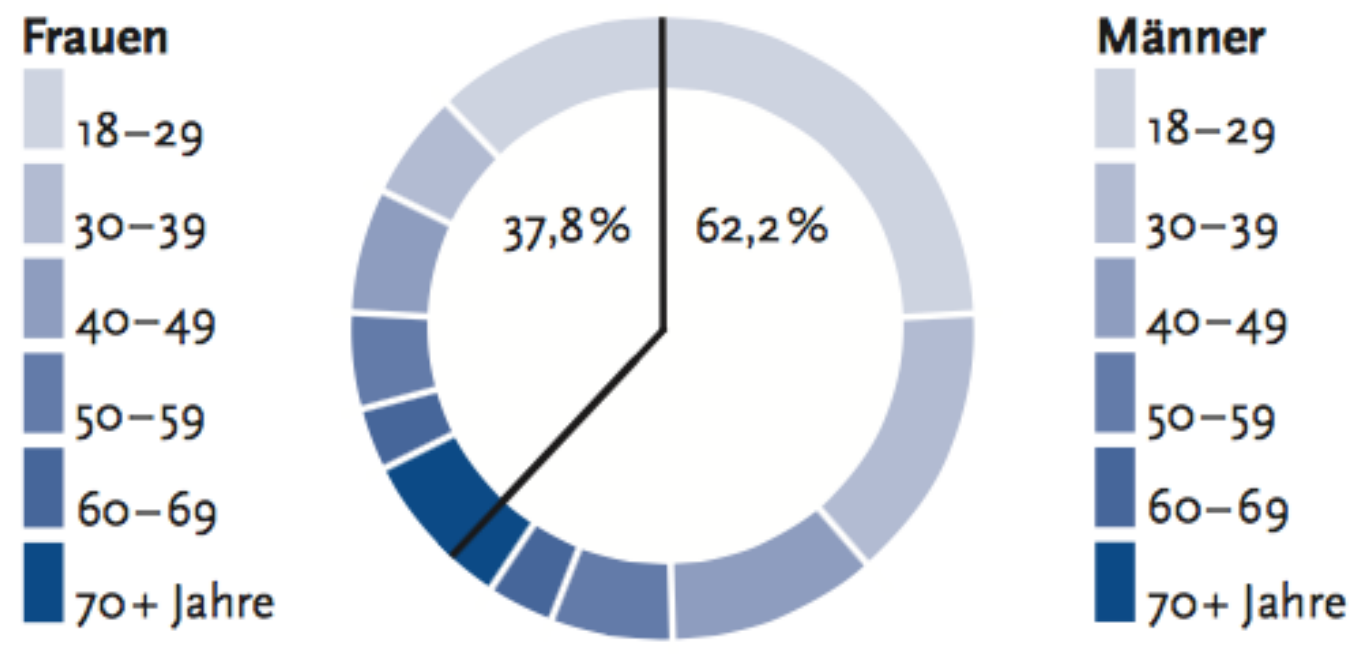

Abbildung 31: Unfallgeschehen nach Alter und Geschlecht in Deutschland. (Unfallbericht 2013)

Bei der Altersverteilung in der Statistik der Bundesrepublik bilden die 18-39-Jährigen mit ca. 55\% den Hauptteil der verletzten Personen (Unfallbericht 2013). Dieses findet sich im Probandenkollektiv nicht wieder. Hier wird der größte Anteil durch das Alterskollektiv der 40-49-jährigen Probanden gebildet. Auch finden sich keine Personen über 60 Jahre in diesem Probandenkollektiv, was eine Folge der Ausschlusskriterien ist.

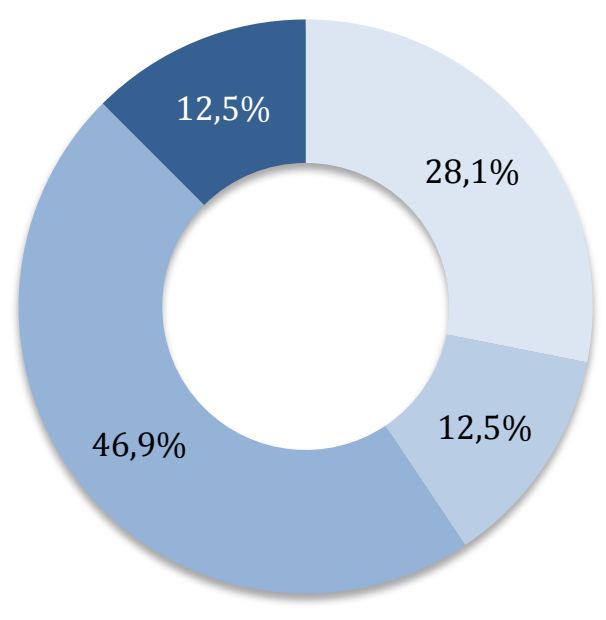

18-29 Jahre

30-39 Jahre

40-49 Jahre

50-59 Jahre 
In der Gesamtbetrachtung bleibt jedoch festzustellen, dass Probanden aus allen Altersstufen, die gemäß den Ein- und Ausschlusskriterien an der Studie teilnehmen durften, an der Studie teilgenommen haben.

\subsubsection{Diskussion Probandensicherheit}

Die durchgeführten Untersuchungen erbrachten keinen Hinweis darauf, dass die teilnehmenden Probanden durch die verwendeten Wärmemethoden irgendwelchen gesundheitlichen Gefahren ausgesetzt waren. Die gemessenen Temperaturen an der Hautoberfläche, insbesondere bei der Verwendung des konvektiven Luftwärmer, waren durchgehend kleiner als $43{ }^{\circ} \mathrm{C}$. Das Risiko von thermischen Schäden der Haut war zu keiner Zeit gegeben. Beide Verfahren können somit für den Patienten sicher unter präklinischen Bedingungen zur Anwendung kommen.

\subsection{Diskussion der Ergebnisse}

\subsubsection{Vitalparameter}

Bei der statistischen Auswertung der gemessenen Vitalparameter zeigten sich signifikante Abweichungen zwischen den beiden Versuchsreihen. So zeigte sich zum Testzeitpunkt 30 min die Herzfrequenz der Probanden, die mit dem konvektiven Luftwärmer gewärmt wurden, signifikant niedriger (Median 68,5/min zu 76,5/min). Im weiteren Verlauf des Versuches traten bei diesem Parameter keine Unterschiede mehr auf.

Ähnliche Verläufe zeigten sich auch bei den anderen gemessenen Vitalparametern. So konnten auch bei den gemessenen systolischen, diastolischen und Mitteldruckwerten sowie bei den Werten der peripheren Sauerstoffsättigung zu unterschiedlichen Zeiten grenzwertig signifikante bis signifikante Unterschiede gemessen werden.

So ist im Versuchsteil „Luftwärmer" der systolische Blutdruck zu den Zeitpunkten 30 min und $60 \mathrm{~min}$ grenzwertig signifikant niedriger (Median $128 \mathrm{mmHg}$ zu 131,5 mmHg und 130,5 $\mathrm{mmHg}$ zu 133,5 mmHg).

Ähnliche Ergebnisse konnten auch in den Arbeiten von Jebens und Hynson et.al. gefunden werden (Hynson et al. 1993, Jebens 2014). In gleicher Weise zeigte sich bei den aktiv gewärmten Patienten eine Erniedrigung des Blutdrucks. So waren in der Untersuchung von 
Jebens die systolischen Blutdrücke bei den Probanden, die mit einem konvektiven Luftwärmer gewärmt wurden, zu den Zeitpunkten $30 \mathrm{~min}, 45 \mathrm{~min}$ und $60 \mathrm{~min}$ signifikant niedriger.

Bei der Überprüfung des diastolischen Blutdrucks konnten zum Zeitpunkt 60min signifikant niedrigere Blutdrücke (Median $81,55 \mathrm{mmHg}$ zu $87 \mathrm{mmHg}$ ) im Warmluftversuch nachgewiesen werden.

Ähnliche Werte finden sich auch bei der statistischen Auswertung der gemessenen Mitteldruckwerte. Zum Zeitpunkt 21 min findet sich bei den aktiv gewärmten Probanden grenzwertig signifikant niedrigere Mitteldruckwerte (Median 94,5 mmHg zu 98,0 mmHg) und zum Zeitpunkt 60 min signifikant niedrigere Mitteldruckwerte (Median $96 \mathrm{mmHg} z u$ $100 \mathrm{mmHg}$ ).

Eine mögliche Erklärung für diese Unterschiede bei den Blutdrücken der Versuchsreihen ist, dass durch die aktive Wärmung eine begonnene Zentralisierung mit einer Engstellung der peripheren Gefäße abgeschwächt oder aufgehoben wird und der hierdurch bedingte Abfall des peripheren Widerstandes eine konsekutive Erniedrigung der Blutdruckwerte zur Folge hat. Zudem führt gegenregulatorisches Kältezittern als Folge des Kältestresses zu einem Anstieg des mittleren arteriellen Mitteldrucks (Giesbrecht 2000, Zink et al. 2004).

Somit geben die gemessenen Blutdruckwerte zumindest Hinweise darauf, dass die Wärmung mittels konvektivem Luftwärmer effektiver in der Behandlung des hypothermen Zustands ist.

Auch bei der peripher gemessenen Sauerstoffsättigung(SpO2) konnten zum Zeitpunkt 60 min signifikante Unterschiede nachgewiesen werden (Median 98,0\% zu 98,5\%). Korrelate in der Literatur ließen sich hierzu jedoch nicht finden. Mögliche Gründe hierfür könnten in einer veränderten Hautperfusion liegen. Betrachtet man die Medianwerte, so lässt sich aufgrund der geringen Unterschiede eine klinische Relevanz aber auch verneinen.

\subsubsection{Subjektive Beurteilung}

In der subjektiven Beurteilung mittels nummerischer Ratingskala und subjektiver Beurteilung der Effektivität der Wärmeverfahren zeigten sich signifikante Unterschiede.

Zwar gaben die Probanden nach Beginn beider Wärmeverfahren jeweils niedrigere Kälteempfindungen auf der nummerischen Ratingskala an, jedoch waren diese bereits zum Zeitpunkt 30 min, beim Luftwärmer-Verfahren, im Median um zwei Punkte niedriger. Aber 
auch für den Rest der statistischen Auswertung der Versuchsreihe bleibt dieser Unterschied im Median bei zwei Punkten. Somit ist auch in der subjektiven Bewertung der konvektive Luftwärmer der passiven Wärmemethode mittels Vlies-Einmaldecke überlegen. Im Literaturvergleich lassen sich diese Werte ebenso wiederfinden. So konnte auch Jebens in seiner Dissertationsarbeit zeigen, dass die aktive Wärmemethode mittels konvektivem Luftwärmer zu einem signifikanten Rückgang der Kältebewertung mittels nummerischer Ratingskala führte. Thomassen et al. konnten zeigen, dass es nach Beginn der Isolation mit einer Decke in einer kalten Umgebung zu einer Verbesserung der subjektiven Einschätzung des Kältegefühls kommt (Thomassen et al. 2011).

In der subjektiven Einschätzung der Effektivität beider Wärmeverfahren zeigte sich der konvektive Luftwärmer der Vlies-Einmaldecke deutlich überlegen. So meinten 90,62\%, dass der konvektive Luftwärmer "deutlich gewärmt" und 9,38\%, dass er zumindest „mäßig gewärmt" habe. Dagegen schätzten nur 15,6\% der Probanden die Wirkung der VliesEinmaldecke mit „,hat deutlich gewärmt" und 34,4\% mit „hat mäßig gewärmt" ein, während $50 \%$ die Wirkung nur mit „hat nur gering gewärmt“ beschrieben. Auch dieses entspricht im Literaturvergleich den zu erwartenden Ergebnissen und ist analog zu der Untersuchung von Jebens (Jebens 2014).

\subsubsection{Körperkerntemperatur}

Bei den gemessenen Körperkerntemperaturen zeigten sich in der statistischen Auswertung keine signifikanten Unterschiede zwischen den beiden Versuchsreihen. Eine signifikante Reaktion der Körperkerntemperatur konnte jedoch auch nicht erwartet werden, da diese Studie an gesunden Probanden durchgeführt wurde. Für den gesunden Menschen ist es essentiell, die Körperkerntemperatur auch innerhalb einer kalten Umgebung über einen langen Zeitraum konstant zu halten. Hierzu verfügt der Mensch über regulatorische Vorgänge wie Muskelzittern und die Vasokonstriktion (Hervey 1988, Sessler 1994).

Gleiche Ergebnisse konnte auch in der Simulation an einem gesunden Kollektiv von Jebens erhoben werden, so ließen sich keine Unterschiede bei der Auswertung der Körperkerntemperatur nachweisen (Jebens 2014). Thomassen et al. konnten in ihrer Studie an gesunden Probanden zwar signifikante Unterschiede in der Körperkerntemperatur nachweisen, jedoch war das Probandenkollektiv mit $n=8$ sehr klein (Thomassen et al. 2011). 
Die getesteten Wärmemethoden sollen u.a. bei schwer traumatisierten Unfallopfern Anwendung finden. Insbesondere nach schweren Verletzungen und durch erforderliche medizinische Maßnahmen kommt es zu Veränderungen in der Thermoregulation. Diese treten bei gesunden Probanden nicht oder nur nach einer sehr langen Kälteexposition auf (Mommsen et al. 2013).

\subsubsection{Mittlere Hauttemperatur}

Um die Temperaturveränderungen darzustellen, wurde daher u.a. die Mittlere Hauttemperatur nach der Formel von Ramanathan verwendet und statistisch ausgewertet. Hier zeigten sich nach Beginn der Wärmemethoden zum Zeitpunkt $30 \mathrm{~min}, 45 \mathrm{~min}$ und $60 \mathrm{~min}$ signifikante Unterschiede.

So war die Mittlere Hauttemperatur der Probanden, die mit dem konvektiven Luftwärmer gewärmt wurden, im Median um 2,2 ${ }^{\circ} \mathrm{C}$ zum Zeitpunkt $30 \mathrm{~min}$, bzw. um $1,9{ }^{\circ} \mathrm{C}$ und $2,2{ }^{\circ} \mathrm{C}$ zu den Zeitpunkten 45 min und 60 min höher.

$\mathrm{Zu}$ erkennen ist in den Boxplot-Kurven jedoch auch, dass die Vlies-Einmaldecke trotzdem eine Wirkung hat. So kommt es nach Beginn der Erwärmung mit der Decke zu keinem weiteren Abfall der mittleren Hauttemperatur im Median und im Verlauf schließlich wieder zu einem Anstieg. Auch in der Arbeit von Thomassen et al. wird dieses beschrieben. In einer innerklinischen Arbeit von Sessler et al. die an fünf gesunden Probanden in einer Umgebungstemperatur von $20,6{ }^{\circ} \mathrm{C}$ durchgeführt wurde, zeigten sich ebenfalls ähnliche Effekte (Sessler und Moayeri 1990). Ob diese Ergebnisse auf Traumapatienten zu übertragen sind, ist jedoch fraglich, da die Temperaturverluste durch die beschriebenen pathophysiologischen Vorgänge deutlich stärker ausfallen (Tsuei und Kearney 2004).

Diskutierbar ist die Frage, ob die Mittlere Hauttemperatur wie bei Ramanathan beschrieben durch die Verwendung von nur vier Messpunkten sicher ermittelt werden kann. Jirak et.al. beschreiben die Bestimmung der Mittleren Hauttemperatur mit dieser Formel für orientierende Untersuchungen jedoch als ausreichend (Jirak et al. 1975).

\subsubsection{Mittlere Körpertemperatur}

Aus der Körperkerntemperatur und der Mittleren Hauttemperatur wurde mit Hilfe der beschriebenen Formel die Mittlere Körpertemperatur errechnet und statistisch ausgewertet. 
Hier zeigen sich zu den Zeitpunkten $30 \mathrm{~min}, 45 \mathrm{~min}$ und $60 \mathrm{~min}$ signifikant höhere Mittlere Körpertemperaturen in der Versuchsreihe "konvektiver Luftwärmer". So ist die Temperatur bei den mit dem konvektiven Luftwärmer gewärmten Probanden zum Zeitpunkt 30 min um $0,7{ }^{\circ} \mathrm{C}$, zum Zeitpunkt $45 \mathrm{~min}$ um $0,9^{\circ} \mathrm{C}$ und zum Zeitpunkt 60 min um $1{ }^{\circ} \mathrm{C}$ höher. Auch in der Arbeit von Jebens findet sich eine signifikant höhere Mittlere Körpertemperatur in der Gruppe der Probanden, die mit dem konvektiven Luftwärmer gewärmt wurden.

\subsubsection{Gesamtkörperwärmemenge}

Schließlich wurde aus den Werten der Mittleren Körpertemperatur, dem Gewicht des Probanden und der spezifischen Wärmekapazität des Menschen die Gesamtkörperwärmemenge nach der Formel von Burton errechnet. In der statistischen Auswertung der Ergebnisse konnte zu den Zeitpunkten $30 \mathrm{~min}, 45 \mathrm{~min}$ und $60 \mathrm{~min}$ bei den Probanden der Versuchsreihe „konvenktiver Luftwärmer" eine signifikant höhere Gesamtkörperwärmemenge festgestellt werden.

\subsection{Ausblick}

Die vorliegende Probandensimulation konnte deutlich machen, dass konvektive Luftwärmer, welche im innerklinischen Bereich zur Anwendung kommen, auch unter deutlich kälteren Umgebungstemperaturen, wie sie beispielsweise unter präklinischen Bedingungen herrschen, sicher funktionieren und hoch effektiv zur Wärmung von Patienten und Vermeidung von hypothermen Zuständen sind. Auch konnte gezeigt werden, dass diese Geräte der passiven Wärmemethode mittels Vlies-Einmaldecke deutlich überlegen sind. Die vorliegende Studie konnte dabei belegen, dass dieser Einsatz sicher erfolgen kann.

Durch den Einsatz einer Vlies-Einmaldecke kann der Wärmeverlust zumindest bei gesunden Probanden aufgehalten werden.

Aufgrund der Tatsache, dass die Hypothermie insbesondere nach Traumata einen erheblichen Einfluss auf den weiteren Verlauf des Patienten hat und die Vermeidung und Bekämpfung von hypothermen Zuständen einen hohen Stellenwert hat, ist, als Ergebnis dieser Arbeit, der Einsatz von konvektiven Luftwärmern bereits unter präklinischen Bedingungen zu fordern und zu etablieren. 
Diskussion

Sollte an solchen Einsatzorten kein konvektiver Luftwärmer vorhanden sein, sollte zumindest eine passive Wärmemethode, wie eine Vlies-Einmaldecke, konsequent zur Anwendung kommen. 


\section{Zusammenfassung}

Hypotherme Zustände in Zusammenhang mit unfallbedingten schweren Verletzungen sind häufig und haben weitreichende Folgen auf die Hämostase und Immunologie (Tsuei und Kearney 2004). Die Maßnahmen, die akzidentelle Hypothermie zu bekämpfen oder ihre Entstehung zu verhindern, müssen daher möglichst früh, also in der prä-hospitalen Phase begonnen werden. Maßnahmen, die aktuell in der Präklinik an Unfallstellen zur Anwendung kommen, sind jedoch zum überwiegenden Teil passive Wärmemethoden, wie Decken o.ä. Die im innerklinischen Bereich für die Wärmung von Patienten etablierten konvektiven Luftwärmer kommen bisher frühestens in der Schockraumphase zur Anwendung.

Für die Frage, ob die Wärmung mittels Decken oder konvektiven Luftwärmern im präklinischen Bereich unter deutlich niedrigeren Umgebungstemperaturen funktioniert, gibt es bisher nur eine Studie. Diese wurde jedoch mit Geräten durchgeführt, welche aufgrund der abgegebenen Temperaturen für den Einsatz am Patienten nicht sicher geeignet sind (Jebens 2014). Diese Frage und ob das eine Verfahren dem anderen in der Effektivität der Erwärmung überlegen ist, sollte mit dieser Studie beantwortet werden.

Hierzu durchliefen 32 gesunde Probanden randomisiert an unterschiedlichen Tagen mit einer Pause von mindestens $24 \mathrm{~h}$ zwei Versuchsreihen. Sie wurden jeweils für 21 Minuten bekleidet mit Unterwäsche, T-Shirt, Shorts und Socken bei einer Umgebungstemperatur von $3{ }^{\circ} \mathrm{C}$ auf einem Stuhl platziert. Nach Ablauf dieser Zeit wurden sie entweder für weitere 39 Minuten mit einer Vlies-Einmaldecke, wie sie standardisiert bei Einsätzen von Feuerwehren und Rettungsdiensten zur Anwendung kommt, gewärmt oder ein konvektiver Luftwärmer, wie er im innerklinischen Bereich standardisiert verwendet wird, wurde mit einer entsprechenden Wärmedecke für die weiteren 39 Minuten eingesetzt.

Als Messwerte wurden dreiminütlich neben den Vitalparametern Blutdruck, Herzfrequenz und periphere Sauerstoffsättigung die Körpertemperatur mittels tympanaler Temperaturmessung und vier weiterer Hauttemperaturmesspunkte ermittelt. In der statistischen Auswertung konnte gezeigt werden, dass der konvektive Luftwärmer deutlich effektiver ist als der Einsatz der Vlies-Einmaldecke. Beide Verfahren können hierbei sicher unter präklinischen Bedingungen zum Einsatz kommen. Als Ergebnis dieser Arbeit ist der Einsatz von konvektiven Luftwärmern bereits in der prä-hospitalen Phase an der Unfallstelle zu fordern und zu etablieren. Auch sollte das Rettungsdienstpersonal gerade bei schwer 
Zusammenfassung

verletzten Patienten noch mehr auf ein effektives Temperaturmanagement achten und zumindest sollte eine Vlies-Einmaldecke oder etwas ähnliches Verwendung finden, um einem weiteren Wärmeverlust vorzubeugen. Die Überwachung der Körpertemperatur muss zum Standardmonitoring gehören. 


\section{Anhang}

\subsection{Probandenaufklärungsbogen}

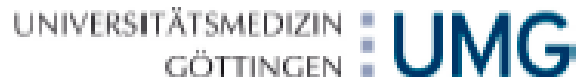

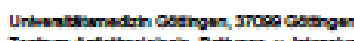

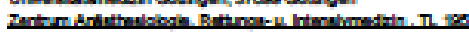

Herrl Frau

Probandin
Zontrum AnJetheclologlo, Rottunoc- und Intenclvmedizln Geschatufohrender Lehter Direktor Ansatheslologle

Prof. Dr. med. Michael Quintel

Projekttelter

$$
\text { Dr. med. Thm Heyne }
$$

Robert-Koch-8tr. 40, 37075 Gottingen Adroces

0551/39-8825 Tolofon

0551/ 39-8576 Fax

Itm.hemeGmedizin unt-ooettingende E-Mall muw.zarimed unt-oceltingen. de Homepago

6. Oktober 2015

Klinische Studie zur Überprüfung "Präklinischer Behandlungsoptionen der akzidentellen Hypothermie"

\section{Auflklärungs-/ Informationsschrift für Probanden}

Sehr geehrte Probandin, sehr geehrter Proband,

wir möchten Sie herzlich einladen an einer Untersuchung zur Überprüfung verschiedener Methoden zur Bekämpfung. bzw. Vermeidung einer Unterkühlung (Hypothermie) teilzunehmen.

Die Teilhahme an dieser Studie ist freiwiliig und auch nach Einwilligung kann diese von lhnen jederzeit widerrufen werden.

Um Ihnen einen Überblick über die Hintergründe und den Ablauf dieser Untersuchung zu verschaffen, haben wir für Sie dieses Informationsblatt entworfen und möchten Sie bitten, dieses aufmerksam durchzulesen. Sollten Sie weitere Fragen zu dieser Studie haben, beantworten lhnen die Studienärate lhre Fragen gerne.

\section{Hintergrund dieser Studie}

In der Bundesrepublik Deutschland ist der häufigste Grund für junge Menschen unter 45 Jahren zu versterben, der Unfalltod. Kommt es zu einem Unfall, wo ein Mensch eine potentiell tödliche Verletzung erlitten hat, erhöht eine Unterkühlung, d.h. ein Absinken der Körpertemperatur unter den Normalwert, die Wahrscheinlichkeit an dieser Verletzung zu versterben deutlich! Der Grund dafür liegt u.a. an der Beeinflussung der körpereigenen Blutgerinnungsfunktion durch die verminderte Körpertemperatur. 


\section{UNIVERSITÄTSMEDIZIN $: \mathbf{M O}$
GÖTTINGEN $: \mathbf{O}$}

Damit ist die Bekämpfung, oder Vermeidung eines Absinkens der Körpertemperatur eines schwerverletzten Patienten von groler Bedeutung, um das Überleben von Trauma-Patienten zukünftig verbessern zu können.

\section{Ziel der Untersuchung}

Mit dieser Studie soll untersucht werden, ob es gelingt bereits in der präklinischen Behandlung, d.h. b während der Behandlung durch den Rettungsdienst, mit unterschiedlichen Methoden eine Stabilisierung bzw. Anstieg der Körperkerntemperatur, z.B. von in Ihren Fahrzeugen eingeklemmten Verkehrsunfallopfern zu erreichen und damit eine Auskühlung zu verhindern. Im Rahmen dieser Untersuchung sollen die Methoden der Erwärmung mit einem Warmluftgebläse im Vergleich zu der Wirkung einer Vlies-Patientendecke auf lhre Effektivität überprüft werden.

\section{Ablauf der Studie}

Die Studie besteht aus zwei Untersuchungsabschnitten von jeweils einer Stunde Dauer. Dies bedeutet für Sie, dass Sie an zwei unterschiedlichen Tagen im Abstand von mindestens 24 Stunden, mit jeweils der einen, oder anderen Methode gewärmt werden.

Der Ablauf jedes Versuchsdurchgangs ist exalt gleich und Iauft folgendermaßen ab: Nach Abholung durch das Untersucherteam von lhnen zu Hause und dem Transport in ein Kühlhaus in der Nähe von Hamburg, werden sie mit einer standardisierten Bekleidung, welche die zerrissenen Kleidungsstücke eines Unfallpatienten demonstrieren soll, (Sandalen, Unterwäsche, T-Shirt und Shorts beschränkt) versorgt und an einen Überwachungsmonitor angeschlossen. Dieser überwacht kontinuierlich Ihre Körperfunktionen, bestehend aus EKG, Pulsoxymetrie, Blutdruck und der Körpertemperatur, welche über eine tympanale Sonde (Messsonde im Gehörgang eines Ohres) gemessen wird. Dann nehmen Sie auf einem Stuhl, welcher in dem Kühlhaus bei einer Umgebungstemperatur von ca. $+2^{\circ} \mathrm{C}$ platziert ist, Platz.

Beim ersten Versuchsdurchgang ziehen Sie einen Umschlag in welchem Sie die Erwärmungsmethode (VliesEinmalpatientendecke, oder Warmluftgebläse) auslosen, welche bei diesem Versuch angewendet werden soll und teilen die im Umschlag enthaltene Methode dem Untersuchungsteam mit.

Dann beginnt bereits die Messung, d.h. Ihre überwachten Körperfunktionen werden von dem Studienteamkontinuierlich überwacht und in entsprechenden Protokollen dokumentiert. Für einen Zeitraum von 20 Minuten erfolgt keine altive Erwärmung. Dieser Zeitraum simuliert den normalen Ablauf vom Unfallzeitpunkt bis zum Beginn der Rettungsmaßnahmen durch Feuerwehr und Rettungsdienst.

Ab der 21. Minute wird dann mit der aktiven Erwärmung begonnen, d.h. im ersten Messzyklus werden Sie über einen Zeitraum von $\mathbf{4 0}$ Minuten mit der von lhnen in dem Umochlag gezogenen Methode gewärmt:

Methode A Vies-Einmalpatientendecke

Wirmung mittels einer Vies-Einmalpatientendecke

bzw.

Methode B Warmluftgeblëse

Nach Überlegen Wärmedecke wird mittels Warmluftgebläses auf $43^{\circ}$ Celsius erwärmte luft in die Decke eingeleitet

Nach Abschluss der Messung werden Sie in einem gewärmten Raum gebracht, dort mit warmer Kleidung sowie Heiß-, oder Kaltgetränken versorgt und über einen Zeitraum von mindestens 2 Stunden durch die Studienärate überwacht. 
Nach Ablauf dieser Zeit werden Sie, wenn Sie sich wohlfühlen, durch einen Fahrdienst nach Hause gebracht, wo wir Sie in die Obhut einer Betreuungsperson geben. Bitte stellen Sie sicher, dass bei lhrer Rückkehr eine Ihnen vertraute Person Sie bis zum Folgetag zur Seite steht.

Nach Ablauf eines Zeitraumes von mindestens 24 Stunden nach Durchführung der ersten Messreihe, erfolgt dann die Durchführung des zweiten Untersuchungagangs, allerdings fallt dann die Wahl der Warmemethode weE, da bei jedem Probanden beide Methoden überprüft werden sollen.

\section{Welche Gefahren bestehen für Sie?}

Aufgrund zahlreicher ähnlicher Untersuchungen im klinischen Bereich sind schwerwiegende gesundheitliche folgen für Sie nicht zu erwarten! Ausschlielen können wir nicht, dass Sie im Rahmen dieser Studie an einem Infekte der oberen Atemwege, Fieber, einer Lungenentzündung, oder -im schlimmsten Fall - an Atemstörungen erkranken. Während der Untersuchung ist ein Ārzteteam mit umfangreicher Notfallausrüstung im Kühlhaus anwesend und kann bei eventuellen Notfallen umgehend qualifizierte Erstversorgung gewährleisten.

Sollte Sie sich im Anschluss an diese Untersuchung unwohl fühlen, suchen Sie umgehend Ihren Hausarzt auf und informieren Sie den Projektleiter, welchen Sie über die Kontaktdaten im Briefkopf erreichen.

\section{Datenschutz}

Alle im Rahmen dieser Studie aufgezeichneten Befunde und persönliche Informationen werden streng vertraulich behandelt. Sie werden auf Prüfprotokollen in pseudonymisierter form festgehalten und unter Wahrung des gesetzlichen Datenschutzes ausgewertet.

Die Datenspeicherseit der erhobenen Daten betrögt 10 Jahre, thre personenbesogenen Daten werden nicht an Dritte weitergegeben.

\section{Weitere Himweise}

Falls Sie bereit sind, an dieser Studie teilzunehmen, müssen Sie die Anweisungen Ihres Prüfarates genau befolgen. Wenn Sie die Behandlung beenden möchten oder unvorhergesehene Ereignisse auftreten, sollten Sie lhren Studienarat sofort benachrichtigen.

Die Untersuchung wird nach Beratung und zustimmender Bewertung durch die Ethikkommission der Universität Göttingen nach den Prinzipien des Weltärzteverbundes (Deklaration won Helsinki) durchgeführt.

Es steht lhnen wöllig frei, an dieser klinischen Studie teilzunehmen. Auch nach Einwilligung in die Studie können Sie diese Einwilligung ohne Angabe von Gründen jederzeit zurückziehen, ohne dass lhnen daraus Nachteile entstehen werden! 


\title{
6.2. Einwilligungserklärung Probanden
}

\author{
Einverständniserklärung \\ zur Teilnahme an der Studie zur Untersuchung \\ “Präklinischer Behandlungsoptionen der akzidentellen Hypothermie"
}

Ich, (Name, Vorname), habe die vorliegende Probandenaufklärung gelesen und den Inhalt verstanden. Ich hatte ausreichend Gelegenheit, weitere Einzelheiten zu der Untersuchung zu erfragen und genügend Zeit, um meine Entscheidung unbeeinflusst zu treffen.

Darüber hinaus wurde ich in einem persönlichen Gespräch mit Herrn / Frau umfassend

über die geplante Studie informiert und über meine Aufgabe sowie eventuelle Nutzen und Risiken, die im Rahmen dieser Studie für mich entstehen, aufgeklärt und weiß, dass die Anfahrt auf eigenes Risiko erfolgt.

Ich habe keine weiteren Fragen und bin belehrt worden, dass für eventuelle spātere Rückfragen mir die Studienärzte jederzeit zur Verfügung stehen.

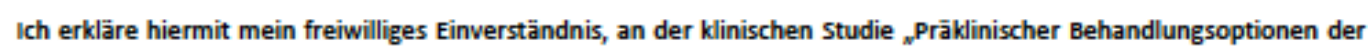
akzidentellen Hypothermie ${ }^{\alpha}$ teilzunehmen.

Mir ist bekannt, dass ich meine Einwilligung jederzeit widerrufen kann. Mein Rücktritt kann ohne Angabe von Gründen erfolgen, hieraus entstehen mit keine Nachteile. Eine Kopie dieser Einwilligungserklärung und die Probandenaufklärung habe ich erhalten.

\section{Hinweis zum Datenschutz:}

Bei dieser Studie werden die Vorschriften über die ärztliche Schweigepflicht und den Datenschutz eingehalten. Es werden thre persönlichen Daten sowie die Messergebnisse erhoben, gespeichert und pseudonymisiert, d.h. Ihr Name und die Untersuchungsergebnisse werden durch die Prüfärzte mittels eines Nummerncode verschlüsselt, so dass nur durch die verantwortlichen Studienärzte eine Zuordnung der Messergebnisse zu Ihrer Person möglich ist! Die Datenspeicherzeit der erhobenen Daten beträgt 10 Jahre, Ihre personenbezogenen Daten werden dabei nicht an Dritte weitergegeben.

Im Falle des Widerrufs Ihrer Einwilligung werden die gespeicherten Daten vernichtet. 


\section{Literaturverzeichnis}

Aitken LM, Hendrikz JK, Dulhunty JM, Rudd MJ (2009): Hypothermia and associated outcomes in seriously injured trauma patients in a predominantly sub-tropical climate. Resuscitation 80(2), 217-223

Badjatia N, Strongilis E, Gordon E, Prescutti M, Fernandez L, Fernandez A, Buitrago M, Schmidt J, Ostapkovich N, Mayer S (2008): Metabolic impact of shivering during therapeutic temperature modulation: the Bedside Shivering Assessment Scale. Stroke $\underline{39}(12), 3242-3247$

Beilman G, Blondet J, Nelson T, Nathens A, Moore F, Rhee P, Puyana J, Moore E, Cohn S (2009): Early Hypothermia in Severely Injured Trauma Patients Is a Significant Risk Factor for Multiple Organ Dysfunction Syndrome but Not Mortality. Ann. Surg. 249(5), 845-850

Bennett J, Ramachandra V, Webster J, Carli F (1994): Prevention of hypothermia during hip surgery: effect of passive compared with active skin surface warming. $\mathrm{Br} J$ Anaesth $\underline{73}(2), 180-183$

Benzinger T (1969): Heat regulation: homoeostasis of central temperature in man. Physiol Revs $\underline{49}$, 671-759

Bernabei AF, Levison MA, Bender J S (1992): The effects of hypothermia and injury severity on blood loss during trauma laparotomy. J Trauma 33(6), 835-839

Bock M, Muller J, Bach A, Bohrer H, Martin E, Motsch J (1998): Effects of preinduction and

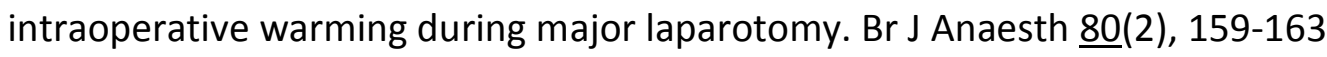

Bräuer A, Perl T, Wittkopp E, Braun U, Weyland W (2000): Stellenwert eines reflektierenden Isolationsmaterials (Thermadrape) zur Verhinderung intraoperativer Hypothermie. Anaesthesiol Intensivmed Notfallmed Schmerzther $\underline{35}$, 756-762

Bräuer A, English M, Steinmetz N (2002): Comparison of forced-air warming systems with upper body blankets using a copper manikin of the human body. Acta Anaesthesiol Scand $\underline{46}$, 965-972

Bräuer A, Perl T, Quintel M (2006): Perioperatives Wärmemanagement. Anaesthesist $\underline{55}(12)$, $1321-1340$

Brück K, Baum E, Schwennick H (1976): Cold-adaptive modifications in non induced by repeated short-term cold exposures and during a 10 day and -night cold exposure. Pflügers Arch $\underline{363}, 125-133$

Buggy D, Crossley A (2000): Thermoregulation, mild perioperative hypothermia and postanaesthetic shivering. Br J Anaesth $\underline{84}$, 615-628

Burton A (1935): The average temperature of the tissues of the body. J Nutr $\underline{9}, 264-267$ 
Christ F, Lackner C (2004): Präklinische Versorgung des Patienten mit Schock. Internist $\underline{45}$, 267-276

Copes WS, Champion HR, Sacco W J, Lawnick MM, Keast SL, Bain LW (1988): The Injury Severity Score revisited. J Trauma 28, 69-77

Crandall C, González-Alonso J (2010): Cardiovascular function in the heat-stressed human. Acta Physiol (Oxf) 199(4), 407-423

Daniel I, Sessler D (1997): Mild perioperative hypothermia. N Engl J Med 336(24), 1730-1737

Danzl D, Pozos R (1994): Accidental hypothermia. N Engl J Med 331(26), 1756-1760

Dawkins M, Scopes J (1965): Non-shivering thermogenesis and brown adipose tissue in the human new-born infant. Natur 206, 201-202

Dickenson A (1977): Specific responses of rat raphe neurones to skin temperature. J Physiol $\underline{273}, 277-293$

Flavahan N (1991): The role of vascular alpha2-adrenoceptors as cutaneous thermosensors. News Physiol Sci $\underline{6}$, 251-255

Fritz U, Bräuer A, English M (1998): Aktive Wärmetherapie. Anaesthesiol Intensivmed Notfallmed Schmerzther 33 , 389-392

Gentilello LM, Jurkovich GJ, Stark MS, Hassantash SA, O'Keefe G E (1997): Is hypothermia in the victim of major trauma protective or harmful? A randomized, prospective study. Ann Surg 226(4), 439-447; discussion 447-439

Giesbrecht GG (2000): Cold stress, near drowning and accidental hypothermia: a review. Aviat Space Environ Med $\underline{71}(7)$, 733-752

Gunga HC: Wärmehaushalt und Temperaturregulation. In: v. Speckmann E-J, Hescheler J, Köhling R (Hrsg.): Physiologie. 6. Auflage; Elsevier, Urban \& Fischer, München 2013, 603-628

Guyton AC, Hall JE: Textbook of medical physiology. 9. Auflage; W.B. Saunders, Philadelphia 1996

Henriksson O, Lundgren JP, Kuklane K, Holmer I, Bjornstig U (2009): Protection against cold in prehospital care-thermal insulation properties of blankets and rescue bags in different wind conditions. Prehosp Disaster Med 24(5), 408-415

Hervey G: Thermoregulation. In: Emslie-Smith D, Paterson C, Scratcherd T, Read N (Hrsg.): Textbook of Physiology. Churchill-Livingstone, Edinburgh 1988, 510-533 
Hess J, Lawson J (2006): The coagulopathy of trauma versus disseminated intravascular coagulation. J Trauma $\underline{60}(6), 12-19$

Hess J, Brohi K, Dutton R (2008): The coagulopathy of trauma: a review of mechanisms. J Trauma $\underline{65}, 748-754$

Heuer L: Prospektive Untersuchung zur Wirksamkeit der aktiven perioperativen Wärmetherapie auf den Verlauf der Körpertemperatur und den perioperativen Verlauf polytraumatisierter Patienten. Med. Diss. Bochum 2000

Hildebrand F, Giannoudis P, van Griensven M, Chawda M, Pape H (2004): Pathophysiologic changes and effects of hypothermia on outcome in elective surgery and trauma patients. Am J Surg 187(3), 363-371

Hildebrand F, van Griensven M, Giannoudis P (2005): Impact of hypothermia on the immunologic response after trauma and elective surgery. Surg Technol Int $\underline{14}$, 41-50

Hildebrand F, Probst C, Frink M, Huber-Wagner S, Krettek C (2009): Bedeutung der Hypothermie beim Polytrauma. Unfallchirurg 112, 959-964

Hynson JM, Sessler DI (1992): Intraoperative warming therapies: a comparison of three devices. J Clin Anesth 4 (3), 194-199

Hynson J, Sessler D, Moayeri A, McGuire J, Schroeder M (1993): The effects of preinduction warming on temperature and blood pressure during propofol/nitrous oxide anesthesia. Anesthesiology $\underline{79}, 219-228$

Inaba K, Teixeira PG, Rhee P, Brown C, Salim A, DuBose J, Chan LS, Demetriades D (2009): Mortality impact of hypothermia after cavitary explorations in trauma. World J Surg $\underline{33}(4), 864-869$

Ireland S, Endacott R, Cameron P, Fitzgerald M, Paul E (2011): The incidence and significance of accidental hypothermia in major trauma - A prospective observational study. Resuscitation 82(3), 300-306

Jebens C: Vergleich präklinischer Behandlungsoptionen der akzidentellen Hypo-thermie nach Einklemmungstrauma - Eine Probandensimulation. Med. Diss. Göttingen 2014

Jirak Z, Jokl M, Stverák J, Pechlát R, Coufalov H (1975): Correction factors in skin temperature measurement. J Appl Physiol 38(4), 752-756

Johnston T, Chen Y, Reed R (1994): Functional equivalence of hypothermia to specific clotting factor deficiencies. J Trauma 37, 413-417

Jurkovich G, Greiser W, Luterman A, Curreri P (1987): Hypothermia in trauma victims: an ominous predictor of survival. J Trauma 27, 1919-1924 
Just B, Delva E, Camus Y, Lienhart A (1992): Oxygen uptake during recovery following naloxone: relationship with intraoperative heat loss. Anesthesiology $\underline{76}, 60-64$

Kermode J, Zheng Q, Milner E (1999): Marked temperature dependence of the platelet calcium signal induced by human von Willebrand factor. Blood 94, 199-207

Kheirbek T, Kochanek AR, Alam HB (2009): Hypothermia in bleeding trauma: a friend or a foe? Scand J Trauma Resusc Emerg Med 17, 65

Kobbe P, Lichte P, Wellmann M, Hildebrand F, Nast-Kolb D, Waydhas C, Oberbeck R (2009): Bedeutung der Hypothermie in der Traumatologie. Unfallchirurg 112(12), 1055-1061

Kreimeier U, Dirks B, Wenzel V (2008): Evidenzbasierte Notfallmedizin: Perspektiven. Notfall Rettungsmed 11, 18-24

Langhelle A, Lockey D, Harris T, Davies G (2012): Body temperature of trauma patients on admission to hospital: a comparison of anaesthetised and non-anaesthetised patients. Emerg Med J 29 (3), 239-242

Lier H (2008): Hypothermie und die tödliche Triade. Notfall Rettungsmed 11, 377-380

Lier H, Kampe S, Schroder S (2007): Rahmenbedingungen für eine intakte Hämostase. Anaesthesist $\underline{56}, 239-251$

Lier H, Krep H, Schroeder S, Stuber F (2008): Preconditions of hemostasis in trauma: a review. The influence of acidosis, hypocalcemia, anemia, and hypothermia on functional hemostasis in trauma. J Trauma $\underline{65}$ (4), 951-960

Luxem L, Schlechtriemen, T, Kremer M, Gerdts KG: Traumatologische Notfälle. In: Kühn D, Luxem J, Runggaldier K: Rettungsdienst. 5. Auflage; Urban \& Schwarzenberg, München 2010, 435-482

Manson J, Thiemermann C, Brohi K (2012): Trauma alarmins as activators of damage-induced inflammation. Br J Surg 99 Suppl 1, 12-20

Martin R, Kilgo P, Miller P, Hoth J, Meredith J, Chang M (2005): Injury associated hypothermia: an analysis of the 2004 National Trauma Data Bank. Shock 24, 114-118

Martini WZ (2009): Fibrinogen metabolic responses to trauma. Scand J Trauma Resusc Emerg Med 17,2

Mccullough L, Arora S (2004): Diagnosis and Treatment of Hypothermia. Am Fam Physician 70(12), 2325-2332

Mendlowitz M (1948): The specific heat of human blood. Science 107, 97-98

Mitra B, Tullio F, Cameron PA, Fitzgerald M (2012): Trauma patients with the 'triad of death'. Emerg Med J $\underline{29}(8), 622-625$ 
Mommsen P, Zeckey C, Frink M, Krettek C, Hildebrand F (2012): Accidental hypothermia in multiple trauma patients. Zentralbl Chir 137(3), 264-269

Mommsen $\mathrm{P}$, Andruszkow $\mathrm{H}$, Fromke $\mathrm{C}$, Zeckey $\mathrm{C}$, Wagner $\mathrm{U}$, van Griensven $\mathrm{M}$, Frink $\mathrm{M}$, Krettek C, Hildebrand F (2013): Effects of accidental hypothermia on posttraumatic complications and outcome in multiple trauma patients. Injury $\underline{44}(1), 86-90$

NAEMT (Hrsg.sp): Präklinisches Traumamanagement: Das PHTLS-Konzept. Elsevier, München 2009, 406-407

Park KH, Lee KH, Kim H (2013): Effect of hypothermia on coagulatory function and survival in Sprague-Dawley rats exposed to uncontrolled haemorrhagic shock. Injury $\underline{44}(1), 91-96$

Persson PB: Energie- und Wärmehaushalt, Thermoregulation. In: Schmidt R, Lang F, Heckmann M (Hrsg.): Physiologie des Menschen. Springer, Berlin Heidelberg 2011, 883-898

Pierau F, Wurster R (1981): Primary afferent input from cutaneous thermoreceptors. Fed Proc 40, 2819-2824

Poulos D (1981): Central processing of cutaneous tmeperature information. Fed Proc $\underline{40}$, 2825-2829

Ramanathan N (1964): A new weighting system for mean surface temperature of the humanbody. J Appl Physiol 19, 531-533

Riskin D, Tsai T, Riskin L (2009): Massive transfusion protocols: the role of aggressive resuscitation versus product ration in mortality reduction. J Am Coll Surg 209, 198-205

Rutherford EJ, Fusco MA, Nunn CR, Bass JG, Eddy VA, Morris JA, Jr. (1998): Hypothermia in critically ill trauma patients. Injury $\underline{29}(8), 605-608$

Scherer R (1997): Intraoperative Wärmekonservierung - Viel Lärm um heiße Luft? Anaesthesist $\underline{46}, 81-90$

Seamon MJ, Wobb J, Gaughan J P, Kulp H, Kamel I, Dempsey DT (2012): The effects of intraoperative hypothermia on surgical site infection: an analysis of 524 trauma laparotomies. Ann Surg 255(4), 789-795

Seekamp A, Ziegler M, Biank J, Grotz G (1996): Die Bedeutung der Hypothermie beim polytraumatisierten Patienten. Unfallchirurg $\underline{99}(2), 100-105$

Seekamp A, van Griensven M, Hildebrandt F, Wahlers T, Tscherne H (1999): AdenosineTriphosphate in Trauma-Related and Elective Hypothermia. J Trauma 477(4), 673-683

Seibt W: Physik für Mediziner. 6. Auflage; Thieme, Stuttgart 2009, 159-167

Sessler D: Temperature Monitoring. Churchill Livingstone, New-York 1994 
Sessler DI, Moayeri A (1990): Skin-surface warming: heat flux and central temperature. Anesthesiology $\underline{73}(2), 218-224$

Shafi S, Elliott A, Gentilello L (2005): Is hypothermia simply a marker of shock and injury severity or an independent risk factor for mortality in trauma patients? Analysis of a large national trauma registry. J Trauma $\underline{5}, 1081-1085$

Steinemann S, Shackford SR, Davis JW (1990): Implications of admission hypothermia in trauma patients. J Trauma $\underline{30}(2), 200-202$

Sterba JA (1991): Efficacy and safety of prehospital rewarming techniques to treat accidental hypothermia. Ann Emerg Med 20(8), 896-901

Südmersen J, Heyne H: Technische Hilfeleistung bei PKW-Unfällen. In: Cimolino U: Einsatzpraxis. 2. Auflage; Ecomed-Verlag, Landsberg 2008, 212-214

Sundberg J, Estrada C, Jenkins C, Ray J, Abramo T (2011): Hypothermia is associated with poor outcome in pediatric trauma patients. Am J Emerg Med 29(9), 1019-1022

Thomassen O, Faerevik H, Osteras O, Sunde GA, Zakariassen E, Sandsund M, Heltne JK, Brattebo G (2011): Comparison of three different prehospital wrapping methods for preventing hypothermia--a crossover study in humans. Scand J Trauma Resusc Emerg Med 19, 41

Trentzsch H, Huber-Wagner S, Hildebrand F, Kanz K, Faist E, Piltz S, Lefering R (2012): Hypothermia for Prediction of Death in Severely Injured Blunt Trauma Patients. Shock $\underline{37}(2), 131-139$

Tritthart H: Physik und Biophysik für Mediziner. Schattauer, Stuttgart 2011, 218-222

Tsuei BJ, Kearney PA (2004): Hypothermia in the trauma patient. Injury $\underline{35}$ (1), 7-15

von Hintzenstern U, Saefkow M, Mörer O: Infusionsmaterial; in: i.v. Infusion, Transfusion, Parenterale Ernährung; hrsg. v. von Hintzenstern U. 3. Auflage; Urban \& Fischer, München 2004, 17-62

Wade CE, Salinas J, Eastridge BJ, McManus JG, Holcomb JB (2011): Admission hypo- or hyperthermia and survival after trauma in civilian and military environments. Int $\mathrm{J}$ Emerg Med $\underline{4}(1), 35$

Wang HE, Callaway CW, Peitzman AB, Tisherman SA (2005): Admission hypothermia and outcome after major trauma. Critical Care Medicine 33(6), 1296-1301

Weuster M, Bruck A, Lippross S, Menzdorf L, Fitschen-Oestern S, Behrendt P, Iden T, Hocker J, Lefering R, Seekamp A, et al. (2016): Epidemiology of accidental hypothermia in polytrauma patients: An analysis of 15,230 patients of the TraumaRegister DGU. J Trauma Acute Care Surg 81(5), 905-912 
Wyss C, Brengelmann G, Johnson J, Rowell L, Silverstein D (1975): Altered control of skin blood flow at high skin and core temperature. J Appl Physiol 38, 839-845

Yenari M, Palmer J, Bracci P (1995): Thrombolysis with tissue plasminogen activator (tPA) is temperature dependent. Thromb Res $\underline{77}$, 475-481

Zink W, Bernhard M, Keul W, Martin E, Volkl A, Gries A (2004): [Invasive techniques in emergency medicine. I. Practice-oriented training concept to ensure adequately qualified emergency physicians]. Anaesthesist $\underline{53}(11), 1086-1092$

\subsection{Internetquellen}

http://www.traumaregister-dgu.de/fileadmin/user_upload/traumaregisterdgu.de/docs/Downloads/TR-DGU-Jahresbericht_2015.pdf. Zugriff am 15.01.2017

http://reintex.com/Einwegdecken/index.html. Zugriff am 16.07.2015

https://www.destatis.de/DE/PresseService/Presse/Pressekonferenzen/2016/Unfallentwicklu ng_2015/Pressebroschuere_unfallentwicklung.pdf?_blob=publicationFile. Zugriff am 07.06.2017

http://www.rki.de/DE/Content/Gesundheitsmonitoring/Gesundheitsberichterstattung/GBE DownloadsB/unfallbericht_geda.pdf\%3F__blob\%3DpublicationFile.

Zugriff am 05.10.2015

https://de.wikipedia.org/wiki/Zeitreihe_der_Lufttemperatur_in_Deutschland. Zugriff am 06.10.2015 


\section{DANKSAGUNG}

Bedanken möchte ich mich bei Herrn Prof. Anselm Bräuer für dieses interessante und für die präklinische Phase so wichtige Dissertationsthema.

Mein Dank geht im Besonderen an Herrn Dr. Tim Heyne, der sich die Zeit genommen hat, mich so außergewöhnlich gut und ideenreich zu unterstützen und betreuen.

Des Weiteren danke ich meinem allerbesten Freund Dr.Christopher Jebens, der mir immer mit Rat und Tat zur Seite gestanden und über mache schwache Stunde geholfen hat.

Ich danke meinen Eltern ganz herzlich, die zur geistigen und kulinarischen Versorgung der Probanden und mir, in den „kalten Zeiten“, zur Seite standen.

Ein besonderer Dank geht an meine Freunde und Bekannte, die sich freiwillig der Kälte ausgesetzt und für diese Studie zur Verfügung gestellt haben. 
Lebenslauf

LEBENSLAUF 